\title{
Quasiperiodic spin-orbit motion and spin tunes in storage rings
}

\author{
D. P. Barber, ${ }^{1}$ J. A. Ellison, ${ }^{2}$ and K. Heinemann ${ }^{1}$ \\ ${ }^{1}$ Deutsches Elektronen-Synchrotron (DESY), 22603 Hamburg, Germany \\ ${ }^{2}$ Department of Mathematics and Statistics, University of New Mexico, Albuquerque, New Mexico 87131, USA
}

(Received 30 November 2003; published 17 December 2004)

\begin{abstract}
We present an in-depth analysis of the concept of spin precession frequency for integrable orbital motion in storage rings. Spin motion on the periodic closed orbit of a storage ring can be analyzed in terms of the Floquet theorem for equations of motion with periodic parameters, and a spin precession frequency emerges in a Floquet exponent as an additional frequency of the system. To define a spin precession frequency on nonperiodic synchrobetatron orbits we exploit the important concept of quasiperiodicity. This allows a generalization of the Floquet theorem so that a spin precession frequency can be defined in this case, too. This frequency appears in a Floquet-like exponent as an additional frequency in the system in analogy with the case of motion on the closed orbit. These circumstances lead naturally to the definition of the uniform precession rate and a definition of spin tune. A spin tune is a uniform precession rate obtained when certain conditions are fulfilled. Having defined spin tune we define spin-orbit resonance on synchrobetatron orbits and examine its consequences. We give conditions for the existence of uniform precession rates and spin tunes (e.g., where small divisors are controlled by applying a Diophantine condition) and illustrate the various aspects of our description with several examples. The formalism also suggests the use of spectral analysis to "measure" spin tune during computer simulations of spin motion on synchrobetatron orbits.
\end{abstract}

\section{INTRODUCTION}

This paper provides a rigorous discussion of the concept of spin precession frequency on synchrobetatron orbits in storage rings. To set the scene we begin by introducing some key physical ideas via the equations of orbit and spin motion and the notion of spin-orbit equilibrium.

The spin expectation value $\mathbf{S}$ ("the spin") in the rest frame of, for example, a proton, an electron, or a muon moving in electric and magnetic fields under the influence of the Lorentz force precesses according to the ThomasBargmann-Michel-Telegdi (T-BMT) equation [1]

$$
d \mathbf{S} / d t=\tilde{\mathbf{\Omega}}(\mathbf{E}, \mathbf{B}, \mathbf{v}) \times \mathbf{S},
$$

where the precession vector $\tilde{\boldsymbol{\Omega}}$ depends on $\mathbf{E}, \mathbf{B}$, and $\mathbf{v}$ which are respectively the electric and magnetic fields and the velocity. Particle motion with respect to the synchronous closed, i.e., periodic, orbit is described in terms of three pairs of canonical variables which we combine into a vector $u$ with six components. For example, two of the pairs can describe transverse motion and one pair can describe longitudinal (synchrotron) motion within a bunch. Since we are dealing with storage rings we take the orbital motion to be bounded. In this paper we ignore radiation, interparticle interactions, and interactions with the vacuum system. In (1.1) the independent variable is the time $t$. However, since the electric and magnetic guide fields in particle accelerators and storage rings are fixed in space it is more convenient to adopt the standard practice of replacing $t$ with the angular position around the ring, the azimuth $\theta=2 \pi s / L$, where $s$ is the distance around the ring and $L$ is the circumference.

Since $\mathbf{E}, \mathbf{B}$, and $\mathbf{v}$ depend on $u$ and $\theta$ we can now rewrite (1.1) in the form

$$
d \mathbf{S} / d \theta=\mathbf{\Omega}(\theta, u(\theta)) \times \mathbf{S},
$$

where $\boldsymbol{\Omega}$ is the precession vector obtained from $\tilde{\boldsymbol{\Omega}}$ by rescaling with $d t / d \theta$ and transforming to machine coordinates [2].

If the beam is in equilibrium, i.e., if the phase space density $\rho(\theta, u)$ is $2 \pi$-periodic in $\theta$, we then write it as $\rho_{\text {eq }}$ so that $\rho_{\text {eq }}(\theta, u)=\rho_{\text {eq }}(\theta+2 \pi, u)$. We normalize it to unity: $\int d u \rho_{\mathrm{eq}}=1$. The necessary condition for this kind of equilibrium, namely that at a fixed $u$ the fields are $2 \pi$-periodic in $\theta$, is automatically fulfilled in a storage ring. But of course the boundedness of the motion is also required. Conditions necessary for beam equilibrium and a way of calculating $\rho_{\mathrm{eq}}$ using ergodic theory and the concept of "stroboscopic averaging" are described in detail in [3].

The statistical properties of the spins are encoded in the quantum mechanical spin density matrix. But for spin $1 / 2$ particles this can be completely parametrized by the polarization vector [4]. For particle beams we need the local polarization $\mathbf{P}_{\mathrm{loc}}(\theta, u)$ at each point in phase space $u$. $\mathbf{P}_{\mathrm{loc}}(\theta, u)$ is the average of the normalized spin vectors, $\mathbf{S} /|\mathbf{S}|$, at $u$, where $|\cdot|$ denotes the Euclidean norm. We define the polarization of the whole beam, the "beam polarization," at a given azimuth as $\int d u \rho_{\mathrm{eq}} \mathbf{P}_{\text {loc }}$.

Since the T-BMT equation (1.2) is linear in $\mathbf{S}$ and since the particles at $(\theta, u)$ all see the same $\boldsymbol{\Omega}(\theta, u)$, 
$\mathbf{P}_{\text {loc }}(\theta, u(\theta))$ also obeys the T-BMT equation [5]. Furthermore, the length of $\mathbf{P}_{\text {loc }}(\theta, u)$ is constant along a phase space trajectory. For a storage ring at fixed energy, $\boldsymbol{\Omega}$ is $2 \pi$-periodic in $\theta$ at a fixed position in phase space $u$ so that $\boldsymbol{\Omega}(\theta, u)=\boldsymbol{\Omega}(\theta+2 \pi, u)$. This opens up the possibility of a spin distribution that is the same from turn to turn, i.e., in equilibrium. Then $\mathbf{P}_{\text {loc }}(\theta, u(\theta))$ not only obeys the T-BMT equation, but $\mathbf{P}_{\mathrm{loc}}(\theta, u)$ is $2 \pi$-periodic in $\theta$ for fixed $u$ and we then write it as $\mathbf{P}_{\text {eq }}$ so that $\mathbf{P}_{\mathrm{eq}}(\theta, u)=\mathbf{P}_{\mathrm{eq}}(\theta+2 \pi, u)$. We denote the unit vector along $\mathbf{P}_{\mathrm{eq}}(\theta, u)$ by $\mathbf{n}(\theta, u)$. This also obeys the T-BMT equation along orbits and is $2 \pi$-periodic in $\theta$ : $\mathbf{n}(\theta, u)=\mathbf{n}(\theta+2 \pi, u)$.

The method described in [3] for constructing $\rho_{\text {eq }}$ can be extended as in [6] for constructing $\mathbf{n}$, and from the treatments in [3] it is clear that the existence of $\rho_{\text {eq }}$ and $\mathbf{n}$ do not require that the orbital motion be integrable. But, of course, the conclusions of [3] are still valid if the motion is integrable. Moreover, particle motion in storage rings is usually close enough to integrability to allow the motion to be characterized in terms of well defined betatron and synchrotron frequencies. This, in turn, allows predictions to be made about beam stability via the concept of orbital resonance. Thus, in the remainder of this paper we will assume that the orbital motion is integrable. Then, as we shall see, the stability of spin motion can also be discussed in terms of resonance, namely, "spin-orbit resonance." Of course, integrable orbital motion and spin-orbit equilibrium are idealizations. Nevertheless, these idealizations often provide useful starting points for calculations.

For integrable particle motion the position of a particle in phase space is represented by three pairs of actionangle variables $\left(J_{i}, \phi_{i}, i=1,2,3\right)$ and is determined by a Hamiltonian $H(J)$. Thus the orbital phase space is partitioned into disjoint tori, each of which is characterized by a unique set of $J$ 's. We now define $u:=$ $\left(\phi_{1}, \phi_{2}, \phi_{3}, J_{1}, J_{2}, J_{3}\right) \equiv(\phi, J)$. The actions are constants of the motion and for fixed $J$ the constant rate of advance of each $\phi_{i}, \omega_{i}(J):=\partial H / \partial J_{i}=d \phi_{i} / d \theta$, is called an orbital tune. These frequencies are the number of oscillations per turn around the ring. In beam physics such frequencies are often referred to as tunes and we have adopted that usage. We will only consider storage rings running at fixed nominal energy.

For integrable motion, the $2 \pi$-periodicity in $\theta$ of $\boldsymbol{\Omega}$, $\mathbf{P}_{\text {eq }}$, and $\mathbf{n}$ is accompanied by $2 \pi$-periodicity in $\phi_{1}, \phi_{2}$, and $\phi_{3}$. So as well as being a solution to the T-BMT equation along orbits $u(\theta)$, $\mathbf{n}$ satisfies nontrivial periodicity conditions. In our later discussions on quasiperiodicity we will require that it also depend sufficiently regularly on the azimuth and the orbital angles, i.e., $\mathbf{n}$ must be "smooth" in the sense defined in the main text. This corresponds to the expectation that $\mathbf{P}_{\text {eq }}$ also be smooth. The equilibrium density $\rho_{\text {eq }}$ is also $2 \pi$-periodic in $\theta$, and off orbital resonance it just depends on $J$.
Since for every $J$ the field $\mathbf{n}(\theta, \phi, J)$ is invariant from turn to turn, it is now often called the invariant spin field (ISF). The ISF is a central object in the theory of polarization in storage rings [7-10]. For example, for an ISF sufficiently regular in $J$, off orbital resonance and away from the spin-orbit resonances to be defined below, an upper limit to the equilibrium beam polarization at a particular $\theta$ is $\int d J \rho_{\mathrm{eq}}(J)\left|\int d \phi \mathbf{n}(\theta, \phi, J)\right|$ and it is reached only when the $\int d \phi \mathbf{n}$ are parallel. This is easy to see by noting that if $\left|\mathbf{P}_{\mathrm{eq}}(\theta, \phi, J)\right|$ were to vary over a torus, the beam polarization would vary from turn to turn. So equilibrium implies that $\left|\mathbf{P}_{\mathrm{eq}}(\theta, \phi, J)\right|$ is constant over a torus. The maximum equilibrium polarization on each torus is reached when $\left|\mathbf{P}_{\mathrm{eq}}(\theta, \phi, J)\right|=1$. Note that a zero value for $\int d J \rho_{\text {eq }}(J)\left|\int d \phi \mathbf{n}\right|$ at some $\theta$ does not mean that the beam is depolarized. It could well be that the beam is fully polarized at each point in phase space but that the geometry of $\mathbf{n}$ causes the integral to vanish. Then, if an adiabatic change of parameters were to change the geometry of $\mathbf{n}$ so that the integral were to become nonzero, the beam polarization would reappear. Furthermore, the fact that the integral vanishes at one position $\theta$ does not mean that it vanishes at other positions. We prefer to reserve the term "depolarization" for a definitely irreversible loss of polarization such as occurs in the presence of noise or for an effectively irreversible loss of the kind that can occur during resonance crossing [11]. Although we have introduced $\mathbf{n}$ via the notion of equilibrium, the integral $\int d J \rho_{\text {eq }}(J)\left|\int d \phi \mathbf{n}\right|$ also contains useful information when the spin distribution is not in equilibrium: $\int d J \rho_{\text {eq }}(J)\left|\int d \phi \mathbf{n}\right|$ gives an upper limit for the time averaged polarization away from spin-orbit resonances. The maximum is reached on each torus when the polarization is in equilibrium and with $\left|P_{\text {eq }}(\theta, \phi, J)\right|=1$ (see [12], Sec. 2.2.8, and [13], Sec. 4.4). An example of the origin and behavior of nonequilibrium polarization is given in Fig. 9 in [6] where large oscillations are evident. However, polarimeters and particle detectors cannot collect data quickly enough to make such oscillations observable. Instead, only the time averaged polarization can be observed or exploited. But as we have just seen, we can still estimate its maximum value. That depends only on the geometry of $\mathbf{n}$ and it is reached for each torus when the spread of $\mathbf{n}$ is minimized. The ISF also provides a perfect tool for estimating the long term effects on the beam polarization of small perturbations such as radiation [7] or electric and magnetic fields which cause nonintegrable orbital motion. In particular, one begins with a spin-orbit system which is invariant from turn to turn, i.e., with an equilibrium orbital distribution and with spins set parallel to the $\mathbf{n}(\theta, u)$. Then, since the system is initially in equilibrium, the effects of the perturbations cannot be masked by the natural, potentially large, variations of the beam polarization of the kind depicted in Fig. 9 in [6]. An ability to construct $\mathbf{n}(\theta, u)$ 
for integrable orbital motion and understand its behavior is then indispensable.

For our integrable orbital motion of electrons and protons and up to energies of a few $\mathrm{GeV}$, an approximate $\mathbf{n}$ can be calculated in a first order perturbation theory by an extension of the code SLIM [14,15], and in higher order perturbation theory by the codes, SMILE [10], FORGET-MENOT [16], and SPINLIE [17]. However, for the high magnetic fields characteristic of proton rings running at energies of hundreds of $\mathrm{GeV}$, perturbative methods are inadequate. Then the method of stroboscopic averaging as in the code SPRINT [6] should be used. This is a numerical, nonperturbative algorithm and yields high accuracy for real rings even when all modes of orbit oscillation are included simultaneously. One can also use Fourier methods as in the codes SODOM2 [18] or MILES [19]. SODOM2 has been very useful for orbital motion restricted to one plane [13]. MILES gives explicit formulas which are applicable to some simple models. So far, the only practical general way to calculate the invariant spin field is to use stroboscopic averaging.

As for any dynamical system we hope to understand more about spin motion by studying its spectrum of frequencies. Various quantities, which seem at first sight to be related to spin frequencies, can be found in the literature and we will mention some in Sec. X. But a true component of a spectrum quantifies long term behavior. Thus any definition of spin precession frequency should reflect that stipulation. The choice can be further narrowed by requiring that the spectrum give useful clues about the behavior of sets of spins, and in particular about the beam polarization. After all, the experimenters using the beams in storage rings are just interested in the polarization, not individual spins.

Experience has shown that the best choice for characterizing spin motion in storage rings is the traditional one [20-22], namely, the so-called amplitude dependent spin tune (briefly "spin tune"), which we usually denote by $\omega_{s}$. Assuming $\mathbf{n}$ exists, the spin tune measures the number of spin precessions around $\mathbf{n}(\theta, u)$, per turn around the ring, for a particle on the orbit $u(\theta)$, and it provides a way to quantify the correlation between the spin motion and the orbital motion which "drives" it, and thereby forecast a qualitative aspect of spin motion, namely, the degree of regularity of the spin motion. In particular, the spin motion can in general become very erratic when a spin tune is near a low order resonance condition

$$
\omega_{s}=m_{0}+m_{1} \omega_{1}+m_{2} \omega_{2}+m_{3} \omega_{3}=m \cdot(1, \omega),
$$

where $m$ is a vector of integers and the quantity $\left|m_{1}\right|+$ $\left|m_{2}\right|+\left|m_{3}\right|$ is usually called the order of the resonance. Correspondingly, close to spin-orbit resonance $\mathbf{n}(\theta, u)$ can become a very sensitive function of $u$. This sensitivity has immediate consequences for work with polarized beams. For example, the maximum attainable equilib- rium beam polarization of a stored high energy proton beam can be unacceptably low or the rate of depolarization, due to synchrotron radiation, of a stored electron beam can be unacceptably high [7]. Note, however, that the $\left|\int d \phi \mathbf{n}\right| /(2 \pi)^{3}$ on a torus can sometimes be small away from spin-orbit resonance and that proximity to a spin-orbit resonance, especially one of high order, does not automatically imply that the $\left|\int d \phi \mathbf{n}\right| /(2 \pi)^{3}$ on a torus is low. The resonance might be very weak. Another feature of our definition of spin frequency is that, as we shall see, it is this quantity whose spectrum one obtains in a straightforward spectral analysis of spin motion during spin-orbit tracking simulations. In other words, in an ideal world with technology which could select particles on a torus at a fixed $J$, it could be measured.

Right at the resonance condition (1.3), $\mathbf{n}$ is in general nonunique. However, as we shall see, our spin-orbit systems exhibit a tendency to avoid exact spin-orbit resonance. Since $\boldsymbol{\Omega}$ in general depends on $J$ and the particle energy on the closed orbit, $\omega_{s}$ usually varies with $J$ (hence amplitude dependent spin tune), and the particle energy on the closed orbit. We will call the latter the "beam energy." We emphasize that $\mathbf{n}$ is a field over the six dimensional phase space so that synchrotron motion is built in from the start. Thus although $\omega_{s}$ varies with the beam energy and $J_{1}, J_{2}$, and $J_{3}$, it does not change during a period of synchrotron motion. If $\mathbf{n}$ were defined on four dimensional transverse phase space and the energy oscillations due to synchrotron motion were added as an afterthought, it would not be useful for describing equilibrium polarization. Instead, we would have to characterize the beam polarization using time averages. We return to this theme in Sec. X. On the closed orbit, i.e., for $J_{1}=$ $J_{2}=J_{3}=0$, an $\mathbf{n}$ exists which is independent of $\phi$. We denote it by $\mathbf{n}_{0}$ [15].

The calculation of spin tune on the closed orbit presents no problem: it can, as we shall see, be extracted from an eigenvalue of the one-turn spin map. But the definition of spin tune for $J \neq 0$, i.e., on synchrobetatron orbits, is much more subtle. Moreover, it requires precision. Notions of spin frequency for synchrobetatron orbits appearing in the literature are often not precisely presented and some appear to possess no capacity for predicting the qualitative aspects of spin motion in storage rings.

This brings us to the purpose of this paper. This is to provide a rigorous discussion of the concept of spin precession frequency on synchrobetatron orbits and thereby consolidate a framework for systematizing and classifying spin motion in storage rings. For this we make a careful mathematical study of the consequences for spin motion of the periodicities of $\boldsymbol{\Omega}$ in $\theta, \phi_{1}, \phi_{2}$, and $\phi_{3}$, using precise definitions and carefully formulated theorems and we make use of the ISF and other concepts 
which we distill from the literature and "folklore" on spin dynamics in storage rings [6-9,12,13,16,18,20-25]. For example, we will show that under the appropriate conditions, the existence of the ISF with the above mentioned periodicities implies that the $\theta$ dependence of the general solutions of (1.2) will contain five frequencies. Four of them are the orbital tunes $\omega_{1}, \omega_{2}, \omega_{3}$ and the circulation tune $\omega_{c}=1$, i.e., the frequency associated with the $2 \pi$-periodicity in $\theta$. A fifth tune emerges which, under circumstances to be described, is a spin tune $\omega_{s}$. The general solutions will then be found to be quasiperiodic with the tunes $1, \omega_{1}, \omega_{2}, \omega_{3}, \omega_{s}$. Moreover the results obtained here can be viewed as a generalization of Floquet theory. Given the confusion surrounding definitions of spin precession frequencies, the treatment of the kind that we provide here seems to be very necessary. Our assumptions about $\boldsymbol{\Omega}$ are weak enough to cover the situations of most interest for storage rings, namely, typical integrable synchrobetatron motion. Several of our theorems assume the existence of $\mathbf{n}$ but, although we have ways to find approximate $\mathbf{n}$, the determination of complete conditions for its existence is an outstanding mathematical issue. This question can, for example, be investigated using ergodic theory [26,27] and the method of stroboscopic averaging; see [3]. Moreover, simulations indicate that approximate ISFs $d o$ exist. This means that one obtains objects which, at least approximately, behave like an ISF. Moreover in some instances approximations even lead to an $\boldsymbol{\Omega}$ for which an exact ISF can be found, e.g., as in the single resonance model; see Sec. VII.

Although we have introduced the vector $\mathbf{n}$ by studying spin-orbit equilibrium, it was first discussed by Derbenev and Kondratenko as a vehicle for constructing joint action-angle variables for spin and orbital motion from their semiclassical spin-orbit Hamiltonian [21,22]; see also $[2,7,16]$. This Hamiltonian is derived from the Dirac Hamiltonian by a Foldy-Wouthuysen transformation taken to first order in $\hbar$ [7]. In that picture the spin tune emerges as the rate of advance of a spin phase [21,22]; see also [2]. The terms at first order in $\hbar$ in the Derbenev-Kondratenko Hamiltonian are those containing spin, and these terms imply a force of the SternGerlach (S-G) type [4]. The S-G forces on trajectories appear at first order in $\hbar$, and a "backreaction" on the spin of the S-G perturbation to the orbit would involve an addition to the spin precession rate of order $\hbar$. However, in this paper the effect of S-G forces on spin and orbit motion is neglected and we just operate with the Lorentz force and the T-BMT equation and for the Lorentz force and $\boldsymbol{\Omega}$ just include, as is usual, the terms of zeroth order in $\hbar$. There are several reasons for this approach. First, it is far from clear what form the S-G forces should take. In fact, there is considerable ambiguity in the choice of the S-G forces. This is covered in detail in [28] (see also [29] and the bibliography in [28]). The second ground has to do with the size of the S-G forces. Since the S-G forces are of first order in $\hbar$, they are extremely small compared to the Lorentz forces which are of zeroth order in $\hbar .^{1}$ They are also small compared to typical spurious perturbations to trajectories like noise and collective effects. So S-G forces would not cause changes of practical significance to the results that we present. In particular, in practical situations in a storage ring there would be no significant change in the phenomenology of spin-orbit resonances even if the S-G forces were to cause tiny changes in the orbital tunes. The third ground is that it is far from clear that it makes sense to treat an essentially quantum mechanical system with a classical "over interpretation" of the influence of the S-G force on the spin. An example of an effect which is not taken into account by a naive application of classical S-G forces is given in [30]. It is suggested there that long term shifts of an orbit due to $S-G$ forces will be nullified when the spin undergoes a quantum flip and the $S-G$ force then acts in the reverse direction (see [2], p. 137, for a classical perspective on this). In summary, we believe that a too literal interpretation of the S-G-like forces in the semiclassical spin-orbit Hamiltonian could lead to manipulations and conclusions of little relevance and utility for illuminating the core phenomenology of spin motion in typical storage rings [31,32]. We believe that the first priority is to begin with just the Lorentz force and the T-BMT equation. Then, as mentioned earlier, once the equilibrium state of the system has been defined, other influences such as nonlinear fields, noise, collective effects, synchrotron radiation, and the very small S-G-like effects can be included as perturbations.

The paper is structured as follows. In Sec. II we begin by discussing some important consequences of (1.2). Here we introduce the central concept of a uniform precession frame (UPF) and the associated uniform precession rate (UPR). The UPF provides a coordinate system for spin. Then in Sec. III we give a detailed discussion of spin motion on the closed orbit where $\Omega$ is independent of $\phi$ so that the Floquet theorem applies. Sections II and III contain standard results but we present them in forms which motivate their extension in later sections. Section IV contains the definition of a quasiperiodic function and collects some properties useful for the discussion following. In particular, it defines a Diophantine condition needed for handling a problem with small

\footnotetext{
${ }^{1}$ For example, at the rms radius of the $920 \mathrm{GeV} / c$ proton beam in a quadrupole magnet in the HERA proton ring [12,13], a rough estimate for the transverse $S-G$ force from the Derbenev-Kondratenko Hamiltonian gives a S-G force which is about $10^{-12}$ of the Lorentz force on a proton. At a fixed radius this ratio is essentially independent of the beam energy. The S$\mathrm{G}$ energy at that radius is of the order of $10^{-21}$ of the kinetic energy. The S-G energy in a HERA dipole magnet is of the order of $10^{-19}$ of the kinetic energy.
} 
divisors. The key ideas are formalized in Lemmas 4.3, 4.7, and 4.8. Section V uses the concept of a UPF, quasiperiodic with orbital frequencies, to define the proper UPR, the spin tune, and spin-orbit resonance. The main theorem in Sec. V is Theorem 5.3, which allows us to define equivalence classes of spin tunes. The presentation in Secs. II, III, IV, and V is deliberately rather general and abstract. Then in Sec. VI we introduce a field called the invariant frame field (IFF) which is used to construct UPFs. There we consider the angular phase space as a whole to prove theorems about the concepts introduced in Sec. V. We also connect the abstract ideas introduced earlier to a familiar physical idea, namely, that if the orbital tune were off orbital resonance $[m \cdot(1, \omega)=0$ with the vector of integers $m=0]$, the existence of a nonunique ISF would imply that the system were on spin-orbit resonance. The main theorems in Sec. VI are Theorems 6.3-6.5. Theorem 6.3 is used in the proof of Theorem 6.5 and it is generally our main tool for showing that a torus is "well-tuned." The proof of Theorem 6.3 relies on Theorem 5.3. Some examples of the formalism for model $\boldsymbol{\Omega}$ 's are presented in Secs. VI, VII, and VIII. Note that, except for some examples, we allow the number of action-angle pairs $d$ to be arbitrary (but $\geq 1$ ) although for spin motion in storage rings, the case $d=$ 3 is the most important. To aid the reader we mark the key equations with $\mathrm{a} \star \star \star$ on the left.

As a by-product of the quasiperiodic structure of the solutions we suggest using spectral analysis as a way of "measuring" the spin tune during spin-orbit tracking simulations and thereby complementing other methods already in use $[8,9,12,13,18,23,33]$. Spectral analysis may also lead to a practical method for deciding whether an invariant spin field exists. These ideas are presented in Sec. IX and formalized in Theorems 9.1 and 9.2.

The paper is summarized in Sec. X where our concepts are also related to simulations and used to discuss some popular notions.

For the rest of the paper, apart from Sec. X, we will now adopt a more efficient notation whereby we use the symbols $J=\left(J_{1}, \ldots, J_{d}\right), \omega=\left(\omega_{1}, \ldots, \omega_{d}\right)$ and $\phi=$ $\left(\phi_{1}, \ldots, \phi_{d}\right),\left(J, \omega, \phi \in \mathbb{R}^{d}\right)$ to mean, respectively, the list of orbital actions, orbital tunes, and orbital angles. From now on we will also adopt the frame dependent abbreviations $S=\left(S_{1}, S_{2}, S_{3}\right), \quad \Omega=\left(\Omega_{1}, \Omega_{2}, \Omega_{3}\right)$, and $n=\left(n_{1}, n_{2}, n_{3}\right)$. Generally, if $\phi$ appears as an independent variable in a function, the function will be $2 \pi$-periodic in $\phi_{1}, \ldots, \phi_{d}$. In that case we say for brevity that the function is $2 \pi$-periodic in $\phi$.

In terms of the new notation, the T-BMT equation and the equations of orbital motion are

$$
\begin{gathered}
\dot{S}=\mathcal{A}(\theta, \phi, J) S, \\
\dot{J}=0, \quad \dot{\phi}=\omega(J),
\end{gathered}
$$

where $\mathcal{A}$ is a real skew-symmetric $3 \times 3$ matrix with nonzero elements $\mathcal{A}_{12}=-\mathcal{A}_{21}=-\Omega_{3}, \mathcal{A}_{13}=$ $-\mathcal{A}_{31}=\Omega_{2}$, and $\mathcal{A}_{23}=-\mathcal{A}_{32}=-\Omega_{1}$. The dot over a symbol denotes differentiation with respect to (w.r.t.) $\theta$. Because the $J$ dependence is only parametric, we will often suppress the symbol $J$ in $\omega$ and $\mathcal{A}$, e.g., as in $\mathcal{A}(\theta, \phi)$. Clearly $\mathcal{A}$ is $2 \pi$-periodic in $\theta$ and in $\phi$. For brevity we just say that functions with such periodicity are $2 \pi$-periodic. On the closed orbit, i.e., for $J=0, \mathcal{A}$ is independent of $\phi$. Note that on the torus $J=0$ the angular variables $\phi$ play a largely artificial role because here $\mathcal{A}$ is independent of $\phi$. But their inclusion is very convenient as it allows one to treat all tori on the same basis. Then all definitions, e.g., that of the ISF, apply to all tori.

A function is called $C^{r}$ if the function together with all of its partial derivatives up to and including those of order $r$ are continuous. In this paper we will assume that, for fixed $J, \mathcal{A}$ is a $C^{1}$ function of $(\theta, \phi)$. A $C^{1}$ function will be called smooth. The smoothness of $\mathcal{A}$ corresponds to the fact that, in real storage rings, the magnetic and electric fields are smooth functions of space and time. The labels for the definitions, propositions, theorems, and lemmas are chosen in a way which indicates their relative positions in the text. The ends of the proofs of lemmas, theorems, and propositions, and the ends of definitions and lists of remarks are marked with the symbol $\square$.

\section{GENERAL PROPERTIES OF THE SPIN MOTION}

We begin by establishing some basic components of our formalism.

Clearly (1.5) gives $J(\theta)=J_{0}$ and $\phi(\theta)=\omega\left(J_{0}\right) \theta+\phi_{0}$ where $J_{0}$ and $\phi_{0}$ are the actions and phases at $\theta=0$. Thus an orbit is labeled by $\left(\phi_{0}, J_{0}\right)$. But if we consider a fixed torus $J_{0}$ we often suppress the symbol $J_{0}$. By "a fixed torus $J_{0}$ " we mean that the orbital tune has the value $\omega\left(J_{0}\right)$ and that the spin motion is characterized by the function $\mathcal{A}\left(\theta, \phi, J_{0}\right)$ of $\theta$ and $\phi$. Equation (1.4) thus becomes

$\star \star \star \quad \dot{S}=A\left(\theta ; \phi_{0}, \omega\right) S, \quad S\left(0 ; \phi_{0}, \omega\right)=S_{0}$,

where the real skew-symmetric $A$ is defined by

$$
A\left(\theta ; \phi_{0}, \omega\right):=\mathcal{A}\left(\theta, \omega \theta+\phi_{0}\right) .
$$

As will become clear from Definition 4.1 in Sec. IV, $A\left(\theta ; \phi_{0}, \omega\right)$ is a quasiperiodic function of $\theta$ with the tunes (frequencies) $1, \omega_{1}, \ldots, \omega_{d}$. The solutions of (2.1) can be written as $S\left(\theta ; \phi_{0}, \omega\right)=\Phi\left(\theta ; \phi_{0}, \omega\right) S\left(0 ; \phi_{0}, \omega\right)$ in terms of the principal solution matrix at $\phi_{0}$ which is the $3 \times 3$ matrix $\Phi\left(\theta ; \phi_{0}, \omega\right)$, defined uniquely by the initial value problem

$$
\frac{\partial \Phi\left(\theta ; \phi_{0}, \omega\right)}{\partial \theta}=A\left(\theta ; \phi_{0}, \omega\right) \Phi
$$




$$
\Phi\left(0 ; \phi_{0}, \omega\right)=I
$$

Thus the principal solution matrix at $\phi_{0}$ is the spin transport matrix from the azimuth 0 to the azimuth $\theta$. Occasionally we call a solution $S\left(\theta ; \phi_{0}\right)$ of $(2.1)$ a "spin trajectory" at $\phi_{0}$. The choice $\theta=0$ for the starting azimuth does not imply a loss of generality as can be seen by considering the general initial value problem $S\left(\theta_{0}\right)=S_{0}, \phi\left(\theta_{0}\right)=\phi_{0}$ for (1.4) and (1.5).

Note that $A\left(\theta ; \phi_{0}\right)$ and $\Phi\left(\theta ; \phi_{0}\right)$ are $2 \pi$-periodic in $\phi_{0}$ and that by the smoothness of $A$ the principal solution matrix is a smooth function of $\left(\theta, \phi_{0}\right)[34,35]$. We will sometimes suppress the symbols $\phi_{0}$ and $\omega$ in $S, A$, and $\Phi$. The key property of $\Phi(\theta)$ is that it belongs to $\operatorname{SO}(3)$, i.e.,

$$
\Phi^{T}(\theta) \Phi(\theta)=I=\Phi(\theta) \Phi^{T}(\theta) \quad \text { and } \quad \operatorname{det}[\Phi(\theta)]=1,
$$

as is easily proved using (2.3) and (2.4).

Let $S_{0}^{1}$ and $S_{0}^{2}$ be two initial conditions for (2.1) and let $a \cdot b=a_{1} b_{1}+a_{2} b_{2}+a_{3} b_{3}$ be the real inner product. Then $S^{1}(\theta) \cdot S^{2}(\theta)=\left(\Phi(\theta) S_{0}^{1}\right) \cdot\left(\Phi(\theta) S_{0}^{2}\right)=$ $\left(\Phi^{T}(\theta) \Phi(\theta) S_{0}^{1}\right) \cdot S_{0}^{2}=S_{0}^{1} \cdot S_{0}^{2}$ so that the inner product of any two solutions of (2.1) is conserved. In particular, the length of a spin vector and the angle between any two spin trajectories at the same $\phi_{0}$ is conserved. In addition, it is easy to show that the cross product of two solutions is a solution. In the remainder of this paper we will, for convenience, allow spins $S$ to have arbitrary length.

An interesting property of (2.1) is that knowledge of one solution completely determines $\Phi$ by a simple integration. It is a standard result for linear systems that knowledge of one solution can be used to reduce the dimension by 1 . Here it reduces the dimension by 2 because of the special structure of $\Phi$ in (2.5), as we will now demonstrate.

Let $v^{3}(\theta)$ be a solution of (2.1), i.e., a spin trajectory at $\phi_{0}$, and let $v^{3}$ be of norm 1 . Choose $v^{1}(\theta)$ and $v^{2}(\theta)$ so that

$$
V(\theta):=\left[v^{1}(\theta), v^{2}(\theta), v^{3}(\theta)\right]
$$

is a $\mathrm{SO}(3)$ matrix. One can, for example, require $v^{1}$ and $v^{2}$ to be solutions of $\dot{v}^{k}=\left(v^{3} \times \dot{v}^{3}\right) \times v^{k}(k=1,2)$, whence we can assume that $V(\theta)$ is smooth, i.e., a $C^{1}$ function.

Next we make a transformation $\Phi \rightarrow \Psi$ on (2.3) and (2.4) defined by

$$
\Phi=V(\theta) \Psi
$$

This gives

$$
\dot{\Psi}=C(\theta) \Psi, \quad \Psi(0)=V^{T}(0),
$$

where

$$
C(\theta)=V^{T}(\theta)(A(\theta) V(\theta)-\dot{V}(\theta))
$$

Since $V(\theta) \in \operatorname{SO}(3), \dot{V}^{T}(\theta) V(\theta)=-V^{T}(\theta) \dot{V}(\theta)$ and thus
$C^{T}=V^{T}\left(A^{T} V+\dot{V}\right)=-C$ by the skew symmetry of $A$. Therefore $C$ is skew-symmetric as expected for rotations. The third column of $C$ is

$$
C\left(\begin{array}{l}
0 \\
0 \\
1
\end{array}\right)=V^{T}\left(A v^{3}-\dot{v}^{3}\right)=0
$$

since $v^{3}$ is a solution of (2.1), and the skew symmetry of $C$ yields

$$
C(\theta)=c_{V}(\theta)\left(\begin{array}{ccc}
0 & -1 & 0 \\
1 & 0 & 0 \\
0 & 0 & 0
\end{array}\right)=: c_{V}(\theta) J,
$$

where (2.10) also serves to define $J$. Therefore

$$
\Psi(\theta)=\exp \left(J \int_{0}^{\theta} c_{V}\left(\theta^{\prime}\right) d \theta^{\prime}\right) V^{T}(0),
$$

as is easily checked by differentiation. Finally,

$$
\begin{aligned}
\star \star \star \quad \Phi\left(\theta ; \phi_{0}\right)= & V(\theta) \\
& \times \exp \left(J \int_{0}^{\theta} c_{V}\left(\theta^{\prime}\right) d \theta^{\prime}\right) V^{T}(0) .
\end{aligned}
$$

The exponentials in these equations can be evaluated by noting that

$$
\exp (J \tau)=\left(\begin{array}{ccc}
\cos \tau & -\sin \tau & 0 \\
\sin \tau & \cos \tau & 0 \\
0 & 0 & 1
\end{array}\right)
$$

So we have constructed the complete principal solution matrix by starting from just one solution of (2.1) and assuming the existence of a smooth $V$. This is the result we were aiming for.

Note that by (2.9) and (2.10) we have

$$
\dot{V}(\theta)=A\left(\theta ; \phi_{0}\right) V(\theta)-c_{V}(\theta) V(\theta) J .
$$

It follows that $\dot{v}^{1}=A v^{1}-c_{V} v^{2}$ from which we deduce $c_{V}=v^{2} \cdot\left(A v^{1}-\dot{v}^{1}\right)=v^{2} \cdot\left(\Omega \times v^{1}\right)-v^{2} \cdot \dot{v}^{1}=$ $\Omega \cdot v^{3}+v^{1} \cdot \dot{v}^{2}$. Thus we obtain the useful formula

$$
c_{V}(\theta)=\Omega\left(\theta, \omega \theta+\phi_{0}\right) \cdot v^{3}(\theta)+v^{1}(\theta) \cdot \dot{v}^{2}(\theta) .
$$

In addition, since $\operatorname{Tr}\left[J^{2}\right]=-2$, it follows from (2.13) that $c_{V}=-(1 / 2) \operatorname{Tr}\left[J V^{T}(A V-\dot{V})\right]$.

\section{Remarks}

(1) Equation (2.7) is equivalent to a change of basis for spin whereby $S$ is expressed as $S=V(\theta) \hat{S}=\hat{S}_{1} v^{1}+$ $\hat{S}_{2} v^{2}+\hat{S}_{3} v^{3}$ so that $\hat{S}$ is the spin in the rotating frame represented by the matrix $V$. Moreover, $\dot{\hat{S}}=c_{V}(\theta) J \hat{S}$ so that $\hat{S}(\theta)=\exp \left(J \int_{0}^{\theta} c_{V}\left(\theta^{\prime}\right) d \theta^{\prime}\right) \hat{S}(0)$. Thus $\hat{S}_{3}$ is constant and $\hat{S}$ precesses around $(0,0,1)$ at a nonconstant rate $c_{V}(\theta)$. From (2.14) the rate $c_{V}$ is, as one would expect, just a combination of the projection of $\Omega$ onto $v^{3}$ and the 
rate, $v^{1} \cdot \dot{v}^{2}$, of rotation of $v^{1}$ and $v^{2}$ around $v^{3}$. In our discussion of spin tune in later sections it will be useful to define a frame in which the spin precesses uniformly. From (2.11) we have

$$
\begin{aligned}
S(\theta)= & V(\theta) \exp \left\{J \int_{0}^{\theta}\left(c_{V}\left(\theta^{\prime}\right)-\nu\right) d \theta^{\prime}\right\} \\
& \times \exp (J \nu \theta) V^{T}(0) S(0),
\end{aligned}
$$

where $\nu$ is an arbitrary constant. Thus with the change of basis

$$
\begin{aligned}
S & =U(\theta) \hat{S}, \\
U(\theta) & =V(\theta) \exp \left\{J \int_{0}^{\theta}\left(c_{V}\left(\theta^{\prime}\right)-\nu\right) d \theta^{\prime}\right\},
\end{aligned}
$$

we obtain $\dot{\hat{S}}=\nu J \hat{S}$ and we have defined a frame $U$ whose third column is a spin trajectory and in which spin has a constant precession rate $\nu$. In the following we will be interested in the case where the mean $\bar{c}_{V}=$ $\lim _{T \rightarrow \infty}(1 / T) \int_{0}^{T} c_{V}(\theta) d \theta$ of $c_{V}$ exists and we will choose $\nu=\nu_{V}$ where $\nu_{V}=\bar{c}_{V} \bmod 1$ and is in $[0,1)$. Thus we can write $c_{V}(\theta)=\tilde{c}_{V}(\theta)+\nu_{V}+k_{V}$ where $\tilde{c}_{V}$ represents the fluctuating part of $c_{V}$ with zero mean and where the integer $k_{V}$ is chosen such that $\nu_{V} \in[0,1)$.

(2) The ideas in Remark 1 lead to some precise definitions. On a given torus, let $U(\theta) \in \mathrm{SO}(3)$ be such that the principal solution matrix at $\phi_{0}$ can be written as

$$
\Phi\left(\theta ; \phi_{0}\right)=U(\theta) \exp (J \nu \theta) U^{T}(0),
$$

where $\nu$ is constant and in $[0,1)$. Then $U$ is called a uniform precession frame at $\phi_{0}$ and $\nu$, which is uniquely determined by $U$, is called the uniform precession rate for $U$ and is denoted by $\nu_{s}(U)$. We then call (2.15) a standard form of the principal solution matrix.

Under certain conditions, which will be described in Definition 5.5, $\nu_{s}(U)$ will be called a spin tune. Note that, due to (2.15), $U(\theta)$ is smooth in $\theta$ and satisfies the ordinary differential equation:

$$
\dot{U}(\theta)=A\left(\theta ; \phi_{0}\right) U(\theta)-\nu U(\theta) J,
$$

where $\nu=\nu_{s}(U)$. In particular, by (2.16) the vector described by the third column of $U$ obeys (2.1) so that it is a spin trajectory with unit length. Moreover, for every constant $\nu$ the initial value problem defined by (2.16) and the arbitrary initial matrix $U(0) \in \mathrm{SO}(3)$ has the unique solution $U(\theta)=\Phi\left(\theta ; \phi_{0}\right) U(0) \exp (-J \nu \theta)$. Thus every solution $U(\theta)$ of (2.16) with $U(0) \in \mathrm{SO}(3)$ and $\nu \in$ $[0,1)$ is a UPF at $\phi_{0}$ and its UPR $\nu_{s}(U)$ equals $\nu$, i.e.,

$$
\dot{U}(\theta)=A\left(\theta ; \phi_{0}\right) U(\theta)-\nu_{s}(U) U(\theta) J .
$$

Because $\operatorname{Tr}\left[J^{2}\right]=-2$, for every UPF $U$ one has the useful formula

$$
\nu_{s}(U)=-(1 / 2) \operatorname{Tr}\left[J\left(U^{T} A U-U^{T} \dot{U}\right)\right],
$$

which follows from $(2.17)$. Note that the interval $[0,1)$ is just a matter of choice - any convenient half open interval of length 1 could be chosen, e.g., $(0,1]$.

(3) In this section and in the rest of this paper, the concepts of orthonormal reference frame and $\mathrm{SO}(3)$ matrix are interchangeable. Moreover, the elements of the columns of such a matrix are just the components of the unit coordinate vectors of the corresponding frame as, for example, in (2.6). Thus we will often identify the columns with such vectors.

(4) It can be shown that if $V: \mathbb{R} \rightarrow \mathrm{SO}(3)$ with $V=$ : $\left[v^{1}, v^{2}, v^{3}\right]$ is a smooth and $2 \pi$-periodic function (e.g., a $2 \pi$-periodic UPF), then the fractional part of $\chi:=$ $(1 / 2 \pi) \int_{0}^{2 \pi} d \theta v^{1}(\theta) \cdot \dot{v}^{2}(\theta)$ is independent of $v^{1}$ and $v^{2}$, i.e., the fractional part of $\chi$ only depends on $v^{3}$. If $v^{3}$ were represented in the "spinor formalism" [13] then it would be found that the fractional part of $\chi$ is the geometrical phase of $v^{3}$ in the sense of [36].

Finally, we summarize the basic eigenstructure of an $\mathrm{SO}(3)$ matrix $R$ and its exponential representation in terms of a skew-symmetric matrix $B$ in the following lemma.

Lemma 2.1 (a) Let $R$ be a $3 \times 3$ matrix in $\mathrm{SO}(3)$. Then a real number $\mu \in[0,2 \pi)$ and a $\mathrm{SO}(3)$ matrix $W$ exist such that the spectrum of $R, \lambda(R)$, is the set $\left\{e^{i \mu}, e^{-i \mu}, 1\right\}$ (whence the eigenvalues are on the unit circle) and such that

$$
R W=W\left(\begin{array}{ccc}
\cos \mu & -\sin \mu & 0 \\
\sin \mu & \cos \mu & 0 \\
0 & 0 & 1
\end{array}\right)=W \exp (J \mu)
$$

where in the second equality we used (2.12). Furthermore, any such $W=\left[w^{1}, w^{2}, w^{3}\right]$ satisfies the relations $\quad R\left(w^{1} \pm i w^{2}\right)=\exp (\mp i \mu)\left(w^{1} \pm i w^{2}\right)$ and $R w^{3}=w^{3}$. Also, by (2.19), $R=e^{B \mu}$ where the matrix $B=W J W^{T}$ is skew-symmetric.

(b) Conversely, if $B$ is a real skew-symmetric matrix and if $\lambda(B)=\{i,-i, 0\}$, then a $\mathrm{SO}(3)$ matrix $W$ exists such that $B=W J W^{T}$.

The proof is elementary. See, for example, [37,38]. Note that the relation $R w^{3}=w^{3}$ simply means that $w^{3}$ lies along the "axis of rotation" for $R$.

\section{SPIN MOTION ON THE CLOSED ORBIT}

In this section we consider the case $J=0$, so that $A$ of (2.2) has no $\phi_{0}$ or $\omega$ dependence and is $2 \pi$-periodic and smooth in $\theta$. This case corresponds to the $2 \pi$-periodic motion of the particle on the closed orbit and the following theorem applies.

Floquet theorem: For $J=0$, there exists $a \hat{\nu} \in[0,1)$ and $\hat{W} \in \mathrm{SO}(3)$ such that the principal solution matrix defined by (2.3) and (2.4) is independent of $\phi_{0}$ and can be decomposed as

$\star \star \star \quad \Phi(\theta)=\hat{p}(\theta) \hat{W} \exp (J \hat{\nu} \theta) \hat{W}^{T}$, 
where the matrix $\hat{p}(\theta) \in \mathrm{SO}(3)$ is $2 \pi$-periodic, smooth and where $\hat{p}(0)=I$. Moreover, $\lambda(\Phi(2 \pi))=\left\{e^{i \hat{\nu} 2 \pi}, e^{-i \hat{\nu} 2 \pi}, 1\right\}$.

Proof: Since, at $J=0, \mathcal{A}(\theta, \phi)$ is independent of $\phi$, $\Phi\left(\theta ; \phi_{0}\right)$ is independent of $\phi_{0}$. From Lemma 2.1(a) we know that there exist $\mu \in[0,2 \pi)$ and $\hat{W} \in \mathrm{SO}(3)$ such that $\lambda(\Phi(2 \pi))=\left\{e^{i \mu}, e^{-i \mu}, 1\right\}$ and $\Phi(2 \pi)=e^{\hat{B} \mu}$ where $\hat{B}=\hat{W} J \hat{W}^{T}$. Then with $\mu=2 \pi \hat{\nu}, \quad \hat{\nu} \in[0,1)$, $\lambda(\Phi(2 \pi))=\left\{e^{i \hat{\nu} 2 \pi}, e^{-i \hat{\nu} 2 \pi}, 1\right\}$ and $\Phi(2 \pi)=e^{\hat{B} \hat{\nu} 2 \pi}$. A key property of the principal solution matrix is that

$$
\Phi(\theta+2 \pi)=\Phi(\theta) \Phi(2 \pi)
$$

which we can see by noting that the left-hand side (lhs) of (3.2) is a solution matrix of (2.3) by the $2 \pi$-periodicity of $A$ and the right-hand side (rhs) is a solution matrix of (2.3) since $\Phi$ is. They are equal, by the uniqueness of solutions to the initial value problem for (2.3) and (2.4), since they are equal at $\theta=0$.

Define $\hat{p}$ by $\hat{p}(\theta):=\Phi(\theta) e^{-\hat{B} \hat{v} \theta}$. Clearly $\hat{p}(0)=I$ and $\hat{p}(\theta) \in \mathrm{SO}(3)$ since $\Phi \in \mathrm{SO}(3)$ and $\hat{B}$ is skew-symmetric. The periodicity of $\hat{p}$ is clear because

$$
\begin{aligned}
\hat{p}(\theta+2 \pi) & =\Phi(\theta+2 \pi) \exp (-\hat{B} \hat{\nu}(\theta+2 \pi)) \\
& =\Phi(\theta) \Phi(2 \pi) \exp (-\hat{B} \hat{\nu} 2 \pi) \exp (-\hat{B} \hat{\nu} \theta) \\
& =\hat{p}(\theta),
\end{aligned}
$$

where (3.2) is used at the second equality.

Using the Floquet theorem we now make several remarks concerning the principal solution matrix defined by (2.3) and (2.4) when $J=0$, i.e., when $A$ is independent of $\phi_{0}$ and $2 \pi$-periodic in $\theta$. The " "^" symbol which was specific to the theorem is not needed in the following remarks.

\section{Remarks}

(1) If $\Phi(\theta)=p(\theta) \exp (B \nu \theta)$, where $\nu \in[0,1)$, where the real skew-symmetric matrix $B$ has the spectrum $\lambda(B)=\{i,-i, 0\}$ and where the matrix $p(\theta)$ is $2 \pi$-periodic, then $p, B, \nu$ will be called Floquet parameters. In particular $\nu$ is called a Floquet frequency. Thus the Floquet theorem states that Floquet parameters exist and it implies that the principal solution matrix depends on two frequencies where the Floquet frequency emerges in addition to the circulation tune $\omega_{c}=1$. Note that the Floquet parameter $p(\theta)$ is a smooth element of $\mathrm{SO}(3)$ with $p(0)=I$.

(2) If $p, B, \nu$ are Floquet parameters as defined in Remark 1, then from Lemma 2.1(b) a $W \in \mathrm{SO}(3)$ exists such that $W J W^{T}=B$, whence $\Phi(\theta)=$ $p(\theta) W e^{J \nu \theta}(p(0) W)^{T}$. Thus at every $\phi_{0}, p(\theta) W$ is a $2 \pi$-periodic UPF with UPR $\nu$, as defined in Sec. II. We conclude that every Floquet frequency is a UPR of a $2 \pi$-periodic UPF and that (recall Remark 2 of Sec. II) a $2 \pi$-periodic unit-length function of $\theta$ exists, which is a spin trajectory at every $\phi_{0}$. This is the $n_{0}$ mentioned in the Introduction.
Conversely, if $U$ is a $2 \pi$-periodic UPF, then by (2.15)

$$
\Phi(\theta)=U(\theta) \exp \left(J \nu_{s}(U) \theta\right) U^{T}(0)=: p(\theta) \exp (B \nu \theta),
$$

where $p, B, \nu$, defined by

$$
p(\theta):=U(\theta) U^{T}(0), \quad B:=U(0) J U^{T}(0), \quad \nu=\nu_{s}(U),
$$

fulfill all conditions of Floquet parameters. Thus the UPR of every $2 \pi$-periodic UPF is a Floquet frequency, i.e., for $2 \pi$-periodic UPFs the UPR emerges as a Floquet frequency and thus as an extra frequency of the system. We conclude that the set of Floquet frequencies is identical with the set of UPRs which correspond to $2 \pi$-periodic UPFs.

(3) To study the set of Floquet frequencies in more detail, we first consider two sets, $p, B, \nu$ and $\tilde{p}, \tilde{B}, \tilde{\nu}$, of Floquet parameters, i.e.,

$$
\Phi(\theta)=p(\theta) \exp (B \nu \theta)=\tilde{p}(\theta) \exp (\tilde{B} \tilde{\nu} \theta) .
$$

From (3.4) at $\theta=2 \pi$, we obtain $\Phi(2 \pi)=\exp (2 \pi B \nu)=$ $\exp (2 \pi \tilde{B} \tilde{\nu}), \quad$ so that $\lambda(\Phi(2 \pi))=\left\{e^{2 \pi i \nu}, e^{-2 \pi i \nu}, 1\right\}=$ $\left\{e^{2 \pi i \tilde{\nu}}, e^{-2 \pi i \tilde{\nu}}, 1\right\}$. Thus the set of Floquet frequencies has at most two elements and (due to the Floquet theorem) at least one element. In particular, the set of Floquet frequencies has either one element (which then is equal to 0 ) or it has two elements $\nu, 1-\nu$, both of them positive. Note that a Floquet frequency which is in $[0,1 / 2]$ always exists. Moreover, as we will see when we introduce the general concept of spin tune with Definition 5.5, the set of spin tunes contains this set of Floquet frequencies (see Remark 4 of Sec. VI). This in turn allows us to select one of the Floquet frequencies as a "preferred" spin tune which we denote by $\nu_{0}$. In fact this corresponds to the customary choice in which $e^{i \nu_{0} 2 \pi}$ is an eigenvalue of $\Phi(2 \pi)[14,15]$.

(4) By the definition of Floquet parameters, $\nu=0$ is a Floquet frequency if and only if $\Phi(2 \pi)=I$. From Remark 3 it follows that if 0 is a Floquet frequency, then it is the only Floquet frequency. Furthermore all solutions of (2.1) are $2 \pi$-periodic if and only if $\nu=0$ is a Floquet frequency. It follows by Remark 2 that for the case $\nu=0$ every $2 \pi$-periodic UPF has a zero UPR; see also Remark 4 in Sec. V and Theorem 6.4.

The Floquet theorem does not give a method for constructing the principal solution matrix at $J=0$ since $\hat{p}$ is defined by $\Phi, W$, and $\hat{\nu}$-it is a theorem about its properties. We now construct, by the method pointed out in Remark 1 of Sec. II, a standard form for the principal solution matrix in order to compute Floquet parameters used in, or implied by, other treatments of spin motion on the closed orbit $[15,24,39]$. We first show that the matrix (2.6) exists and can be chosen to be $2 \pi$-periodic in $\theta$.

Let $\xi^{3}$ with length 1 denote the eigenvector for eigenvalue 1 of the $\operatorname{SO}(3)$ matrix $\Phi(2 \pi)$. Then the solution of 
(2.1) with $S_{0}=\xi^{3}$ is $2 \pi$-periodic since $S(\theta)=\Phi(\theta) \xi^{3}=$ $\Phi(\theta) \Phi(2 \pi) \xi^{3}=\Phi(\theta+2 \pi) \xi^{3}=S(\theta+2 \pi)$. We now define $v^{3}(\theta):=\Phi(\theta) \xi^{3}$. To complete the construction of the matrix $V$ we must now construct the $2 \pi$-periodic vectors $v^{1}$ and $v^{2}$. To do this we can, for example, assume that a constant unit vector $e$ exists such that $v^{3}(\theta) \times e$ has no zeros. Then we can define $v^{1}(\theta):=v^{3}(\theta) \times e /\left|v^{3}(\theta) \times e\right|$ and $v^{2}(\theta):=v^{3}(\theta) \times v^{1}(\theta)$. In any case the Floquet theorem ensures the existence of a smooth and $2 \pi$-periodic $\mathrm{SO}(3)$ matrix $V(\theta)$, whose third column is $v^{3}(\theta)$ : just choose a $\mathrm{SO}(3)$ matrix $V(0)$ whose third column is $\xi^{3}$ and then choose $V(\theta)=\hat{p}(\theta) V(0)$.

We now consider an arbitrary smooth $2 \pi$-periodic $\mathrm{SO}(3)$ matrix $V$ whose third column is a solution of (2.1) and we construct a $2 \pi$-periodic UPF and the corresponding standard form for the principal solution matrix. From (2.9) and (2.10) $c_{V}(\theta)$ is $2 \pi$-periodic and can be written as $c_{V}=\bar{c}_{V}+\tilde{c}_{V}$ where, as before, $\bar{c}_{V}$ and $\tilde{c}_{V}$ are the mean and zero-mean parts of $c_{V}$. From Remark 1 of Sec. II we can write

$$
U(\theta)=V(\theta) \exp \left\{J\left(\alpha(\theta)+k_{V} \theta\right)\right\}
$$

where $d \alpha / d \theta=\tilde{c}_{V}$ and where $\nu_{V} \in[0,1)$ and the integer $k_{V}$ are chosen such that $\nu_{V}+k_{V}=\bar{c}_{V}$. The principal solution matrix is then given by (3.3) with $\nu_{s}(U)=\nu_{V}$. Because the mean of $\tilde{c}_{V}$ vanishes, the integral $\int_{0}^{\theta} \tilde{c}_{V}\left(\theta^{\prime}\right) d \theta^{\prime}$ is $2 \pi$-periodic in $\theta$ and thus $\exp (J \alpha(\theta))$ and $U(\theta)$ are $2 \pi$-periodic in $\theta$.

The standard form for the principal solution matrix that we promised is given by the first equality in (3.3), where $U$ is defined by (3.5), and the UPR corresponding to $U$ is given by $\nu_{s}(U)=\bar{c}_{V}-k_{V}$. In (3.5) we have a procedure to calculate $U(\theta)$ and $\nu_{s}(U)$ and thus $\Phi(\theta)$, knowing a periodic solution of (2.1). From $U(\theta)$ and $\nu_{s}(U)$ one can construct Floquet parameters as in Remark 2.

Remarks

(5) From Remark 2 it is clear that the unit-length $2 \pi$-periodic spin trajectory $n_{0}$ exists, and from Remark 4 it is clear that the direction of $n_{0}$ is not unique if and only if $\nu_{0}=0$.

(6) The above construction for $v^{3}(\theta)$ is easily done numerically. First integrate (2.3) from $\theta=0$ to $\theta=2 \pi$ numerically to determine $\Phi(2 \pi)$ and then solve the linear system $\Phi(2 \pi) \xi^{3}=\xi^{3}$. Then $v^{3}$ can be found on a grid of points in $[0,2 \pi]$ by numerically integrating (2.1) with $S_{0}=\xi^{3}$. This is the way that $n_{0}(\theta)$ is constructed in SLIM [14] and other related spin codes.

(7) Since $v^{3}$ is a $2 \pi$-periodic solution of (2.1), so is $-v^{3}$. One can therefore replace $V=\left[v^{1}, v^{2}, v^{3}\right]$ by $V=$ $\left[v^{1},-v^{2},-v^{3}\right]$. By (2.14) it follows that with this replacement $\bar{c}_{V}$ becomes $-\bar{c}_{V}$. Thus if $\left[v^{1}, v^{2}, v^{3}\right]$ leads to $\nu_{V} \in$ $\left(\frac{1}{2}, 1\right)$ then $\left[v^{1},-v^{2},-v^{3}\right]$ leads to $\nu_{V} \in\left(0, \frac{1}{2}\right)$, so that one can choose $V$ in (3.5) so as to put the UPR of $U$ in $\left[0, \frac{1}{2}\right]$. $\square$

\section{QUASIPERIODICITY AND A DIOPHANTINE CONDITION}

In the previous section we set up the Floquet form for the principal solution matrix for the case $J=0$ where $A(\theta)$ is $2 \pi$-periodic. However, one of our aims is to obtain an analogous form when $J \neq 0$, i.e., when $A$ contains the frequency vector $\omega$ in addition to the circulation tune 1 . For this and other purposes we need to introduce the concept of quasiperiodicity.

A periodic function has one basic frequency whereas a quasiperiodic function has a finite number of basic frequencies (tunes) denoted by $\nu=\left(\nu_{1}, \nu_{2}, \ldots, \nu_{k}\right)$. We give the following definition.

Definition 4.1: (a) A function $f: \mathbb{R} \rightarrow \mathbb{R}$ is said to be quasiperiodic with tune vector $\nu$ in $\mathbb{R}^{k}$ if a continuous and $2 \pi$-periodic function $F: \mathbb{R}^{k} \rightarrow \mathbb{R}$ exists and

$\star \star \star \quad f(\theta)=F(\nu \theta)$.

A function $f: \mathbb{R} \rightarrow \mathbb{C}$ is said to be quasiperiodic with tune vector $\nu$ in $\mathbb{R}^{k}$ if its real and imaginary parts are quasiperiodic with tune vector $\nu$ in $\mathbb{R}^{k}$. A real or complex matrix valued function is said to be quasiperiodic with tune vector $\nu$ in $\mathbb{R}^{k}$ if its components are.

(b) The spectrum of a quasiperiodic $f$ is defined by $\Lambda(f):=\{\lambda \in \mathbb{R}: a(f, \lambda) \neq 0\}$, where

$$
a(f, \lambda):=\lim _{T \rightarrow \infty} \frac{1}{T} \int_{0}^{T} f(\theta) \exp (-i \lambda \theta) d \theta .
$$

(c) The mean of $f$ is defined by

$$
\bar{f}:=a(f, 0),
$$

and the zero-mean part of $f$ is denoted by $\tilde{f}$ and defined by $f=: \bar{f}+\tilde{f}$.

The class of functions so defined will be denoted by $\mathcal{Q}(\nu ; k)$, where we refer to $\nu$ as the tune vector and $k$ as the order. If either $\nu$ or $k$ are obvious from the context or not relevant we may omit either or both. We prove that $a(f, \lambda)$ exists in Lemma 4.3(d). Note that for $k=1$ all functions in $Q$ are periodic. A tune vector $\nu$ is said to be nonresonant if the equation

$$
m \cdot \nu:=m_{1} \nu_{1}+\cdots+m_{k} \nu_{k}=0,
$$

where $m \in \mathbb{Z}^{k}$ (i.e., $m$ is a $k$ vector of integers), has $m=0$ as the only solution. If there are nontrivial solutions then $\nu$ is said to be resonant. For some applications, e.g., as in Lemma 4.3(d), a tune vector may be assumed to be nonresonant, since otherwise the order can be reduced, as the following lemma shows.

Lemma 4.2 If $f$ is in $\mathcal{Q}(\nu ; k)$, then a nonresonant $\hat{\nu}$ exists such that $f$ is in $\mathcal{Q}(\hat{\nu} ; \hat{k})$, where $\hat{k} \leq k$.

Proof: Let $f \in \mathcal{Q}(\nu ; k)$ and let $f: \mathbb{R} \rightarrow \mathbb{R}$. Then $f(\theta)=F(\nu \theta)$ where $F: \mathbb{R}^{k} \rightarrow \mathbb{R}$ is continuous and $2 \pi$-periodic. If $\nu$ is nonresonant, then nothing has to be proved. If $\nu=0$, then $f$ is constant, so that 
$f \in \mathcal{Q}(1 ; 1)$. If $\nu$ is resonant and nonzero then $k \geq 2$ and there exists nonzero $m \in \mathbb{Z}^{k}$ such that $m \cdot \nu=0$. If $m_{k} \neq$ 0 , then $\hat{F}: \mathbb{R}^{k-1} \rightarrow \mathbb{R}$, defined by $\hat{F}\left(z_{1}, \ldots, z_{k-1}\right):=$ $F\left(-m_{k} z_{1}, \ldots,-m_{k} z_{k-1}, m_{1} z_{1}+\cdots+m_{k-1} z_{k-1}\right)$, is continuous and $2 \pi$-periodic and $f(\theta)=F(\nu \theta)=\hat{F}(\hat{\nu} \theta)$ where $\hat{\nu}:=-\left(\nu_{1}, \ldots, \nu_{k-1}\right) / m_{k}$. The case $m_{k}=0$ can be dealt with analogously because $m$ is nonzero. We have therefore shown that $f \in \mathcal{Q}(\hat{\nu} ; k-1)$, where $\hat{\nu} \neq$ 0 . If $\hat{\nu}$ is resonant, we can repeat the above procedure until a nonresonant tune vector is obtained. For complex valued functions and matrix valued functions the proof proceeds analogously.

A different proof of Lemma 4.2 can be found in [40], Appendix 3.8.

The order in every $\mathcal{Q}$ can also be increased since the $F$ in (4.1) can be viewed as a function with domain $\mathbb{R}^{k+l}$. Thus for an arbitrary real vector $\mu$ in $\mathbb{R}^{l}, \mathcal{Q}(\nu ; k) \subset$ $\mathcal{Q}(\nu, \mu ; k+l)$. For later use we note that if $a$ is an integer, $\exp (J a \theta)$ is $2 \pi$-periodic in $\theta$ and therefore in $Q(1 ; 1)$.

The class of functions $\mathcal{Q}(\nu)$ obviously depends on $\nu$, but two different $\nu$ can generate the same class. For example, $Q$ is unaltered if $\nu$ is replaced by $\hat{\nu}=Q \nu$ where $Q$ is a matrix with integer entries and determinant \pm 1 , i.e., $f \in \mathcal{Q}(\hat{\nu} ; k)$ if and only if $f \in \mathcal{Q}(\nu ; k)$. To see this let $f \in \mathcal{Q}(\nu ; k)$. Then there exists a function $F$ such that (4.1) holds. Let $H(z):=F\left(Q^{-1} z\right)$. Then $H$ is $2 \pi$-periodic since $Q^{-1}$ has integer entries, and $f \in \mathcal{Q}(\hat{\nu} ; k)$ since $f(\theta)=H(\hat{\nu} \theta)$. The converse is proved in the same way.

The periodic function $F$ in Definition 4.1 has the Fourier series

$$
\sum_{m \in \mathbb{Z}^{k}} F_{m} \exp (i m \cdot z)
$$

where the Fourier coefficients of $F$ are defined by

$$
F_{m}:=\frac{1}{(2 \pi)^{k}} \int_{0}^{2 \pi} \cdots \int_{0}^{2 \pi} F(z) \exp (-i m \cdot z) d z_{1} \cdots d z_{k}
$$

and where $m \cdot z:=m_{1} z_{1}+\cdots+m_{k} z_{k}$. If $F$ is of class $C^{k}$ and if we define

$$
S_{N}(z):=\sum_{\substack{m \in \mathbb{Z}^{k} \\\|m\| \leq N}} F_{m} \exp (\mathrm{im} \cdot z),
$$

then the sequence $\left\{S_{N}\right\}$ converges uniformly to $F$ on $\mathbb{R}^{k}$ ([41], p. 411). Furthermore, the sequence $\left\{f_{N}\right\}$, defined by $f_{N}(\theta):=S_{N}(\nu \theta)$, converges uniformly to $f$. Thus we have the representation

$$
f(\theta)=\lim _{N \rightarrow \infty} f_{N}(\theta)=\lim _{N \rightarrow \infty} \sum_{\substack{m \in k^{k} \\\|m\| \leq N}} F_{m} \exp (i(m \cdot \nu) \theta),
$$

for $f$ in terms of the Fourier coefficients of $F$. Here the norm $\|m\|$ of the integer vector $m$ is the max norm, i.e., $\|m\|:=\max \left\{\left|m_{1}\right|, \ldots,\left|m_{k}\right|\right\}$.
For our definition of quasiperiodicity $F$ is only required to be in $C^{0}$, not in $C^{k}$. Thus in general (4.7) does not apply and we need the following lemma.

Lemma 4.3 (a) Let $F$ be a continuous and $2 \pi$-periodic real or complex matrix valued function on $\mathbb{R}^{k}$. Then the sequence $\left\{\Sigma_{N}\right\}$, defined by

$$
\Sigma_{N}(z):=\sum_{\substack{m \in \mathbb{Z}^{k} \\\|m\| \leq N}} A_{N, m} F_{m} \exp (i m \cdot z)
$$

converges uniformly to $F$ on $\mathbb{R}^{k}$, where

$$
A_{N, m}:=\prod_{n=1}^{k} \frac{N+1-\left|m_{n}\right|}{N+1} .
$$

(b) If $f \in \mathcal{Q}(\nu ; k)$ and if $F$ is a continuous and $2 \pi$-periodic function on $\mathbb{R}^{k}$ such that $f(\theta)=F(\nu \theta)$, then

$$
f(\theta)=\lim _{N \rightarrow \infty} \Sigma_{N}(\nu \theta)=\lim _{N \rightarrow \infty} \sum_{\substack{m \in Z^{k} \\\|m\| \leqslant N}} A_{N, m} F_{m} \exp (i(m \cdot \nu) \theta),
$$

where the convergence is uniform. Thus (4.9) generalizes (4.7) to this less smooth case.

(c) Let $\nu$ be nonresonant under the conditions of Lemma 4.3(b). Then the mean of $f$, defined by (4.3), exists and is given by

$$
\bar{f}=F_{0}:=\frac{1}{(2 \pi)^{k}} \int_{0}^{2 \pi} \cdots \int_{0}^{2 \pi} F(z) d z_{1} \cdots d z_{k}
$$

and the Fourier coefficients $F_{m}$ of $F$ in (4.5) satisfy the relation

$$
\begin{aligned}
F_{m} & =\lim _{T \rightarrow \infty} \frac{1}{T} \int_{0}^{T} f(\theta) e^{-i(m \cdot \nu) \theta} d \theta \\
& =\lim _{T \rightarrow \infty} \frac{1}{T} \int_{0}^{T} F(\nu \theta) e^{-i(m \cdot \nu) \theta} d \theta .
\end{aligned}
$$

In particular, $f=0$ implies $F=0$.

(d) Let $f$ be in $\mathcal{Q}(\nu ; k)$. Then a $(f, \lambda)$ exists for all $\lambda$.

(e) Let $f$ be in $\mathcal{Q}(\nu ; k)$. Then $\Lambda(f) \subset\left\{m \cdot \nu: m \in \mathbb{Z}^{k}\right\}$. Proof of Lemma 4.3(a): See, for example, [42].

Proof of Lemma 4.3(b): A simple consequence of Lemma 4.3(a).

Proof of Lemma 4.3(c): That $\bar{f}$ exists and equals $F_{0}$ is proved, for example, in [43], Sec. 10.3. The proof of (4.11) is similar.

Proof of Lemma 4.3(d): Let $f \in \mathcal{Q}(\nu ; k)$. Then by Lemma 4.2, $f \exp (-i \lambda \cdot) \in \mathcal{Q}(\hat{\nu} ; \hat{k})$, where $\hat{\nu}$ is nonresonant and where $f \exp (-i \lambda \cdot)$ denotes the function which maps $\theta$ to $f(\theta) \exp (-i \lambda \theta)$. Applying Lemma 4.3(c) we conclude that $a(f, \lambda)$ exists for all $\lambda$.

Proof of Lemma 4.3(e): Since $a\left(\Sigma_{N}(\nu \cdot), \lambda\right)$ and $a(f, \lambda)$ exist by Lemma 4.3(d), we have 


$$
\begin{aligned}
& \left|a\left(\Sigma_{N}(\nu \cdot), \lambda\right)-a(f, \lambda)\right| \\
& \quad=\left|a\left(\Sigma_{N}(\nu \cdot)-f, \lambda\right)\right| \\
& \quad=\left|\lim _{T \rightarrow \infty} \frac{1}{T} \int_{0}^{T}\left(\Sigma_{N}(\nu \theta)-f(\theta)\right) \exp (-i \lambda \theta) d \theta\right| \\
& \quad \leq \sup _{\theta}\left|\Sigma_{N}(\nu \theta)-f(\theta)\right|,
\end{aligned}
$$

where we note that $\Sigma_{N}(\nu \cdot)$ and $f$ are bounded functions and where $|\cdot|$ denotes the Euclidean norm. It follows that

$$
\lim _{N \rightarrow \infty} a\left(\Sigma_{N}(\nu \cdot), \lambda\right)=a(f, \lambda)
$$

since the convergence in (4.9) is uniform. If $\lambda \notin$ $\left\{m \cdot \nu: m \in \mathbb{Z}^{k}\right\}$, then $a\left(\Sigma_{N}(\nu \cdot), \lambda\right)=0$ and (4.13) gives $a(f, \lambda)=0$. Thus if $\lambda \notin\left\{m \cdot \nu: m \in \mathbb{Z}^{k}\right\}$, then $\lambda \notin$ $\Lambda(f)$, so that $\Lambda(f) \subset\left\{m \cdot \nu: m \in \mathbb{Z}^{k}\right\}$.

\section{Remarks}

(1) Lemma 4.3(a) is a multidimensional generalization of Fejér's theorem and it shows that a continuous function can be recovered from its Fourier coefficients. For $k=1$, $\Sigma_{N}$ in (4.8) is the Cesàro sum of the sequence $\left\{S_{N}\right\}$. For $k>1$ it is a natural generalization of Cesàro summability and is one of many techniques for summation. The claim in Lemma 4.3(c) that $\bar{f}=F_{0}$, is a "flow" version of Weyl's equidistribution theorem which is one of the main theorems of ergodic theory [26], [27], Chap. 3, and [43]. Of course, if $F$ is in $C^{k}$ then $S_{N}$ and $\Sigma_{N}$ converge to the same function $F$.

(2) Definition 4.1 gives $f$ in terms of $F$, and Lemma 4.3(a) and (4.11) show how to recover $F$ given $f$ with nonresonant $\nu$. Furthermore Lemma 4.3(b) gives a representation for $f$ even when (4.7) is not valid. If $\nu$ is resonant then Lemma 4.3 does not allow $F$ to be recovered. However we can find $\hat{F}$ for the nonresonant tune vector $\hat{\nu}$ of Lemma 4.2.

(3) If $f$ is quasiperiodic, then by Theorem 4.3(d) $\bar{f}$ exists, whence $\tilde{f}:=f-\bar{f}$ is quasiperiodic and the mean of $\tilde{f}$ exists. In particular $\overline{\tilde{f}}=\overline{f-\bar{f}}=0$, which justifies calling $\tilde{f}$ the zero-mean part of $f$.

(4) From (4.8) and (4.11) we see that $F$, for nonresonant $\nu$, is determined by its values on the curve $D^{\prime}:=$ $\{\nu \theta: \theta \in \mathbb{R}\}$. That this is to be expected can be shown as follows. Because $F$ is $2 \pi$-periodic, the knowledge of $F$ at the points of $D^{\prime}$ implies that $F$ is even known at the points of the larger set $\tilde{D}:=\{\nu \theta+2 \pi M: \theta \in \mathbb{R}, M \in$ $\mathbb{Z}^{k}$. But $\tilde{D}$ is dense in $\mathbb{R}^{k}$ since $\nu$ is nonresonant and any continuous function is determined by its values on a dense set.

The following definition will be convenient in some of our proofs.

Definition 4.4: A function $f: \mathbb{R} \rightarrow \mathbb{R}$ is said to be almost periodic if a complex sequence $f_{n}$ and a real sequence $\lambda_{n}$ exist such that the sequence of functions $\sum_{n=1}^{N} f_{n} \exp \left(i \lambda_{n} \theta\right)$ converges uniformly to $f$ as $N \rightarrow \infty$. A function $f: \mathbb{R} \rightarrow \mathbb{C}$ is said to be almost periodic if its real and imaginary parts are. A real or complex matrix valued function is said to be almost periodic if it is almost periodic in each component.

\section{Remark}

(5) Due to Lemma 4.3(b), the quasiperiodic functions form a subset of the almost periodic functions. In contrast to the quasiperiodic functions, the almost periodic functions generally have no finite set of basic frequencies. The Fourier representation of such a function is given by $\sum_{n=1}^{\infty} f_{n} \exp \left(i \lambda_{n} \theta\right)$, where $\left\{\lambda_{n}\right\}$ is the set of frequencies. A quasiperiodic function can be written in this way, but (4.7) is often more convenient for analysis. Almost periodic functions were introduced by $\mathrm{H}$. Bohr and a standard modern treatment can be found in [44], and in summarized form in the appendix of [35].

We have now provided the basic machinery needed to deal with the quasiperiodic functions appearing in the following sections. In the remainder of this section we treat a special aspect of quasiperiodicity, namely the so called small divisor problem, and we show how one solves this problem by using sufficiently differentiable functions. This material will be needed only in Theorems 6.5(c) and 6.5(d).

Integration as in the definition of $\alpha(\theta)$ in Sec. III [see Eq. (3.5)] is, of course, a smoothing operation and the integral of a periodic function with zero mean is periodic. It is perhaps surprising then that the integral of a quasiperiodic function with zero mean is not necessarily quasiperiodic. This is an example of the so-called small divisor problem. To see this let $\nu$ be nonresonant and let $f$ be in $\mathcal{Q}(\nu ; k)$ with zero mean with its associated $F$ in $C^{k}$. Define $g(\theta)=\int_{0}^{\theta} f\left(\theta^{\prime}\right) d \theta^{\prime}$ for $f$ given by (4.7). Because of the uniform convergence in (4.7) we can interchange limit and sum giving

$$
g(\theta)=\lim _{N \rightarrow \infty} \sum_{\substack{m \in \in^{k} \\ 0<\|m\| \leq N}}-i(m \cdot \nu)^{-1} F_{m}\{\exp (i(m \cdot \nu) \theta)-1\} .
$$

However, although $g$ is well defined, it may not be quasiperiodic if $k \geq 2$. The source of the problem is that the divisor $m \cdot \nu$, while not zero, can be arbitrarily small and this can lead to an unbounded $g$, thereby contradicting (4.1). We give such an example in Sec. VIII. To find a sufficient condition for quasiperiodicity we define

$$
G_{N}(z):=\sum_{\substack{m \in \mathbb{Z}^{k} \\ 0<\|m\| \leq N}}-i(m \cdot \nu)^{-1} F_{m}(\exp (i m \cdot z)-1),
$$

and note that $g(\theta)=\lim _{N \rightarrow \infty} G_{N}(\nu \theta)$ so that $G_{N}$ converges on the lines $z=\nu \theta+2 \pi l$ in $\mathbb{R}^{k}$ where $l$ is an integer. Now suppose that $G_{N}$ converges pointwise in $\mathbb{R}^{k}$ to a function $G$. Then $G$ is $2 \pi$-periodic and $g(\theta)=G(\nu \theta)$. Since $g$ is continuous, $G$ is continuous along the lines $z=$ $\nu \theta+2 \pi l$, but Definition 4.1 requires that $G$ be continuous 
everywhere on $\mathbb{R}^{k}$. So we need a condition that ensures both pointwise convergence of $G_{N}$ and the continuity of the limit. Uniform convergence guarantees both, and a sufficient condition for this is a so called Diophantine condition which places a condition on how close $m \cdot \nu$ can be to zero.

In the following we will be concerned with the tune vector $\nu=(1, \omega)$ where 1 is the circulation tune and $\omega \in$ $\mathbb{R}^{d}$ is the orbital tune. Thus we formulate the Diophantine condition and the convergence of $G_{N}$ in terms of $\nu=$ $(1, \omega)$ and $k=d+1$. See [40], Appendix 4, for some details on Diophantine conditions and [45] for further discussion and its use in another beam dynamics context. Definition 4.5: The tune vector $\omega \in \mathbb{R}^{d}$ is said to satisfy a Diophantine condition if, for positive $\tau$, $\omega$ is in the Diophantine set $\Omega(\tau):=\bigcup_{\gamma \in(0,1]} \Omega(\tau, \gamma)$ where $\Omega(\tau, \gamma)$ is the set

$$
\left\{\omega \in \mathbb{R}^{d}:|m \cdot(1, \omega)| \geq \gamma\|m\|^{-\tau}, m \in \mathbb{Z}^{d+1}, m \neq 0\right\} .
$$

Note that the symbol $\Omega$ is also used in the Introduction for the precession vector. However its meaning should be clear from the context.

It follows from the definition that $\Omega^{c}(\tau, \gamma)=$ $\bigcup_{m \in \mathbb{Z}^{d+1} \backslash\{0\}} Z(\tau, \gamma, m), \quad$ where $\quad Z(\tau, \gamma, m):=\{\omega \in$ $\left.\mathbb{R}^{d}:|m \cdot(1, \omega)|<\gamma\|m\|^{-\tau}\right\}$. For fixed $(\tau, \gamma)$ and for each $m$ the "resonant zone" $Z(\tau, \gamma, m)$ is either empty or a thickened $(d-1)$ dimensional plane centered on the resonant plane, $m \cdot(1, \omega)=m_{0}+m_{1} \omega_{1}+\cdots+$ $m_{d} \omega_{d}=0$, with thickness proportional to $\gamma$. For example, when $d=1,\|m\|=1$ and $m_{1} \neq 0$ the corresponding zones are intervals centered on the three points in $\mathbb{R}$ : $\omega_{1}=\eta$ where $\eta \in\{-1,0,1\}$, each with thickness $2 \gamma$. When $d=2,\|m\|=1$ and $\left(m_{1}, m_{2}\right) \neq 0$ the corresponding zones are centered on the twelve lines in $\mathbb{R}^{2}: \omega_{1}=$ $\eta, \omega_{2}=\eta, \omega_{2}= \pm \omega_{1}+\eta$ where $\eta \in\{-1,0,1\}$ with thickness either $2 \gamma$ or $\sqrt{2} \gamma$. More generally, if $m=$ $\left(m_{0}, \hat{m}\right)$ with $m_{0} \in \mathbb{Z}$ and $\hat{m} \in\left(\mathbb{Z}^{d} \backslash\{0\}\right)$ then one can show by using rotations in $\mathbb{R}^{d}$ that $Z(\tau, \gamma, m)$ can be rotated into the set $\left\{\omega \in \mathbb{R}^{d}:\left|\frac{m_{0}}{|\hat{m}|}+\omega_{1}\right|<\frac{\gamma\|m\|^{-\tau}}{|\hat{m}|}\right\}$. Thus the thickened $(d-1)$ dimensional plane $Z(\tau, \gamma, m)$ has thickness $2 \gamma\|m\|^{-\tau} /|\hat{m}|$. For $m$ such that $\hat{m}=0$ the resonance condition cannot be satisfied and we have $Z(\tau, \gamma, m)=\emptyset$. Note also that $Z(\tau, \gamma, m)$ is undefined for $m=0$.

Definition 4.6 (orbital resonance): We say that the torus at $J$ is off orbital resonance if $(1, \omega(J))$ is nonresonant (otherwise we say that it is on orbital resonance) and this is certainly the case if $\omega(J) \in \Omega(\tau)$. Usually, (1.5) is said to be resonant if $\omega$ is resonant. Our usage is different because our basic system is (2.1), which includes the circulation tune, 1 .

We can now interpret $\Omega(\tau, \gamma)$ as the closed set in $\mathbb{R}^{d}$ constructed by successively removing the open resonance zones, corresponding to the resonance planes $m \cdot(1, \omega)=$
0 , with increasing $\|m\|$. Thus its construction is similar to the construction of a Cantor set. The resonance planes are dense in $\mathbb{R}^{d}$ and thus $\Omega(\tau, \gamma)$ is small in the sense that it has an empty interior. However, we will show in the proof of Lemma 4.8 that it is large in the sense that for $\tau>d+$ 1 the Lebesgue measure of its complement relative to $\mathbb{R}^{d}$ is proportional to $\gamma$ [in the sense of (4.23)] which can be arbitrarily small. We could take our Diophantine set to be $\Omega(\tau, \gamma)$ for $\gamma$ small as in [45]. Here we take the larger set $\Omega(\tau)$.

Now if $\omega \in \Omega(\tau, \gamma)$ then $\left|(m \cdot(1, \omega))^{-1} F_{m}\right| \leq$ $\gamma^{-1}\|m\| \tau\left|F_{m}\right|$ and thus $G_{N}$ converges uniformly if $\sum\|m\|^{\tau}\left|F_{m}\right|$ converges. Thus the Diophantine condition leads to a simple sufficient condition for the quasiperiodicity of $g$. In this context we now state and prove the following lemma which addresses the differentiability of $F$.

Lemma 4.7 Let $F: \mathbb{R}^{d+1} \rightarrow \mathbb{R}$ be of class $C^{n}$ and $2 \pi$-periodic and let $F_{0}=0$. Let $\omega \in \Omega(\tau)$ where $0<$ $\tau<n-d-2$. Then $G_{N}: \mathbb{R}^{d+1} \rightarrow \mathbb{R}$, given by (4.15) with $\nu=(1, \omega)$, converges uniformly on $\mathbb{R}^{d+1}$ to a smooth function $G$ which is $2 \pi$-periodic. Moreover, $\nabla G(z) \cdot(1, \omega)=F(z)$.

Proof: Because a constant $M \geq 0$ exists such that $\|m\|^{n}\left|F_{m}\right| \leq M$ (see [41], p. 409), we have, for every $\tau>0$,

$$
\sum_{\substack{m \in \mathbb{Z}^{d+1} \\ 0<\|m\| \leq N}}\|m\|^{\tau}\left|F_{m}\right| \leq M \sum_{\substack{m \in \mathbb{Z}^{d+1} \\ 0<\|m\| \leq N}}\|m\|^{\tau-n}=M \sum_{j=1}^{N} j^{\tau-n} \sum_{\substack{m \in \mathbb{Z}^{d+1} \\\|m\|=j}} 1 .
$$

A combinatorial argument gives

$$
\sum_{\substack{m \in \mathbb{Z}^{d+1} \\\|m\|=j}} 1 \leq 3^{d+1}(d+1) j^{d} .
$$

Moreover, if $\omega \in \Omega(\tau, \gamma)$, then $\left|(m \cdot(1, \omega))^{-1}\right| \leq$ $\gamma^{-1}\|m\|^{\tau}$ and (4.16) and (4.17) give

$$
\sum_{\substack{m \in \mathbb{Z}^{d+1} \\ 0<\|m\| \leq N}}\left|(m \cdot(1, \omega))^{-1} F_{m}\right| \leq 3^{d+1}(d+1) M \gamma^{-1} \sum_{j=1}^{N} j^{\tau-n+d} .
$$

Since $\tau<n-d-2$ we conclude that the lhs of (4.18) converges as $N \rightarrow \infty$ for every $\omega \in \Omega(\tau)$. It follows that $G_{N}$ converges uniformly to a continuous function $G$ which is $2 \pi$-periodic.

From (4.15) we have

$$
\nabla G_{N}(z)=\sum_{\substack{m \in \mathbb{Z}^{d+1} \\ 0<\|m\| \leq N}}(m \cdot(1, \omega))^{-1} F_{m} \exp (\mathrm{im} \cdot z) m .
$$

Then, if $\omega \in \Omega(\tau, \gamma)$ and repeating the above argumentation, we find 


$$
\begin{aligned}
\left|\nabla G_{N}(z)\right| & \leq \sum_{\substack{m \in \mathbb{Z}^{d+1} \\
0<\|m\| \leq N}}\left|(m \cdot(1, \omega))^{-1} F_{m}\right||m| \\
& \leq \sqrt{d+1} \sum_{\substack{m \in \mathbb{Z}^{d+1} \\
0<\|m\| \leq N}}\left|(m \cdot(1, \omega))^{-1} F_{m}\right|\|m\| \\
& \leq 3^{d+1}(d+1)^{3 / 2} M \gamma^{-1} \sum_{j=1}^{N} j^{\tau+1-n+d} .
\end{aligned}
$$

Thus, if $\omega \in \Omega(\tau), \nabla G_{N}$ converges uniformly and a standard result (see [46], Sec. 8.6.3, [47], p. 117) means that $G$ is $C^{1}$ and

$$
\nabla G(z)=\lim _{N \rightarrow \infty} \sum_{\substack{m \in \mathbb{Z}^{d+1} \\ 0<\|m\| \leq N}}(m \cdot(1, \omega))^{-1} F_{m} \exp (i m \cdot z) m .
$$

It follows that $\nabla G(z) \cdot(1, \omega)=\lim _{N \rightarrow \infty} \nabla G_{N}(z)$. $(1, \omega)=F(z)$ since, with $n \geq d+1$, the $S_{N}$ in (4.6) converge.

\section{Remark}

(6) To prove (4.17) we define the sets $s:=\{m \in$ $\left.\mathbb{Z}^{d+1}:\|m\|=j\right\}$ and $s_{i}:=\left\{m \in s:\left|m_{i}\right|=j\right\}$. It follows that $s=\bigcup_{i=1}^{d+1} s_{i}$ and that $s_{i}$ contains $2(2 j+1)^{d}$ elements. Then $s$ contains no more than $2(d+1)(2 j+1)^{d}$ elements and, because

$$
2(2 j+1)^{d} \leq 3(2 j+1)^{d} \leq 3(3 j)^{d}=3^{d+1} j^{d},
$$

we conclude that $s$ contains no more than $3^{d+1}(d+1) j^{d}$ elements, thus proving (4.17).

Lemma 4.7 provides the basic framework that we need for discussing the uniform convergence of the sequence $G_{N}$. In particular it shows that as $n$ increases beyond $\tau+$ $d+2$ the small divisor problem loses much of its potency. This comes as no surprise because the inequality $\|m\|^{n}\left|F_{m}\right| \leq M$ implies that the Fourier coefficients decrease with increasing $\|m\|$ more rapidly as $n$ increases. Then with growing $n$ the small divisor in (4.15) can come closer to zero without destroying the convergence.

However, although Lemma 4.7 takes the mystery out of the working of the Diophantine condition, it puts the burden on determining which $\omega$ are in the set $\Omega(\tau)$ and it is not so easy to decide, off orbital resonance, if $\omega$ is in $\Omega(\tau)$. But some relief comes from the following lemma, which shows that if $\tau>d+1$ then the complement, $\Omega^{c}(\tau)$, of $\Omega(\tau)$ is a small set in terms of Lebesgue measure. If in addition $\tau<n-d-2$, the sequence $G_{N}$ converges uniformly for almost every $\omega$. For these two conditions to be consistent we thus need $n>2 d+3$. These results will be central to the statement and proof of Theorems 6.5(c) and 6.5(d).
Lemma 4.8 If $\tau>d+1$, then $\mu\left(\Omega^{c}(\tau)\right)=0$, where $\mu$ denotes the Lebesgue measure.

Proof: Let $B(R):=\left\{\omega \in \mathbb{R}^{d}:|\omega| \leq R\right\}$ and define $\widetilde{S}(R, m):=\mathcal{Z}(\tau, \gamma, m) \cap B(R)$. Then from Definition 4.5, $\Omega^{c}(\tau, \gamma) \cap B(R)=\bigcup_{m \in \mathbb{Z}^{d+1} \backslash\{0\}} \Im(R, m)$. Note that $Z(\tau, \gamma, m)$ [hence $\subseteq(R, m)$ ] is undefined for $m=0$.

We will show below that

$$
\mu(\widetilde{S}(R, m)) \leq 2 \gamma R^{d-1} \alpha(d-1)\|m\|^{-\tau},
$$

where $\alpha(n):=\pi^{n / 2} / \Gamma(n / 2+1)$ [note that $\alpha(d)$ is the volume of $B(1)]$.

Assuming the validity of (4.22),

$$
\mu\left(\Omega^{c}(\tau, \gamma) \cap B(R)\right) \leq 2 \gamma R^{d-1} \alpha(d-1) \sum_{m \in \mathbb{Z}^{d+1} \backslash\{0\}}\|m\|^{-\tau} .
$$

As before [see (4.17)]

$\sum_{m \in \mathbb{Z}^{d+1} \backslash\{0\}}\|m\|^{-\tau}=\sum_{j=1}^{\infty} j^{-\tau} \sum_{\substack{m \in \mathbb{Z}^{d+1} \\\|m\|=j}} 1 \leq 3^{d+1}(d+1) \sum_{j=1}^{\infty} j^{d-\tau}$.

Therefore

$$
\begin{aligned}
& \mu\left(\Omega^{c}(\tau, \gamma) \cap B(R)\right) \\
& \quad \leq 2 \gamma R^{d-1} \alpha(d-1) 3^{d+1}(d+1) \sum_{j=1}^{\infty} j^{d-\tau},
\end{aligned}
$$

and for $\tau>d+1$ the series converges. Now $\Omega^{c}(\tau, 1 / p)$ decreases monotonically to $\Omega^{c}(\tau)$ as the positive integer $p \rightarrow \infty$. Therefore

$$
\mu\left(\Omega^{c}(\tau) \cap B(R)\right)=\lim _{p \rightarrow \infty} \mu\left(\Omega^{c}(\tau, 1 / p) \cap B(R)\right)=0
$$

by continuity and the finiteness of Lebesgue measure restricted to $B(R)$ where the second equality follows from (4.23). Since this is true for all $R$ we obtain the required result.

To prove (4.22) we first recall that $Z(\tau, \gamma, m)$ is empty if $m=\left(m_{0}, 0, \ldots, 0\right)$. Thus $\widetilde{S}(R, m)$ can only be nonempty if $m=\left(m_{0}, \hat{m}\right)$ with $m_{0} \in \mathbb{Z}$ and $\hat{m} \in\left(\mathbb{Z}^{d} \backslash\{0\}\right)$ so that we only have to consider this case. Using the fact that volumes are invariant under rotations, it follows from the remarks after Definition 4.5 that

$$
\mu(\Im(R, m)) \leq \mu\left(\left\{\omega \in B(R):\left|\frac{m_{0}}{|\hat{m}|}+\omega_{1}\right|<\gamma\|m\|^{-\tau}\right\}\right),
$$

where we also used the fact that $1 /|\hat{m}| \leq 1$. If $d=1$ then (4.24) and the fact that $\alpha(0)=1$ immediately yield (4.22). Furthermore, for $d \geq 2$, we conclude from (4.24) that 


$$
\begin{aligned}
\mu(\widetilde{S}(R, m)) & \leq \mu\left(\left\{\omega \in \mathbb{R}^{d}: \sqrt{\omega_{2}^{2}+\cdots+\omega_{d}^{2}} \leq R,\left|\frac{m_{0}}{|\hat{m}|}+\omega_{1}\right|<\gamma\|m\|^{-\tau}\right\}\right) \\
& =\mu\left(\left\{\omega \in \mathbb{R}^{d}: \sqrt{\omega_{2}^{2}+\cdots+\omega_{d}^{2}} \leq R,\left|\omega_{1}\right|<\gamma\|m\|^{-\tau}\right\}\right)=2 \gamma R^{d-1} \alpha(d-1)\|m\|^{-\tau},
\end{aligned}
$$

where the rhs is just the volume of the cylinder in $\mathbb{R}^{d}$ with height $2 \gamma\|m\|^{-\tau}$ and radius $R$ and where in the first equation we used the fact that volumes are invariant under translations. This completes the proof.

\section{INTRODUCTION TO THE QUASIPERIODICITY OF THE SPIN MOTION: DEFINITION OF THE SPIN TUNE}

We can now continue the study of the principal solution matrix $\Phi\left(\theta ; \phi_{0}, \omega\right)$ for (2.1) which is defined by the initial value problem

$$
\frac{\partial \Phi}{\partial \theta}=A\left(\theta ; \phi_{0}, \omega\right) \Phi, \quad \Phi\left(0 ; \phi_{0}, \omega\right)=I,
$$

where

$$
A\left(\theta ; \phi_{0}, \omega\right):=\mathcal{A}\left(\theta, \omega \theta+\phi_{0}\right) .
$$

In the language of Sec. IV, $A\left(\cdot ; \phi_{0}, \omega\right)$ is quasiperiodic in $\mathcal{Q}(1, \omega ; d+1)$. One of the aims of this paper is to define the spin tune for this system. We are guided by the special case $J=0$ of Sec. III and therefore, and as will become clear below, our emphasis in this section is on principal solution matrices, $\Phi$, where $\Phi\left(\cdot ; \phi_{0}, \omega\right)$ is quasiperiodic and can be written in the standard form,

$$
\star \star \star \quad \Phi\left(\theta ; \phi_{0}, \omega\right)=U(\theta) \exp (J \nu \theta) U^{T}(0),
$$

where $U$ is a quasiperiodic $\mathrm{SO}(3)$ matrix in $\mathcal{Q}(1, \omega ; d+$ $1)$ and $\nu \in[0,1)$ and both may depend on $\left(\phi_{0}, \omega\right)$. In that case all solutions of (2.1) are in $\mathcal{Q}(1, \omega, \nu ; d+2)$ with a very simple frequency structure in the tune $\nu$. Such principal solution matrices fulfill a generalized Floquet theorem.

There has been extensive study of the equation $\partial \Phi / \partial \theta=A(\theta ; y) \Phi$, where the matrix $A(\cdot ; y)$ is almost periodic and $y$ is a vector of parameters, one goal being to find conditions under which almost periodic solutions exist (see, for example, $[44,48]$ ). However, our problem (5.1) is quite special because of the parameter dependence of $A$ induced by $\mathcal{A}(\theta, \phi)$ in (5.2). In fact, for every integer $N$ we have

$$
A\left(\theta+2 \pi N ; \phi_{0}, \omega\right)=A\left(\theta ; 2 \pi N \omega+\phi_{0}, \omega\right),
$$

which follows from (5.2) and the $2 \pi$-periodicity of $\mathcal{A}(\theta, \phi)$ in $\theta$. We will return to this in Sec. IX where we will see that condition (5.4) has useful consequences for the spectrum $\Lambda\left(\Phi\left(\cdot ; \phi_{0}, \omega\right)\right)$ of the principal solution matrix.

The case $J=0$ where $A\left(\cdot ; \phi_{0}, \omega\right)$ is $2 \pi$-periodic, i.e., in $\mathcal{Q}(1 ; 1)$ and independent of $\phi_{0}$ and $\omega$, was discussed in
Sec. III. There we found a solution of $(2.1)$ in $\mathcal{Q}(1 ; 1)$, namely $v^{3}(\theta)=\hat{p}(\theta) \xi^{3}$. All other linearly independent solutions are in $\mathcal{Q}(1, \nu ; 2)$. The latter follows directly from the Floquet theorem, but more importantly also from the construction of the $2 \pi$-periodic UPF using $v^{3}$ which led, with $U$ in $\mathcal{Q}(1 ; 1)$, to the representation (5.3). It is this construction that points the way for a generalization of the Floquet theorem to $A \in \mathcal{Q}(1, \omega ; d+1)$.

We begin with the following proposition.

Proposition 5.1 Consider the initial value problem

$$
\dot{S}=A(\theta) S, \quad S(0)=S_{0},
$$

with a real skew-symmetric $3 \times 3$ matrix $A \in Q(\nu ; k)$ and make the following assumptions.

(a) Equation (5.5) has a nonzero solution $v^{3}$ in $\mathcal{Q}(\nu ; k)$.

(b) There exists a smooth $\mathrm{SO}(3)$ matrix $V=$ $\left[v^{1}, v^{2}, v^{3}\right]$ in $Q(\nu ; k)$.

(c) Let $c:=v^{2} \cdot\left(A v^{1}-\dot{v}^{1}\right)$. Since $c \in \mathcal{Q}(\nu ; k)$ its mean $\bar{c}$ and zero-mean part $\tilde{c}$ exist (recall Remark 3 of Sec. IV). We assume that $\int_{0}^{\theta} \tilde{c}\left(\theta^{\prime}\right) d \theta^{\prime} \in \mathcal{Q}(\nu ; k)$.

Then all solutions of $(5.5)$ are in $\mathcal{Q}\left(\nu, \nu_{k+1} ; k+1\right)$ where $\nu_{k+1}=\bar{c} \bmod 1$ and $\nu_{k+1} \in[0,1)$.

Proof: From (c) there is an integer $l$ such that $c(\theta)=$ $\bar{c}+\tilde{c}(\theta)=\nu_{k+1}+l+\tilde{c}(\theta)$ and by the same calculation that leads to (2.11) the principal solution matrix for (5.5) is

$\Phi(\theta)=V(\theta) \exp \left\{J\left(\int_{0}^{\theta} \tilde{c}\left(\theta^{\prime}\right) d \theta^{\prime}+l \theta\right)\right\} \exp \left(J \nu_{k+1} \theta\right) V^{T}(0)$.

Clearly $\Phi(\theta) \in \mathcal{Q}\left(\nu, \nu_{k+1} ; k+1\right)$ and thus all solutions are quasiperiodic as stated.

However as pointed out in Sec. IV, even if conditions (a) and (b) are fulfilled, condition (c) might still be problematic since the integral of a zero-mean quasiperiodic function may not be quasiperiodic.

To continue our discussion of the quasiperiodic structure of the principal solution matrix defined in (5.1) and its potential representation in (5.3), we elaborate on the definition of UPF and UPR from Remark 2 in Sec. II.

Definition 5.2 (UPF and UPR): Consider (5.1) for a fixed $\phi_{0}$ on a fixed torus $J_{0}$. If $\nu \in[0,1)$ and $U \in \mathrm{SO}(3)$ exist such that the principal solution matrix at $\phi_{0}$ can be written as in (5.3), then the orthonormal coordinate system represented by $U$ is called a UPF (uniform precession frame) at $\phi_{0}$ and $\nu=: \nu_{s}(U)$ is called the UPR (uniform precession rate) for $U$. If $U \in \mathcal{Q}(1, \omega ; d+1)$, then it is called proper. A UPR is called proper if it corresponds to a proper UPF. The set of all proper UPFs at $\phi_{0}$ is denoted 
by $\mathfrak{U}\left(\phi_{0}\right)$ and the set of all proper UPFs on the torus is given by $\mathfrak{U}:=\bigcup_{\phi_{0} \in \mathbb{R}^{d}} \mathfrak{U}\left(\phi_{0}\right)$. In addition we denote the set of all proper UPRs at $\phi_{0}$ by $\Xi\left(\phi_{0}\right)$ and then the set of all proper UPRs on the torus is given by $\Xi:=\bigcup_{\phi_{0} \in \mathbb{R}^{d}} \Xi\left(\phi_{0}\right)$.

For further background material see Remarks 1 and 2 in Sec. II. Recall that in Definition 5.2 "on a fixed torus $J_{0}$ " means that the orbital tune has the value $\omega\left(J_{0}\right)$ and that the spin motion is characterized by the function $\mathcal{A}\left(\cdot, \cdot, J_{0}\right)$. Recall also that we often suppress the symbol $J_{0}$. From the discussion in Sec. II we know that UPFs always exist. However, the existence of a proper UPF imposes additional constraints. For example, Proposition 5.1 gives rise to the existence of a proper UPF for (5.1) (and a proper UPR) but the conditions are rather severe. If a proper UPF $U$ at $\phi_{0}$ exists, then clearly $\Phi\left(\cdot ; \phi_{0}\right) \in \mathcal{Q}\left(1, \omega, \nu_{s}(U) ; d+2\right)$. However, it is unknown if the converse holds although this is a reasonable conjecture. Note that for each proper UPR there is an infinite number of proper UPFs: if $U$ is a proper UPF at $\phi_{0}$, then $U \exp (J y)$ is a proper UPF at $\phi_{0}$ if $y$ is a real constant.

It is easy to see that if a proper UPF $U$ exists at a given $\phi_{0}$, then for all $\nu \in[0,1)$ such that $\nu=$ $\pm \nu_{s}(U)+j_{0}+j \cdot \omega$, where $\left(j_{0}, j\right) \in \mathbb{Z} \times \mathbb{Z}^{d}, \tilde{U}(\theta):=$ $\left[u^{1}(\theta), \pm u^{2}(\theta), \pm u^{3}(\theta)\right] \exp \left(-J\left(j_{0}+j \cdot \omega\right) \theta\right) \quad$ is a proper UPF at that $\phi_{0}$ with $\nu_{s}(\tilde{U})=\nu$. This motivates the definition of an equivalence relation where $\nu_{1}$ and $\nu_{2}$ in $[0,1)$ are said to be equivalent, and we write $\nu_{1} \sim \nu_{2}$, if and only if there exist $\left(\varepsilon, j_{0}, j\right) \in\{-1,1\} \times \mathbb{Z} \times \mathbb{Z}^{d}$ such that $\nu_{2}=\varepsilon \nu_{1}+j_{0}+j \cdot \omega$. The equivalence relation partitions the interval into equivalence classes $[\nu]:=\{\mu \in$ $[0,1): \mu \sim \nu\}$ such that $\left[\nu_{1}\right]=\left[\nu_{2}\right]$ if and only if $\nu_{1} \sim$ $\nu_{2}$ and $\left[\nu_{1}\right] \cap\left[\nu_{2}\right]=\emptyset$ if and only if $\nu_{1} \nsucc \nu_{2}$. We note that if $\omega$ has one irrational component then each $[\nu]$ is a dense subset of $[0,1)$. To see this suppose that $\omega_{1}$ is irrational. Then $\varepsilon \nu+j_{1} \omega_{1} \bmod 1$ is dense as $j_{1}$ varies over $\mathbb{Z}$ so that $\mu=\varepsilon \nu+j_{0}+j \cdot \omega$ is dense in $[0,1)$ as $j_{0}$ and $j$ vary.

From the above motivation for the definition of equivalence it is clear that if $\nu_{1} \in \Xi\left(\phi_{0}\right)$ then the equivalence $\nu_{1} \sim \nu_{2}$ implies $\nu_{2} \in \Xi\left(\phi_{0}\right)$, i.e., $\left[\nu_{1}\right] \subset \Xi\left(\phi_{0}\right)$. Now suppose that $U_{1}$ and $U_{2}$ are in $\mathfrak{H}\left(\phi_{0}\right)$ and that $\nu_{s}\left(U_{1}\right)=$ $\nu_{1}$. Then $\exp \left(J \nu_{s}\left(U_{2}\right) \theta\right)=U_{2}^{T}(\theta) U_{1}(\theta) \exp \left(J \nu_{s}\left(U_{1}\right) \theta\right)$. $U_{1}^{T}(0) U_{2}(0)$ so that the lhs is in $\mathcal{Q}\left(1, \omega, \nu_{s}\left(U_{1}\right) ; d+2\right)$. Thus $\nu_{s}\left(U_{2}\right)=j_{0}+j \cdot \omega+j_{d+2} \nu_{s}\left(U_{1}\right)$ by Lemma 4.3(e). It is plausible that $j_{d+2}= \pm 1$. If that is so, then $\nu_{1}=\nu_{s}\left(U_{1}\right) \sim \nu_{s}\left(U_{2}\right)$. Then since $U_{2}$ is an arbitrary member of $\mathfrak{U}\left(\phi_{0}\right), \Xi\left(\phi_{0}\right) \subset\left[\nu_{1}\right]$. In fact this is the case, and the joint conditions $\left[\nu_{1}\right] \subset \Xi\left(\phi_{0}\right)$ and $\Xi\left(\phi_{0}\right) \subset$ $\left[\nu_{1}\right]$ can be embodied in a theorem.

Theorem 5.3 If $\Xi\left(\phi_{0}\right)$ is nonempty with an element $\nu$, then $[\nu]=\Xi\left(\phi_{0}\right)$.

Although all proper UPRs are equivalent at a given $\phi_{0}$ on the torus $J_{0}$, it is not true in general that all proper UPRs on the torus are equivalent. But if they are, we then say that the torus is well-tuned and we call any $\nu \in \Xi$ a spin tune. Since this situation is central to this paper, we delay the proof of Theorem 5.3 in order to formalize this definition.

Definition 5.4 (well-tuned): A torus is said to be welltuned if and only if the following two conditions hold.

(a) $\Phi\left(\cdot ; \phi_{0}\right)$ has a proper UPF for each $\phi_{0} \in \mathbb{R}^{d}$, i.e., each $\mathfrak{H}\left(\phi_{0}\right)$ is nonempty.

(b) Let $U$ and $\hat{U}$ be in $\mathfrak{U}$, then $\nu_{s}(U) \sim \nu_{s}(\hat{U})$. Thus for every $\nu \in \Xi,[\nu] \supset \Xi$ and, by Theorem 5.3, $[\nu] \subset \Xi$ so that then $[\nu]=\Xi$.

A torus that is not well-tuned is called ill-tuned.

Note that by Theorem 5.3 a torus is well-tuned if and only if the $\Xi\left(\phi_{0}\right)$ have an element in common. This criterion is very convenient and we will use it, for example, in the proof of Theorem 6.3(a). Note also that $\Xi\left(\phi_{0}\right)$ always contains at most countably many elements. In particular, if a torus is well-tuned then $\Xi$ contains at most countably many elements.

Definition 5.5 (spin tune): Let a torus $J_{0}$ be well-tuned, then each element of $\Xi$ is called a spin tune. Thus for each spin tune $\nu,[\nu]=\Xi$.

To prove Theorem 5.3 we need the following lemma.

Lemma 5.6 Let $\exp \left(\right.$ ic $\left._{0} \theta\right)$, where $c_{0}$ is a real constant, be in $Q(\nu ; k)$ with $k \geq 1$. Then there exists $n \in \mathbb{Z}^{k}$ such that $c_{0}=n \cdot \nu$. Moreover $n$ is unique if $\nu$ is nonresonant.

Proof: First of all we observe that

$$
\Lambda\left(\exp \left(i c_{0} \cdot\right)\right)=\left\{c_{0}\right\} .
$$

Also, by Lemma 4.3(e) we see that $\Lambda\left(\exp \left(i c_{0} \cdot\right)\right) \subset$ $\left\{m \cdot \nu: m \in \mathbb{Z}^{k}\right\}$. Hence by (5.6):

$$
c_{0} \in\left\{m \cdot \nu: m \in \mathbb{Z}^{k}\right\} .
$$

Thus $n \in \mathbb{Z}^{k}$ exists such that $c_{0}=n \cdot \nu$. Clearly $n$ is unique if $\nu$ is nonresonant.

We can now prove Theorem 5.3.

Proof of Theorem 5.3: Consider $U=:\left[u^{1}, u^{2}, u^{3}\right]$ and $\hat{U}=:\left[\hat{u}^{1}, \hat{u}^{2}, \hat{u}^{3}\right]$, both in $\mathfrak{U}\left(\phi_{0}\right)$, and define the smooth functions $g_{ \pm}: \mathbb{R} \rightarrow \mathbb{C}$ by

$$
g_{ \pm}:=\left(u^{1} \pm i u^{2}\right) \cdot\left(\hat{u}^{1}+i \hat{u}^{2}\right) .
$$

It follows from (2.17) that

$$
\dot{g}_{ \pm}=i\left(\nu_{s}(\hat{U}) \pm \nu_{s}(U)\right) g_{ \pm}
$$

so that

$$
g_{ \pm}(\theta)=\exp \left\{i\left(\nu_{s}(\hat{U}) \pm \nu_{s}(U)\right) \theta\right\} g_{ \pm}(0) .
$$

Because $U$ and $\hat{U}$ are in $\mathcal{Q}(1, \omega ; d+1),(5.8)$ ensures that $g_{ \pm}$are also in $\mathcal{Q}(1, \omega ; d+1)$. Now, if $g_{+}$is not the zero function then by (5.9) $\exp \left\{i\left(\nu_{s}(\hat{U})+\nu_{s}(U)\right) \theta\right\}$ is in $\mathcal{Q}(1, \omega ; d+1)$ and by Lemma $5.6 \nu_{s}(\hat{U})+\nu_{s}(U)=m$. $(1, \omega)$ for some $m \in \mathbb{Z}^{d+1}$. Thus either $g_{+}=0$ or $m \in$ $\mathbb{Z}^{d+1}$ exist such that $\nu_{s}(\hat{U})+\nu_{s}(U)=m \cdot(1, \omega)$. Similarly either $g_{-}=0$ or $m \in \mathbb{Z}^{d+1}$ exist such that 
$\nu_{s}(\hat{U})-\nu_{s}(U)=m \cdot(1, \omega)$. By definition either $g_{+}$or $g_{-}$ is different from the zero function, since otherwise $u^{j}$. $\hat{u}^{k}=0$ for $(j, k=1,2)$, which is obviously false. Thus $m \in \mathbb{Z}^{d+1}$ exist such that either $\nu_{s}(\hat{U})+\nu_{s}(U)=$ $m \cdot(1, \omega)$ or $\nu_{s}(\hat{U})-\nu_{s}(U)=m \cdot(1, \omega)$. Hence $\varepsilon \in$ $\{-1,1\}$ exists such that $\nu_{s}(\hat{U})=\varepsilon \nu_{s}(U)+m \cdot(1, \omega)$, i.e., $\Xi\left(\phi_{0}\right) \subset\left[\nu_{s}(U)\right]$. But by the remarks before Theorem 5.3 we also have that $\Xi\left(\phi_{0}\right) \supset\left[\nu_{s}(U)\right]$.

\section{Remarks}

(1) For the case of $J=0$ and arbitrary $\phi_{0}$, each Floquet frequency $\nu_{0}$ is in $\Xi\left(\phi_{0}\right)$ (see Remark 2 in Sec. III). We will see in Remark 4 of Sec. VI that the torus $J=0$ is well-tuned so that every Floquet frequency $\nu_{0}$ is a spin tune.

(2) As we will see in Remarks 13 and 14 in Sec. VI, matrices $\mathcal{A}(\theta, \phi)$ can be found with which there are tori where $\Xi\left(\phi_{0}\right) \neq \emptyset$ for every $\phi_{0}$ but which are not welltuned.

(3) Consider a well-tuned torus. We say that the torus is on a "spin-orbit resonance" if $0 \in \Xi$. Thus on spin-orbit resonance the set of spin tunes reads as $\Xi=\left\{\omega_{s} \in\right.$ $\left.[0,1): \omega_{s}=j_{0}+j \cdot \omega ; j_{0} \in \mathbb{Z}, j \in \mathbb{Z}^{d}\right\}$. For $d=3$ the spin-orbit resonance condition amounts to (1.3). In general the condition takes the form

$$
\omega_{s}=m_{0}+m_{1} \omega_{1}+\cdots+m_{d} \omega_{d},
$$

and the order of the resonance is $\left|m_{1}\right|+\cdots+\left|m_{d}\right|$. Thus on a well-tuned torus, if one $\phi_{0}$ has resonant spin motion, then all $\phi_{0}$ have resonant spin motion. Of course the same is true for nonresonant spin motion. This is a key aspect of being well-tuned: spin-orbit resonance is not defined in terms of a spin frequency $\omega_{s}$ that varies with $\phi_{0}$ in the sense that $\left[\omega_{s}\right]$ is dependent on $\phi_{0}$. An important consequence of this definition of resonance is presented in Theorem 6.4. Clearly the order of a resonance depends on the chosen $\omega_{s}$ and that in turn depends on the chosen UPF. Thus the order of a spin-orbit resonance is frame dependent. However, in practice the order is fixed by choosing a "preferred" spin tune and the corresponding UPF for every $J$. The way to do this will be explained in Sec. X, where some numerical results on spin-orbit resonance are also mentioned. A numerical method for computing $\Xi$ is outlined in Sec. IX.

(4) If one component of $\omega$ is irrational and if the torus is well-tuned, then from the previous discussion the sets $\Xi$ and $[0]$ are countable dense subsets of $[0,1)$. Thus even though the complement of $\Xi$ is uncountable and a number chosen at random from $[0,1)$ is unlikely to be a spin tune, $\Xi$ contains spin tunes arbitrarily close to any number in this interval. This simply implies that every spin tune (in particular the preferred spin tune) is close to a number in the resonance set [0] so that the system is always arbitrarily near to a high order resonance. Although the set $[0]$ is a dense subset of $[0,1)$, the subset of low order spinorbit resonances is not dense in $[0,1)$. Thus the closeness of a preferred spin tune $\omega_{s}$ to a number in the set [0] does not mean that $\omega_{s}$ is near to a low order spin-orbit resonance.

(5) The set $\Xi_{J}$ of proper UPRs on the torus $J$ is a function of $J$, which is uniquely determined by the $J$ dependence of $\mathcal{A}$ and the orbital tune $\omega$. Of course, if in addition, $\mathcal{A}$ depends on extra parameters like the beam energy, then the set $\Xi_{J}$ is also a function of these parameters. Thus if all tori are well-tuned the spin tunes $\omega_{s}$ will vary with $J$ and any extra parameters. Therefore the distance $\left|\omega_{s}-m_{0}-m_{1} \omega_{1}-\cdots-m_{d} \omega_{d}\right|$ from the spin-orbit resonance condition (5.10) depends on $J$ and any extra parameters.

(6) Consider a torus for which $\mathfrak{U}\left(\phi_{0}\right) \neq \emptyset$ for each $\phi_{0}$, and for each $\phi_{0}$ choose a single proper $\operatorname{UPF} U\left(\cdot ; \phi_{0}\right)$. The corresponding UPRs $\nu_{s}\left(U\left(\cdot ; \phi_{0}\right)\right)$ define a function of $\phi_{0}$. If the torus is well-tuned, then $\nu_{s}\left(U\left(\cdot ; \phi_{0}\right)\right)$ can be chosen to be a constant. However for an ill-tuned torus, $\nu_{s}\left(U\left(\cdot ; \phi_{0}\right)\right)$ usually has to vary with $\phi_{0}$. This nonconstant function can nevertheless be continuous and we present examples in Remarks 13 and 14 in Sec. VI. However, because of the freedom to choose the proper UPRs, $\nu_{s}\left(U\left(\cdot ; \phi_{0}\right)\right)$, this function is usually very irregular and discontinuous.

(7) If a torus is ill-tuned, then in domains of $\phi_{0}$ where $\nu_{s}\left(U\left(\cdot ; \phi_{0}\right)\right)$ varies with $\phi_{0}$, it is likely that there are some $\phi_{0}$ 's for which the resonance condition $\nu_{s}\left(U\left(\cdot ; \phi_{0}\right)\right)=$ $m_{0}+m \cdot \omega$ holds.

(8) If the principal solution matrix $\Phi$ can be written as

$$
\Phi\left(\theta ; \phi_{0}\right)=p\left(\theta ; \phi_{0}\right) \exp \left(B\left(\phi_{0}\right) \nu\left(\phi_{0}\right) \theta\right),
$$

where $\nu\left(\phi_{0}\right) \in[0,1), B\left(\phi_{0}\right)$ is a real skew-symmetric matrix with spectrum $\lambda\left(B\left(\phi_{0}\right)\right)=\{i,-i, 0\}$ and the matrix $p\left(\cdot ; \phi_{0}\right)$ is in $Q(1, \omega ; d+1)$, then $p, B, \nu$ will be called generalized Floquet parameters at $\phi_{0}$. In particular $\nu\left(\phi_{0}\right)$ will be called a generalized Floquet frequency. By definition the generalized Floquet parameter $p\left(\theta ; \phi_{0}\right)$ is smooth in $\theta$ and $p \in$ $\mathrm{SO}(3)$, with $p\left(0 ; \phi_{0}\right)=I$. Because $p\left(\cdot ; \phi_{0}\right)$ has the same periodicities as $A\left(\cdot ; \phi_{0}\right)$, the $p, B, \nu$ are indeed generalizations of the Floquet parameters introduced in Sec. III. Note that (5.11) generates a large class of $A$ 's, namely, $A\left(\theta ; \phi_{0}\right)=(\partial \Phi / \partial \theta)\left(\theta ; \phi_{0}\right) \Phi^{T}\left(\theta ; \phi_{0}\right)$, where $\Phi$ is given by (5.11).

If $p\left(\theta ; \phi_{0}\right), B\left(\phi_{0}\right), \nu\left(\phi_{0}\right)$ are generalized Floquet parameters at $\phi_{0}$, then from Lemma 2.1(b) a $W\left(\phi_{0}\right) \in$ $\mathrm{SO}(3)$ exists such that $W\left(\phi_{0}\right) J W^{T}\left(\phi_{0}\right)=B\left(\phi_{0}\right)$, whence $\Phi\left(\theta ; \phi_{0}\right)=p\left(\theta ; \phi_{0}\right) W\left(\phi_{0}\right) e^{J \nu\left(\phi_{0}\right) \theta}\left(p\left(0 ; \phi_{0}\right) W\left(\phi_{0}\right)\right)^{T}$. Thus $p\left(\theta ; \phi_{0}\right) W\left(\phi_{0}\right)$ is a proper UPF at $\phi_{0}$ with UPR $\nu\left(\phi_{0}\right)$.

Conversely, if $U\left(\theta ; \phi_{0}\right)$ is a proper UPF at $\phi_{0}$, then by (2.15), $\Phi$ can be written as in (5.11) with $p, B, \nu$ given by

$$
\begin{aligned}
p\left(\theta ; \phi_{0}\right) & :=U\left(\theta ; \phi_{0}\right) U^{T}\left(0 ; \phi_{0}\right), \\
B\left(\phi_{0}\right) & :=U\left(0 ; \phi_{0}\right) J U^{T}\left(0 ; \phi_{0}\right), \\
\nu\left(\phi_{0}\right) & :=\nu_{s}\left(U\left(\cdot ; \phi_{0}\right)\right) .
\end{aligned}
$$


Therefore the UPR corresponding to a proper UPF is a generalized Floquet frequency and is thus a frequency additional to those in $A$ in analogy with the Floquet frequency which emerges in the case where $A$ is $2 \pi$-periodic. We conclude that the set of generalized Floquet frequencies at an arbitrary $\phi_{0}$ is identical with the set $\Xi\left(\phi_{0}\right)$.

Note that the condition $\Xi\left(\phi_{0}\right) \neq \emptyset$ at some $\phi_{0}$ implies that generalized Floquet parameters exist at that $\phi_{0}$. This can be viewed as a generalized Floquet theorem. Moreover, if the torus is well-tuned, then for every $\phi_{0}$ generalized Floquet parameters exist and the set of generalized Floquet frequencies is the same at every $\phi_{0}$ and is identical with the set $\Xi$ of spin tunes.

(9) The spin trajectories at a given $\phi_{0}$ are quasiperiodic if $\Xi\left(\phi_{0}\right) \neq \emptyset$, but there are $A\left(\cdot ; \phi_{0}\right)$ in $\mathcal{Q}(1, \omega ; d+1)$ which give rise to nonquasiperiodic spin trajectories (see Sec. VIII).

\section{THE INVARIANT SPIN FIELD AND THE QUASIPERIODICITY OF THE SPIN MOTION}

In this section we present an application of the concepts introduced in Sec. V. In particular we consider the important situation where, for a given $\mathcal{A}(\cdot, J)$, a torus admits an invariant spin field, a central object in the theory of polarization whose relevance was explained in the Introduction. It was pointed out there that the invariant spin field is a solution to the T-BMT equation along particle orbits and that it is $2 \pi$-periodic in $\theta$ and $\phi$. We now return to it and show, among other things, that if the torus is off orbital resonance, the existence of a nonunique invariant spin field implies that the system is on spinorbit resonance. We begin by studying general spin fields.

Let $S(\theta, \phi)$ be a field such that $S\left(\theta ; \phi_{0}\right):=S\left(\theta, \phi_{0}+\right.$ $\omega \theta)$ is the spin of the particle which starts with phase $\phi_{0}$ at $\theta=0$. By (2.1) we must have

$$
\begin{aligned}
\frac{d}{d \theta} S\left(\theta ; \phi_{0}\right) & =\mathcal{D} S\left(\theta, \omega \theta+\phi_{0}\right) \\
& =\mathcal{A}\left(\theta, \omega \theta+\phi_{0}\right) S\left(\theta, \omega \theta+\phi_{0}\right),
\end{aligned}
$$

and thus the evolution of the field is described by the partial differential equation

$$
\star \star \star \quad \mathcal{D} S=\mathcal{A}(\theta, \phi) S \text {. }
$$

Here, $\mathcal{D} S(\theta, \phi)=D_{1} S(\theta, \phi)+\omega \cdot \nabla_{\phi} S(\theta, \phi)$, where $D_{k}$ will denote the derivative with respect to the $k$ th argument, be it scalar or multicomponent. Consider the generalized principal solution matrix $\varphi(\theta, \phi)$ defined by

$$
\mathcal{D} \varphi=\mathcal{A}(\theta, \phi) \varphi, \quad \varphi(0, \phi)=I,
$$

then the general solution of (6.1) is given by

$$
S(\theta, \phi)=\varphi(\theta, \phi) S(0, \phi-\omega \theta)
$$

This can be seen directly: since $\mathcal{D}$ is a derivation,
$\mathcal{D} S(\theta, \phi)=(\mathcal{D} \varphi(\theta, \phi)) S(0, \phi-\omega \theta)+\varphi(\theta, \phi) \mathcal{D}(S(0, \phi-$ $\omega \theta)$ ) and $\mathcal{D}(S(0, \phi-\omega \theta))=0$. Equations (6.1) and (6.2) are amenable to solution by the method of characteristics. In this case the characteristics for (6.1) are just the spin trajectories $S\left(\cdot, \phi_{0}\right)$ and the characteristics for (6.2) are just the $\Phi\left(\cdot, \phi_{0}\right)$.

Now suppose, as in Sec. II, that we know one solution of (6.1). Let $\mathcal{V}(\theta, \phi)$ be in $\operatorname{SO}(3)$ such that its third column is this solution and make the transformation $\varphi \rightarrow$ $\psi$ defined by

$$
\varphi=\mathcal{V} \psi
$$

Then

$$
\mathcal{D} \varphi=(\mathcal{D} \mathcal{V}) \psi+\mathcal{V} \mathcal{D} \psi=\mathcal{A} \mathcal{V} \psi
$$

so that

$$
\mathcal{D} \psi=C_{\mathcal{V}}(\theta, \phi) J \psi, \quad \psi(0, \phi)=\mathcal{V}^{T}(0, \phi),
$$

where, in analogy to Sec. II, $C_{\mathcal{V}}$ is defined by

$$
\mathcal{V}^{T}(\mathcal{A} \mathcal{V}-\mathcal{D} \mathcal{V})=C_{\mathcal{V}} J
$$

and thus

$$
\begin{gathered}
C_{\mathcal{V}}=-(1 / 2) \operatorname{Tr}\left[J\left(\mathcal{V}^{T} \mathcal{A} \mathcal{V}-\mathcal{V}^{T} \mathcal{D} \mathcal{V}\right)\right] \\
\mathcal{D} \mathcal{V}=\mathcal{A} \mathcal{V}-C_{\mathcal{V}} \mathcal{V} J
\end{gathered}
$$

which is analogous to (2.13).

Equation (6.5) also can be solved by the method of characteristics giving

$$
\begin{aligned}
& \psi(\theta, \phi) \\
& =\exp \left(J \int_{0}^{\theta} C_{\mathcal{V}}\left(\theta^{\prime}, \omega \theta^{\prime}+\phi-\omega \theta\right) d \theta^{\prime}\right) \mathcal{V}^{T}(0, \phi-\omega \theta),
\end{aligned}
$$

as is easily checked by direct substitution in (6.5). The generalized principal solution matrix thus becomes

$$
\begin{aligned}
& \varphi(\theta, \phi) \\
&= \mathcal{V}(\theta, \phi) \exp \left(J \int_{0}^{\theta} C_{\mathcal{V}}\left(\theta^{\prime}, \omega \theta^{\prime}+\phi-\omega \theta\right) d \theta^{\prime}\right) \\
& \times \mathcal{V}^{T}(0, \phi-\omega \theta) .
\end{aligned}
$$

The principal solution matrix of (5.1) is easily constructed from the generalized principal solution matrix as the following theorem shows.

Theorem 6.1 Let $\mathcal{V}(\theta, \phi)$ be a smooth $\mathrm{SO}(3)$ matrix on a fixed torus, with third column satisfying (6.1). Then the 
principal solution matrix at $\phi_{0}$ on the torus is given by

$$
\begin{aligned}
\star \star \star \Phi & \left(\theta ; \phi_{0}\right) \\
= & \varphi\left(\theta, \omega \theta+\phi_{0}\right) \\
= & \mathcal{V}\left(\theta, \omega \theta+\phi_{0}\right) \exp \left(J \int_{0}^{\theta} C_{\mathcal{V}}\left(\theta^{\prime}, \omega \theta^{\prime}+\phi_{0}\right) d \theta^{\prime}\right) \\
& \times \mathcal{V}^{T}\left(0, \phi_{0}\right) .
\end{aligned}
$$

Proof: Clearly $\Phi\left(0 ; \phi_{0}\right)=I$. Moreover, $\Phi$ satisfies (5.1) because $(d / d \theta) \mathcal{V}\left(\theta, \omega \theta+\phi_{0}\right)=\mathcal{A} \mathcal{V}-C_{\mathcal{V}} \mathcal{V} J$ and $(d / d \theta) \int_{0}^{\theta} C_{\mathcal{V}}\left(\theta^{\prime}, \omega \theta^{\prime}+\phi_{0}\right) d \theta^{\prime}=C_{\mathcal{V}}\left(\theta, \omega \theta+\phi_{0}\right)$.

We now give several definitions. In particular, we give a formal definition of the invariant spin field.

Definition 6.2 (ISF, IFF): Consider a fixed torus.

(a) A field $S(\theta, \phi) \in \mathbb{R}^{3}$ is said to be a spin field for (2.1) if and only if $S$ is smooth in $(\theta, \phi), 2 \pi$-periodic in $\phi$ and if it satisfies the partial differential equation (6.1). A spin field $S$ is called an invariant spin field (ISF) for (2.1) if it is also $2 \pi$-periodic in $\theta$ and $|S|=1$.

(b) $\mathrm{An} \operatorname{SO}(3)$ matrix, $\mathcal{V}(\theta, \phi)$, is called a frame field for (2.1) if and only if $\mathcal{V}$ is smooth in $(\theta, \phi), 2 \pi$-periodic in $\phi$ and if its third column is a spin field. A frame field is called an invariant frame field (IFF) for (2.1) if and only if it is also $2 \pi$-periodic in $\theta$. Thus the third column of an IFF is an ISF. A uniform IFF $\mathcal{V}$ is such that the function $C_{\mathcal{V}}$ as defined by (6.7) is constant [independent of $(\theta, \phi)$ ] and in $[0,1)$.

Note that in (6.10) both $\mathcal{V}$ and $\varphi$ are frame fields.

Theorem 6.3 (a) Consider a fixed torus. If a uniform IFF $\mathcal{V}$ exists, then, for every $\phi_{0}, U\left(\cdot ; \phi_{0}\right)$, defined by $U\left(\theta ; \phi_{0}\right):=\mathcal{V}\left(\theta, \phi_{0}+\omega \theta\right)$, is a proper UPF at $\phi_{0}$ with $\nu_{s}\left(U\left(\cdot ; \phi_{0}\right)\right)=C_{V}$. Moreover, the torus is welltuned and $C_{\mathcal{V}}$ is a spin tune.

(b) Consider a fixed torus. If $\mathcal{V}$ is a frame field then it satisfies the partial differential equation (6.8), where $C_{\mathcal{V}}$ is given by (6.7).

Proof of Theorem 6.3(a): From Theorem 6.1 it follows that $U\left(\cdot ; \phi_{0}\right)$ is a UPF at $\phi_{0}$ with UPR $\nu_{s}\left(U\left(\cdot ; \phi_{0}\right)\right)=C_{\mathcal{V}}$. Of course, $U\left(\cdot ; \phi_{0}\right)$ is in $\mathcal{Q}(1, \omega ; d+1)$ so that the UPF $U\left(\cdot ; \phi_{0}\right)$ is proper. Because $C_{\mathcal{V}}$ is contained in each $\Xi\left(\phi_{0}\right)$ we conclude (recall the comment after Definition 5.4) that the torus is well-tuned and that $C_{\mathcal{V}}$ is a spin tune.

Proof of Theorem 6.3b: With

$$
\mathcal{V}=:\left[\mathfrak{v}^{1}, \mathfrak{v}^{2}, \mathfrak{v}^{3}\right]
$$

and

$$
\mathfrak{w}^{j}:=(\mathcal{D}-\mathcal{A}) \mathfrak{b}^{j},
$$

and since $\mathcal{V}$ is in $\operatorname{SO}(3)$ and $S \equiv \mathfrak{v}^{3}$ satisfies (6.1), we have

$$
\mathfrak{w}^{1}=\left(\mathfrak{w}^{1} \cdot \mathfrak{v}^{2}\right) \mathfrak{v}^{2}, \quad \mathfrak{w}^{2}=-\left(\mathfrak{w}^{1} \cdot \mathfrak{v}^{2}\right) \mathfrak{v}^{1}, \quad \mathfrak{w}^{3}=0,
$$

so that

$$
\mathcal{D} \mathcal{V}-\mathcal{A} \mathcal{V}=\left(\mathfrak{w}^{1} \cdot \mathfrak{v}^{2}\right) \mathcal{V} J
$$

Because $\operatorname{Tr}\left[J^{2}\right]=-2$, it follows from (6.14) that $\mathfrak{w}^{1} \cdot \mathfrak{v}^{2}=(1 / 2) \operatorname{Tr}\left[J\left(\mathcal{V}^{T} \mathcal{A} \mathcal{V}-\mathcal{V}^{T} \mathcal{D} \mathcal{V}\right)\right]$

We now make some further remarks on IFFs and ISFs.

\section{Remarks}

(1) The name invariant frame field is chosen so as to reflect the fact that, like the ISF, it is $2 \pi$-periodic in $\theta$.

(2) If $\mathcal{V}$ is an IFF, then we call the $\mathfrak{v}^{1}, \mathfrak{v}^{2}$ in (6.12) pseudo- $u^{1}, u^{2}$-axes. They are used in the program SPRINT for the numerical calculation of the spin tune $[6,8,9,12,13,23,25]$. If the IFF is uniform, then $\mathfrak{v}^{1}, \mathfrak{v}^{2}$ are called $u^{1}, u^{2}$-axes (see also [22,24]). An example of a numerical calculation of $u^{1}, u^{2}$-axes can be found in [13]; see also Sec. X.

(3) From the proof of Theorem 6.3(b) it follows for every IFF $\mathcal{V}$ that (see also [24])

$$
\begin{aligned}
C_{\mathcal{V}}(\theta, \phi) & =\mathfrak{v}^{1} \cdot\left(-\mathcal{A}(\theta, \phi) \mathfrak{v}^{2}+\mathcal{D V}^{2}\right) \\
& =\Omega \cdot \mathfrak{v}^{3}+\mathfrak{v}^{1} \cdot\left(\mathcal{D} \mathfrak{v}^{2}\right) .
\end{aligned}
$$

Thus if $\mathfrak{b}^{2}$ is an ISF, then $C_{\mathcal{V}}=0$ and $\mathcal{V}$ is a uniform IFF.

(4) A uniform IFF always exists for the closed orbit so that by Theorem 6.3(a) the torus $J=0$ is well-tuned. This follows easily from the fact that in this case proper UPFs are uniform IFFs (see Remark 2 in Sec. III). Thus at $J=$ 0 every Floquet frequency is a spin tune by Remark 1 of Sec. V. Note also that for $J=0$ any $2 \pi$-periodic spin trajectory $S$ with $|S|=1$ is an ISF.

(5) Because the coefficients of the partial differential equation (6.1) are $2 \pi$-periodic, it is plausible that an ISF exists. But as we signaled earlier, it remains as a mathematical challenge to prove it, and in Remark 14 we will give an example where no ISF exists. The coefficients of the partial differential equation (6.8) are also $2 \pi$-periodic so that it is again plausible, but again mathematically challenging to prove, that an IFF exists. Note that if an ISF $S$ exists, then an IFF $\mathcal{V}$ is easily constructed in analogy with the construction of $V(\theta)$ in Sec. III if there is a $2 \pi$-periodic smooth unit vector which is nowhere parallel to $S$ (see also [24]). However, if there is no such unit vector then it can happen that an IFF $\mathcal{V}$ with the third column $S$ does not exist.

If an ISF does exist, it might not be unique. In that case the torus is on a spin-orbit resonance, as shown in the following theorem.

Theorem 6.4 Consider the case of a fixed torus which is off orbital resonance and assume that an ISF, $n$, exists. Let $n$ be nonunique, i.e., let $\hat{n}$ be another ISF such that the vector product of $n$ and $\hat{n}$ is nonzero at some $(\theta, \phi)$. Then a uniform IFF exists (so that the torus is well-tuned) and the system is on a spin-orbit resonance.

Proof: Let $n$ and $\hat{n}$ be ISFs whose vector product is nonzero at some $(\theta, \phi)$ and let the torus be off orbital resonance. We observe that $F:=|n \times \hat{n}|^{2}$ is a smooth and 
$2 \pi$-periodic function with $\mathcal{D} F=0$. As will be shown below, it follows that off orbital resonance the function $F$ is constant. Thus the angle between the ISFs is the same at all points and $m$, defined by $m:=(n \times \hat{n}) /|n \times \hat{n}|$, is an ISF, perpendicular to $n$. Therefore $\mathcal{V}:=[m \times n, m, n]$ is an IFF. Due to Remark 3 the IFF $\mathcal{V}$ is uniform with $C_{V}=0$. We conclude from Theorem 6.3(a) that the torus is well-tuned and that 0 is a spin tune. In particular the torus is on a spin-orbit resonance (see also Remark 3 in Sec. V).

To complete the proof, we now consider a smooth and $2 \pi$-periodic function $\mathcal{F}: \mathbb{R}^{d+1} \rightarrow \mathbb{R}$ for which $\mathcal{D} \mathcal{F}=0$. We define $\mathcal{H}: \mathbb{R}^{d+1} \rightarrow \mathbb{R}$ via

$$
\mathcal{H}(\theta, \phi):=\mathcal{F}(\theta, \phi+\omega \theta) .
$$

Hence $\mathcal{H}(\theta, \phi)$ is a smooth function $2 \pi$-periodic in $\phi$ such that $D_{1} \mathcal{H}=0$. Because $\mathcal{H}$ is smooth, we have

$$
\begin{aligned}
\mathcal{H}(\theta, \phi) & =\mathcal{H}(0, \phi)+\int_{0}^{\theta} D_{1} \mathcal{H}\left(\theta_{0}, \phi\right) d \theta_{0} \\
& =\mathcal{H}(0, \phi) .
\end{aligned}
$$

Because $\mathcal{F}$ is $2 \pi$-periodic in $\theta$, we obtain

$$
\begin{aligned}
0 & =\mathcal{F}(2 \pi, \phi)-\mathcal{F}(0, \phi) \\
& =\mathcal{H}(2 \pi, \phi-2 \pi \omega)-\mathcal{H}(0, \phi),
\end{aligned}
$$

whence by (6.17)

$$
0=\mathcal{H}(0, \phi-2 \pi \omega)-\mathcal{H}(0, \phi) .
$$

Thus it follows that for the Fourier coefficients $g_{r}:=(1 / 2 \pi)^{d} \int_{0}^{2 \pi} \cdots \int_{0}^{2 \pi} \mathcal{H}(0, \phi) \exp (-i r \cdot \phi) d \phi \quad$ of $\mathcal{H}(0, \cdot)$

$$
g_{r}=\exp (2 \pi i r \cdot \omega) g_{r}
$$

where $r \in \mathbb{Z}^{d}$. Because $(1, \omega)$ is nonresonant $g_{r}$ vanishes for $r \neq 0$. Thus by Lemma 4.3(a) $\mathcal{H}(0, \phi)$ is constant, i.e., independent of $\phi$. Therefore, by (6.17) $\mathcal{H}(\theta, \phi)$ is constant, i.e., independent of $\theta, \phi$ and then by (6.16) $\mathcal{F}(\theta, \phi)$ is constant, i.e., independent of $\theta, \phi$.

Theorem 6.4 addresses the uniqueness of the ISF as well as its nonuniqueness. In particular, the contrapositive of Theorem 6.4 yields: if off orbital resonance a uniform IFF exists, and if the system is not on spin-orbit resonance, then the ISF is unique up to a sign. This behavior was predicted earlier in [22].

We now focus on the case where an IFF exists and $C_{\mathcal{V}}$ is not constant. We first define

$$
c_{\mathcal{V}}\left(\theta ; \phi_{0}\right):=C_{\mathcal{V}}\left(\theta, \omega \theta+\phi_{0}\right) \text {. }
$$

Since $c_{\mathcal{V}}\left(\cdot ; \phi_{0}\right) \in \mathcal{Q}(1, \omega ; d+1)$, its mean $\bar{c}_{\mathcal{V}}\left(\phi_{0}\right)$ and zero-mean part $\tilde{c} \mathcal{V}\left(\theta ; \phi_{0}\right)$ exist. We thus have an important decomposition of $C_{\mathcal{V}}$, namely,

$$
C_{\mathcal{V}}(\theta, \phi)=\bar{c}_{\mathcal{V}}(\phi-\omega \theta)+\tilde{c}_{\mathcal{V}}(\theta ; \phi-\omega \theta) .
$$

Since the lhs is $2 \pi$-periodic, the rhs is, too, and in fact
$\bar{c}_{\mathcal{V}}(\phi-\omega \theta)$ and $\tilde{c}_{\mathcal{V}}(\theta ; \phi-\omega \theta)$ are individually $2 \pi$-periodic as we check in Lemma 6.6(a) below. From (6.21) the integral in the exponential of (6.10) is

$$
\begin{aligned}
& \int_{0}^{\theta} C_{\mathcal{V}}\left(\theta^{\prime}, \omega \theta^{\prime}+\phi-\omega \theta\right) d \theta^{\prime} \\
& \quad=\theta \bar{c}_{\mathcal{V}}(\phi-\omega \theta)+\beta_{\mathcal{V}}(\theta, \phi),
\end{aligned}
$$

where

$$
\beta_{\mathcal{V}}(\theta, \phi):=\int_{0}^{\theta} \tilde{c}_{\mathcal{V}}\left(\theta^{\prime} ; \phi-\omega \theta\right) d \theta^{\prime} .
$$

Clearly $\mathcal{D} \beta_{\mathcal{V}}(\theta, \phi)=\tilde{c} \mathcal{V}(\theta ; \phi-\omega \theta)$, which leads to consideration of the partial differential equation

$$
\mathcal{D} \alpha(\theta, \phi)=\tilde{c}_{\mathcal{V}}(\theta ; \phi-\omega \theta)=: \check{\mathcal{F}}(\theta, \phi) .
$$

Then for every solution $\alpha$ of (6.24)

$$
\beta_{\mathcal{V}}(\theta, \phi)=\alpha(\theta, \phi)-\alpha(0, \phi-\omega \theta) .
$$

The existence of a $2 \pi$-periodic $\alpha$ will be important below (note that $\breve{\mathcal{F}}$ is always $2 \pi$-periodic).

We now write

$$
\bar{c} \mathcal{V}\left(\phi_{0}\right)=\nu_{\mathcal{V}}\left(\phi_{0}\right)+k_{\mathcal{V}}\left(\phi_{0}\right)
$$

where the integer $k_{\mathcal{V}}\left(\phi_{0}\right)$ is uniquely determined by the condition $\nu_{\mathcal{V}}\left(\phi_{0}\right) \in[0,1)$. The generalized principal solution matrix from (6.10) now becomes

$$
\begin{aligned}
\varphi(\theta, \phi)= & \mathcal{V}(\theta, \phi) \exp \{J(\alpha(\theta, \phi)-\alpha(0, \phi-\omega \theta) \\
& \left.\left.+k_{\mathcal{V}}(\phi-\omega \theta) \theta+\nu_{\mathcal{V}}(\phi-\omega \theta) \theta\right)\right\} \\
& \times \mathcal{V}^{T}(0, \phi-\omega \theta) \\
= & \mathcal{U}(\theta, \phi) \exp \left(J \nu_{\mathcal{V}}(\phi-\omega \theta) \theta\right) \mathcal{U}^{T}(0, \phi-\omega \theta),
\end{aligned}
$$

where

$$
\mathcal{U}(\theta, \phi):=\mathcal{V}(\theta, \phi) \exp \left\{J\left(\alpha(\theta, \phi)+k_{\mathcal{V}}(\phi-\omega \theta) \theta\right)\right\}
$$

Then the principal solution matrix becomes

$$
\Phi\left(\theta ; \phi_{0}\right)=U\left(\theta ; \phi_{0}\right) \exp \left(J \nu_{\mathcal{V}}\left(\phi_{0}\right) \theta\right) U^{T}\left(0 ; \phi_{0}\right)
$$

where

$$
\begin{aligned}
& U\left(\theta ; \phi_{0}\right) \\
& \quad:=\mathcal{U}\left(\theta, \omega \theta+\phi_{0}\right) \\
& \quad=\mathcal{V}\left(\theta, \omega \theta+\phi_{0}\right) \exp \left\{J\left(\alpha\left(\theta, \omega \theta+\phi_{0}\right)+k_{\mathcal{V}}\left(\phi_{0}\right) \theta\right)\right\}
\end{aligned}
$$

We can now state and prove the next basic result of this section. The proof will depend, in part, on Lemma 6.6(b), which follows later in order not to break the flow. 
Theorems 6.5(c) and 6.5(d) use the Diophantine condition.

Theorem 6.5 Consider a fixed torus $J_{0}$.

(a) If an IFF $\mathcal{V}$ exists and (6.24) has a smooth and $2 \pi$-periodic solution $\alpha$, then $U\left(\cdot ; \phi_{0}\right)$, defined by (6.30), is a proper UPF at $\phi_{0}$ with UPR equal to $\nu_{\mathcal{V}}\left(\phi_{0}\right)$.

(b) If the conditions of Theorem 6.5(a) hold and if the torus is off orbital resonance, then $\mathcal{U}$, defined by (6.28), is a uniform IFF and $C_{u}=\nu_{\mathcal{V}}$. Moreover, the torus is well-tuned and $U\left(\cdot ; \phi_{0}\right)$, defined for every $\phi_{0}$ by $(6.30)$, is a proper UPF at $\phi_{0}$ whose UPR is a spin tune and $\nu_{s}\left(U\left(\cdot ; \phi_{0}\right)\right)=C_{u}=\nu_{\mathcal{V}}$.

(c) Let $0<\tau<r-d-3$ and let $\mathcal{A}\left(\cdot, J_{0}\right)$ be in $C^{r}$. If an IFF $\mathcal{V}$ exists in $C^{r}$ and if $\omega\left(J_{0}\right) \in \Omega(\tau)$, then $a$ uniform IFF exists (and thus the torus is well-tuned).

(d) Let $r>2 d+4$ and let $\mathcal{A}\left(\cdot, J_{0}\right)$ be in $C^{r}$. If an IFF $\mathcal{V}$ exists in $C^{r}$ for every $\omega\left(J_{0}\right)$ in a Borel subset $\mathcal{R}$ of $\mathbb{R}^{d}$, then a uniform IFF exists (and thus the torus is welltuned) for $\mu$-almost every $\omega\left(J_{0}\right)$ in $\mathcal{R}$.

Proof of Theorem 6.5(a): It is clear from (6.30) that $U\left(\cdot ; \phi_{0}\right)$ is in $Q(1, \omega ; d+1)$ and the result follows from (6.29).

Proof of Theorem 6.5(b): From (6.28), $\mathcal{U}$ and $\mathcal{V}$ have the same third column and $U$ is smooth and $2 \pi$-periodic. Therefore $\mathcal{U}$ is an IFF. From (6.7) an easy calculation gives $C_{u}=\nu_{\mathcal{V}}$. Thus by Lemma 6.6(b), $C_{U}$ is constant, independent of $\phi_{0}$. Hence, $\mathcal{U}$ is a uniform IFF. Therefore, by Theorem 6.3(a) $U\left(\cdot ; \phi_{0}\right)$ is a proper UPF at $\phi_{0}$ with UPR $\nu_{\mathcal{V}}$ which is a spin tune and the torus is well-tuned.
Proof of Theorem 6.5(c): From (6.7), $C_{\mathcal{V}}$ is in $C^{r-1}$ since $\mathcal{V}$ and $\mathcal{A}$ are in $C^{r}$. Using the fact that the torus is off orbital resonance [since $\omega(J) \in \Omega(\tau)$ ], we have by Lemma 6.6(b) that $\bar{c} \mathcal{V}$ is a constant. Thus, by (6.21), $\breve{\mathcal{F}}=$ $C_{\mathcal{V}}-\bar{c}_{\mathcal{V}}$ is in $C^{r-1}$. It follows from the condition $\tau<$ $r-d-3$ and Lemma 4.7 that

$$
\alpha(\theta, \phi)=\sum_{m \in \mathbb{Z}^{d+1} \backslash\{0\}} \frac{1}{i m \cdot(1, \omega)} \breve{\mathcal{F}}_{m} \exp (i m \cdot(\theta, \phi))
$$

is smooth and $2 \pi$-periodic in $\theta$ and $\phi$ and that it satisfies (6.24), where $\breve{\mathcal{F}}_{m}$ denotes the $m$ th Fourier coefficient of $\check{\mathcal{F}}$. The claims now follow from Theorems $6.5(\mathrm{~b})$ and 6.3(a).

Proof of Theorem 6.5(d): The interval $(d+1, r-d-3)$ is not empty so that we pick a $\tau$ in that interval. Because $\tau<r-d-3$ we have, by Theorem $6.5(\mathrm{c})$, for $\omega\left(J_{0}\right) \in$ $(\Omega(\tau) \cap \mathcal{R})$ a uniform IFF (and thus a well-tuned torus). Because $\tau>d+1$ we have by Lemma 4.8 that $\mu$-almost every $\omega\left(J_{0}\right)$ in $\mathcal{R}$ is in $\Omega(\tau) \cap \mathcal{R}$. This proves our claim.

We now complete the discussion by stating and proving the lemmas promised after (6.21) and before Theorem 6.5.

Lemma 6.6 (a) If $\mathcal{V}$ denotes an IFF, then $\bar{c} \mathcal{V}(\phi-\omega \theta)$ and $\tilde{c} \mathcal{V}(\theta ; \phi-\omega \theta)$ are $2 \pi$-periodic in $\theta$ and in $\phi$.

(b) If $\mathcal{V}$ denotes an IFF and if the torus is off orbital resonance, then $\bar{c}_{\mathcal{V}}, \nu_{\mathcal{V}}$, and $k_{\mathcal{V}}$ are constant.

Proof of Lemma 6.6(a): The periodicities in (6.21) can be demonstrated as follows:

$$
\begin{aligned}
\bar{c} \mathcal{V}(\phi-\omega(\theta+2 \pi)) & =\lim _{T \rightarrow \infty} \frac{1}{T} \int_{0}^{T} C_{\mathcal{V}}\left(\theta^{\prime}, \phi-\omega(\theta+2 \pi)+\omega \theta^{\prime}\right) d \theta^{\prime} \\
& =\lim _{T \rightarrow \infty} \frac{1}{T} \int_{0}^{T} C_{\mathcal{V}}\left(\theta^{\prime}-2 \pi, \phi-\omega(\theta+2 \pi)+\omega \theta^{\prime}\right) d \theta^{\prime} \\
& =\lim _{T \rightarrow \infty} \frac{1}{T} \int_{-2 \pi}^{T-2 \pi} C_{\mathcal{V}}\left(\theta^{\prime \prime}, \phi-\omega \theta+\omega \theta^{\prime \prime}\right) d \theta^{\prime \prime} \\
& =\bar{c}_{\mathcal{V}}(\phi-\omega \theta)+\lim _{T \rightarrow \infty} \frac{1}{T}\left(\int_{-2 \pi}^{0}-\int_{T-2 \pi}^{T}\right) C_{\mathcal{V}}\left(\theta^{\prime \prime}, \phi-\omega \theta+\omega \theta^{\prime \prime}\right) d \theta^{\prime \prime}=\bar{c}_{\mathcal{V}}(\phi-\omega \theta)
\end{aligned}
$$

where at the second equality we have used the fact that $C_{\mathcal{V}}(\cdot, \phi)$ is $2 \pi$-periodic and at the last equality we have used the fact that $C_{\mathcal{V}}$ is bounded. This shows that the first term on the rhs of (6.21) is $2 \pi$-periodic in $\theta$ and thus that all three terms in (6.21) have this periodicity property. That all three terms in (6.21) are $2 \pi$-periodic in $\phi$ is trivial.

Proof of Lemma 6.6( $b$ ): Off orbital resonance we find, due to (6.20) and by applying Lemma 4.3(c), $\bar{c} \mathcal{V}\left(\phi_{0}\right)=$ $\bar{c}_{\mathcal{V}}(0)=(1 / 2 \pi)^{(d+1)} \int_{0}^{2 \pi} \cdots \int_{0}^{2 \pi} C_{\mathcal{V}} d \theta d \phi . \quad$ Thus $\bar{c}_{\mathcal{V}}, \nu_{\mathcal{V}}$, and $k_{\mathcal{V}}$ in (6.26) are $\phi_{0}$ independent in this case.

\section{Remarks}

(6) The $2 \pi$-periodicity in $\theta$ and $\phi$ of $\bar{c} \mathcal{V}(\phi-\omega \theta)$ and $\tilde{c} \mathcal{V}(\theta ; \phi-\omega \theta)$ is suggested by examining the formal
Fourier series $\sum_{m, n} c_{m, n} e^{i(n \theta+m \cdot \phi)}$ of $C_{\mathcal{V}}$ where $n \in$ $\mathbb{Z}, m \in \mathbb{Z}^{d}$. Then it is easy to show that the resonant module part of this sum, defined by $m \cdot(1, \omega)=0$ for $m \in \mathbb{Z}^{d+1}$, corresponds to $\bar{c} \mathcal{V}(\phi-\omega \theta)$. The remaining part with $m \cdot(1, \omega) \neq 0$ corresponds to $\tilde{c}_{\mathcal{V}}(\theta ; \phi-\omega \theta)$. Their formal Fourier series display the $2 \pi$-periodicity in $\theta$ and $\phi$. Off orbital resonance the relation $n+m \cdot \omega=0$ implies that $n=m=0$ so that $\bar{c}_{\mathcal{V}}\left(\phi_{0}\right)=c_{0,0}$, i.e., it is independent of $\phi_{0}$ as in Lemma 6.6(b).

(7) Under the conditions of Theorem 6.5(a), $\nu_{\mathcal{V}}\left(\phi_{0}\right)$ is the UPR at $\phi_{0}$ associated with the UPF defined in (6.30). However, the UPF is not unique since the principal solution matrix in (6.29) can be written as 


$$
\begin{aligned}
\Phi\left(\theta ; \phi_{0}\right)= & U\left(\theta ; \phi_{0}\right) \exp \left(J h\left(\phi_{0}\right)\right) \exp \left(J \nu \mathcal{V}\left(\phi_{0}\right) \theta\right) \\
& \cdot \exp \left(-J h\left(\phi_{0}\right)\right) U^{T}\left(0 ; \phi_{0}\right)
\end{aligned}
$$

so that $\hat{U}\left(\theta ; \phi_{0}\right)=U\left(\theta ; \phi_{0}\right) \exp \left(J h\left(\phi_{0}\right)\right)$ is also a UPF for an arbitrary smooth $h\left(\phi_{0}\right)$.

(8) Under the conditions of Theorem 6.5(a), and using the notation in (6.12), $S\left(\theta ; \phi_{0}\right)=\Phi\left(\theta ; \phi_{0}\right) \mathfrak{v}^{3}\left(0, \phi_{0}\right)$ gives a solution of (2.1) which is in $\mathcal{Q}(1, \omega ; d+1)$. This is easy to check by (6.29): since $\mathcal{V}^{T}\left(0, \phi_{0}\right) \mathfrak{y}^{3}\left(0, \phi_{0}\right)=(0,0,1)$ is the eigenvector of $J$ with zero eigenvalue, $S\left(\theta ; \phi_{0}\right)=$ $\mathfrak{v}^{3}(\theta, \phi(\theta))$ and $\nu_{\mathcal{V}}\left(\phi_{0}\right)$ has dropped out. By (6.29) all other linearly independent solutions are in $\mathcal{Q}\left(1, \omega, \nu_{\mathcal{V}}\left(\phi_{0}\right) ; d+2\right)$.

(9) The IFF underlying the definition (6.26) of $\nu_{\mathcal{V}}\left(\phi_{0}\right)$ is, of course, not unique. For example, if $\nu_{\mathcal{V}}\left(\phi_{0}\right) \in\left(\frac{1}{2}, 1\right)$, then by changing the signs of $\mathfrak{v}^{2}$ and $\mathfrak{v}^{3}$ we find $\nu_{\mathcal{V}}\left(\phi_{0}\right) \in$ $\left(0, \frac{1}{2}\right)$. Thus, in analogy to the case $J=0$ in Remark 7 in Sec. III, we can choose $\mathcal{V}$ such that $\nu_{\mathcal{V}} \in\left[0, \frac{1}{2}\right]$.

(10) If $\mathcal{V}$ denotes a uniform IFF, then $c_{\mathcal{V}}, \bar{c} \mathcal{V}, \nu_{\mathcal{V}}, k_{\mathcal{V}}$ are constant and $\tilde{c}_{\mathcal{V}}$ is zero. In particular, $\nu_{\mathcal{V}}=C_{\mathcal{V}}$.

(11) Under the conditions of Theorem 6.5(b) it is possible, by rotating an arbitrary IFF into a uniform IFF, to construct a proper UPF at every point $\phi_{0}$ of the torus such that the UPR is independent of $\phi_{0}$ and is a spin tune.

(12) Theorem 6.5(a) holds on and off orbital resonance although if $\omega$ does not satisfy a Diophantine condition, appropriate solutions of (6.24) may not exist. Note also that in Sec. VIII we will consider an example where the conditions of Theorem 6.1 hold but where it turns out that the conditions of Theorem 6.5(a) cannot hold due to the presence of nonquasiperiodic spin motion.

(13) To illustrate Theorem 6.5, it is instructive to consider simple, but perhaps unphysical, models. Here we will use the model defined by $\mathcal{A}(\theta, \phi):=\sqrt{2 J} \cos (\phi-$ $\theta) J$ with $d=1$. This represents a precession around the vertical and, of course, such an $\mathcal{A}$ will not be found in real storage rings. Note that $\mathcal{A}$ is a smooth and $2 \pi$-periodic function and, because $(0,0,1)$ is an ISF, we can choose the IFF $\mathcal{V}$ as $\mathcal{V}=I$ so that by (6.7) $C_{\mathcal{V}}(\theta, \phi)=\sqrt{2 J} \cos (\phi-\theta)$. We first consider a case on orbital resonance, $\omega=1$. From $(6.20), c_{\mathcal{V}}\left(\theta ; \phi_{0}\right)=$ $\sqrt{2 J} \cos \left(\phi_{0}\right)$, whence $\bar{c} \mathcal{V}\left(\phi_{0}\right)=c_{\mathcal{V}}\left(\theta ; \phi_{0}\right), \tilde{c}_{\mathcal{V}}=0$. Clearly (6.24) is solved with $\alpha=0$, and (6.30) gives $U\left(\theta ; \phi_{0}\right)=\exp \left(J k_{\mathcal{V}}\left(\phi_{0}\right) \theta\right)$. This $U\left(\cdot ; \phi_{0}\right)$ is a proper UPF at $\phi_{0}$ with UPR $\nu_{\mathcal{V}}\left(\phi_{0}\right)$, where $\nu_{\mathcal{V}}\left(\phi_{0}\right)$ and the integer $k_{\mathcal{V}}\left(\phi_{0}\right)$ are determined by the condition

$$
k_{\mathcal{V}}\left(\phi_{0}\right)+\nu_{\mathcal{V}}\left(\phi_{0}\right)=\sqrt{2 J} \cos \left(\phi_{0}\right) .
$$

Obviously, if $J>0, \Xi$ has uncountably many elements so that the torus is ill-tuned; in particular, by Theorem 6.3(a), no uniform IFF exists.

We now consider the case of irrational $\omega$. From (6.20), $c_{\mathcal{V}}\left(\theta ; \phi_{0}\right)=\sqrt{2 J} \cos \left((\omega-1) \theta+\phi_{0}\right)$, thus $\bar{c}_{\mathcal{V}}=0$ and $\tilde{c} \mathcal{V}\left(\theta ; \phi_{0}\right)=c_{\mathcal{V}}\left(\theta ; \phi_{0}\right)$. Since $\bar{c} \mathcal{V}=0, \nu_{\mathcal{V}}$ and $k_{\mathcal{V}}$ are zero, and since $\mathcal{D} \alpha(\theta, \phi)=c_{\mathcal{V}}(\theta ; \phi-\omega \theta)$, we have $\alpha(\theta, \phi)=\sqrt{2 J} \sin (\phi-\theta) /(\omega-1)$. Then (6.28) gives $\mathcal{U}(\theta, \phi)=\exp \{J(\sqrt{2 J} /(\omega-1)) \sin (\phi-\theta)\}$. One observes that $\mathcal{U}$ is a uniform IFF and $C_{\mathcal{U}}=0$. Hence, by Theorem 6.3(a) the torus is well-tuned and on a spin-orbit resonance.

(14) In Theorems 6.3-6.5, we assumed that an ISF exists and for the physically interesting $\mathcal{A}$ 's we hope this is true. It is also clear from Sec. III that for $J=0$ an ISF does exist. Nevertheless, for $J \neq 0$ this assumption in the theorems is not superfluous as we will now show by constructing, for a fixed torus, an $\mathcal{A}$ such that proper UPFs exist at every $\phi_{0}$ but an ISF does not.

We consider the case where $d=1, \omega=1$ and where $\mathcal{A}(\theta, \phi)$ depends only on $\phi-\theta$ and is given by

$$
\begin{aligned}
\mathcal{A}(\theta, \phi) & \equiv \hat{\mathcal{A}}(\phi-\theta) \\
& :=\left(\begin{array}{ccc}
0 & -\Omega_{3}(\phi-\theta) & \Omega_{2}(\phi-\theta) \\
\Omega_{3}(\phi-\theta) & 0 & -\Omega_{1}(\phi-\theta) \\
-\Omega_{2}(\phi-\theta) & \Omega_{1}(\phi-\theta) & 0
\end{array}\right) .
\end{aligned}
$$

Here the function $\Omega$ is smooth and $2 \pi$-periodic and we assume $|\Omega|<1$. The principal solution matrix is given by $\Phi\left(\theta ; \phi_{0}\right)=\exp \left(\hat{\mathcal{A}}\left(\phi_{0}\right) \theta\right)$. Because $\hat{\mathcal{A}}$ is skew-symmetric with spectrum $\lambda\left(\hat{\mathcal{A}}\left(\phi_{0}\right)\right)=$ $\left\{i\left|\Omega\left(\phi_{0}\right)\right|,-i\left|\Omega\left(\phi_{0}\right)\right|, 0\right\}$, it follows by Lemma 2.1(b) that a $\mathrm{SO}(3)$ matrix $W\left(\phi_{0}\right)$ exists such that

$$
\hat{\mathcal{A}}\left(\phi_{0}\right)=W\left(\phi_{0}\right)\left|\Omega\left(\phi_{0}\right)\right| J W^{T}\left(\phi_{0}\right) .
$$

Hence,

$$
\Phi\left(\theta ; \phi_{0}\right)=W\left(\phi_{0}\right) \exp \left(J\left|\Omega\left(\phi_{0}\right)\right| \theta\right) W^{T}\left(\phi_{0}\right),
$$

so that $W\left(\phi_{0}\right)$ is a proper UPF at $\phi_{0}$ with UPR $\left|\Omega\left(\phi_{0}\right)\right|$.

We now proceed to construct an $\Omega$ such that an ISF does not exist. If an ISF $n$ exists, then, by (6.3) and Theorem 6.1, we have

$$
\Phi\left(2 \pi ; \phi_{0}\right) n\left(0, \phi_{0}\right)=n\left(0, \phi_{0}+2 \pi \omega\right),
$$

for all $\phi_{0}$. Then, by (6.35),

$$
\exp \left(J\left|\Omega\left(\phi_{0}\right)\right| 2 \pi\right) W^{T}\left(\phi_{0}\right) n\left(0 ; \phi_{0}\right)=W^{T}\left(\phi_{0}\right) n\left(0 ; \phi_{0}\right) .
$$

If $\left|\Omega\left(\phi_{0}\right)\right| \neq$ integer, i.e., if $\Omega\left(\phi_{0}\right) \neq 0$, then every eigenvector of $\exp \left(J\left|\Omega\left(\phi_{0}\right)\right| 2 \pi\right)$ for eigenvalue 1 is parallel to $(0,0,1)$. In addition, since $\hat{\mathcal{A}}\left(\phi_{0}\right) \Omega\left(\phi_{0}\right)=0,(6.34)$ implies that

$$
J W^{T}\left(\phi_{0}\right) \Omega\left(\phi_{0}\right)=0 .
$$

It follows that $W^{T}\left(\phi_{0}\right) n\left(0 ; \phi_{0}\right)$ and $W^{T}\left(\phi_{0}\right) \Omega\left(\phi_{0}\right)$ are parallel to $(0,0,1)$. Hence $n\left(0 ; \phi_{0}\right)=$ const $\times \Omega\left(\phi_{0}\right)$. We conclude that for $\Omega\left(\phi_{0}\right) \neq 0$,

$$
n\left(0 ; \phi_{0}\right)= \pm \Omega\left(\phi_{0}\right) /\left|\Omega\left(\phi_{0}\right)\right| .
$$

Thus if an ISF $n$ exists, then 


$$
n\left(0, \phi_{0}\right)= \begin{cases} \pm \Omega\left(\phi_{0}\right) /\left|\Omega\left(\phi_{0}\right)\right| & \text { if } \Omega\left(\phi_{0}\right) \neq 0 \\ \xi & \text { if } \Omega\left(\phi_{0}\right)=0,\end{cases}
$$

where $\xi$ is arbitrary of norm one. To obtain our example, we present a smooth $\Omega$ such that $\Omega\left(\phi_{0}\right) /\left|\Omega\left(\phi_{0}\right)\right|$ does not have a continuous extension to those $\phi_{0}$, where $\Omega\left(\phi_{0}\right)=$ 0 . Let $\psi(x):=1 / \sin (x)$ and

$$
\Omega\left(\phi_{0}\right):= \begin{cases}(1 / 2) \sin ^{3}\left(\phi_{0}\right)\left[\cos \left(\psi\left(\phi_{0}\right)\right),-\sin \left(\psi\left(\phi_{0}\right)\right), 0\right] & \text { if } \sin \left(\phi_{0}\right) \neq 0 \\ 0 & \text { if } \sin \left(\phi_{0}\right)=0 .\end{cases}
$$

It is easy to show that $\Omega$ is smooth with $|\Omega|<1$ so that $\mathcal{A}(\theta, \phi)$ is smooth and $2 \pi$-periodic. However,

$$
\Omega\left(\phi_{0}\right) /\left|\Omega\left(\phi_{0}\right)\right|=\frac{\sin \left(\phi_{0}\right)}{\left|\sin \left(\phi_{0}\right)\right|}\left[\cos \left(\psi\left(\phi_{0}\right)\right),-\sin \left(\psi\left(\phi_{0}\right)\right), 0\right], \quad \sin \left(\phi_{0}\right) \neq 0
$$

does not have a continuous extension to all of $\phi_{0}$. Thus there is no ISF.

In summary, we have an example of a torus on orbital resonance for which a proper UPF exists at each $\phi_{0}$ but which is ill-tuned because the set $\left\{(1 / 2)\left|\sin ^{3}\left(\phi_{0}\right)\right|: \phi_{0} \in\right.$ $\mathbb{R}\}$ is a subset of $\Xi$ and has uncountably many elements. Furthermore, there is no ISF and thus no IFF. Of course, an $\mathcal{A}$ as exotic as that defined here will not emerge from the fields of a real storage ring.

(15) Now that IFFs are available we present another generalization of the Floquet theorem. Starting with (6.29) and assuming that the conditions of Theorem 6.5(a) hold, we write

$$
\begin{aligned}
\Phi\left(\theta ; \phi_{0}\right)= & U\left(\theta ; \phi_{0}\right) \exp \left(J \nu_{\mathcal{V}}\left(\phi_{0}\right) \theta\right) U^{T}\left(0 ; \phi_{0}\right) \\
= & U\left(\theta ; \phi_{0}\right) U^{T}\left(0 ; \phi_{0}\right) U\left(0 ; \phi_{0}\right) \\
& \times \exp \left(J \nu_{\mathcal{V}}\left(\phi_{0}\right) \theta\right) U^{T}\left(0 ; \phi_{0}\right) \\
= & p\left(\theta ; \phi_{0}\right) \exp \left(B\left(\phi_{0}\right) \nu\left(\phi_{0}\right) \theta\right),
\end{aligned}
$$

where

$$
\begin{aligned}
& p\left(\theta ; \phi_{0}\right):=U\left(\theta ; \phi_{0}\right) U^{T}\left(0 ; \phi_{0}\right), \\
& B\left(\phi_{0}\right):=U\left(0 ; \phi_{0}\right) J U^{T}\left(0 ; \phi_{0}\right), \quad \nu\left(\phi_{0}\right):=\nu_{\mathcal{V}}\left(\phi_{0}\right),
\end{aligned}
$$

and where $U$ is given by (6.30). Thus $U$ is a proper UPF. It follows from Remark 8 of Sec. V that $p\left(\cdot ; \phi_{0}\right), B\left(\phi_{0}\right), \nu\left(\phi_{0}\right)$ are generalized Floquet parameters at $\phi_{0}$. Thus under the conditions of Theorem 6.5(a) we have generalized Floquet parameters and have obtained a generalization of the Floquet theorem at every $\phi_{0}$. Note that $\nu_{\mathcal{V}}\left(\phi_{0}\right)$ is given by (6.26).

(16) For speed and practicality, simulations of particle motion in storage rings are often made with the approximations that the fields at the ends of magnets fall to zero abruptly (the "hard edge approximation") or that a magnet has zero length but the correct field integral (the "thin lens approximation"). Then the $\mathcal{A}$ 's are not smooth in $\theta$ so that the theory of this paper would have to be modified. For example, an ISF would be defined without imposing smoothness in $\theta$. The IFF would also not be smooth in $\theta$. We return to these matters in Sec. X.

We now prove a simple partial converse of Theorem 6.3(a), which gives a large class of well-tuned $\mathcal{A}$ 's with a uniform IFF.
Theorem 6.7 Let $q(\theta, \phi)$ be a $\mathrm{SO}(3)$ matrix which is of class $C^{2}$ and $2 \pi$-periodic such that $q(0, \phi)=I$, and let $b$ be a constant real skew-symmetric matrix. Let $A$ be defined by (5.2) with $\mathcal{A}=(\mathcal{D} q+q b) q^{T}$. Then a uniform IFF exists, so that the torus is well-tuned.

Proof: Because $b$ is skew-symmetric, by Lemma 2.1(b) a constant $\mathrm{SO}(3)$ matrix $W$ exists such that $b=$ $(\nu+m) W J W^{T}$, where $\nu \in[0,1)$ and the integer $m$ is constant. Defining $\mathcal{V}:=q W \exp (\operatorname{Jm} \theta)$, we observe that the smooth $\operatorname{SO}(3)$ function $\mathcal{V}(\theta, \phi)$ is $2 \pi$-periodic and that $\mathcal{D} \mathcal{V}=\mathcal{A} \mathcal{V}-\nu \mathcal{V} J$. Hence $C_{\mathcal{V}}=\nu$. For the third column of $\mathcal{V}$ we have

$$
\mathcal{D} \mathfrak{v}^{3}=\mathcal{D} \mathcal{V}(0,0,1)=\mathcal{A} \mathcal{V}(0,0,1) .
$$

So it is an ISF and therefore $\mathcal{V}$ is an IFF. The IFF $\mathcal{V}$ is uniform so that by Theorem 6.3(a) the torus is welltuned.

\section{Remark}

(18) We obtain the principal solution matrix as in (6.29) with $\nu_{\mathcal{V}}=\nu$ and

$$
U\left(\theta ; \phi_{0}\right)=q\left(\theta, \omega \theta+\phi_{0}\right) W \exp (J m \theta) .
$$

Combining (6.29) and (6.38) gives the generalized Floquet form

$$
\begin{aligned}
\Phi\left(\theta ; \phi_{0}\right) & =q\left(\theta, \omega \theta+\phi_{0}\right) W \exp (J(m+\nu) \theta) W^{T} \\
& =q\left(\theta, \omega \theta+\phi_{0}\right) \exp (b \theta) .
\end{aligned}
$$

We now apply some ideas of this section to some more simple models. Then in Sec. IX we return to the general case and consider the spectrum of the principal solution matrix.

\section{THE SINGLE RESONANCE MODEL}

In this section we examine the so-called "single resonance model." This model provides an approximation for the matrix $\mathcal{A}$ and it is frequently used in approximate descriptions of spin-orbit resonance effects both in proton and electron rings $[6,49]$. The reason for its popularity is that it can be solved exactly and delivers useful indications about behavior near spin-orbit resonance even 
though $\mathcal{A}$ is an approximation. Since it can be solved exactly it provides an example for $\Phi\left(\theta ; \phi_{0}\right)$ in (6.29) and (6.30).

The single resonance model effectively describes the spin motion for a particle limited to a harmonic vertical betatron oscillation around a horizontal circular design orbit. Thus there is only one pair of action-angle variables $J, \phi$, and $d=1$. The matrix $\mathcal{A}$ then contains terms due to a constant vertical magnetic field and terms due to a radial quadrupole field oscillating with the tune $\omega=$ $d \phi / d \theta$. The radial field can be decomposed into two counterrotating horizontal components rotating with the tune $\omega$. Close to resonance it is sufficient to consider just one of the rotating field components and to neglect the other $[15,49]$. The matrix $\mathcal{A}$ then takes the form

$$
\begin{aligned}
& \star \star \star \mathcal{A}(\phi):= \\
& \left(\begin{array}{ccc}
0 & -\sigma_{1} & \sigma_{2} \sqrt{2 J} \sin (\phi) \\
\sigma_{1} & 0 & -\sigma_{2} \sqrt{2 J} \cos (\phi) \\
-\sigma_{2} \sqrt{2} \operatorname{J} \sin (\phi) \sigma_{2} \sqrt{2 J} \cos (\phi) & 0
\end{array}\right),
\end{aligned}
$$

where $\sigma_{1}$ and $\sigma_{2}$ are real constants describing the strength of the vertical field and of the rotating horizontal field, respectively. Note that $\mathcal{A}$ is a smooth function of $\phi$, independent of $\theta$.

Our first aim is to show that a proper UPF exists at every $\phi_{0}$. We first consider the case $\sigma_{1} \neq \omega, \sigma_{2} \sqrt{2 J} \neq 0$ and define $\sigma:=\sqrt{\left(\sigma_{1}-\omega\right)^{2}+2 \sigma_{2}^{2} J}$. Then it is easy to verify that (see also [6])

$$
\mathfrak{v}^{3}(\phi)=\frac{1}{\sigma}\left(\begin{array}{c}
\sigma_{2} \sqrt{2 J} \cos (\phi) \\
\sigma_{2} \sqrt{2 J} \sin (\phi) \\
\sigma_{1}-\omega
\end{array}\right) .
$$

This is $2 \pi$-periodic and it satisfies (6.1). It is thus an ISF. Since $(1,0,0) \times \mathfrak{v}^{3}(\phi) \neq 0$, we can define $\mathfrak{v}^{2}(\phi) \perp \mathfrak{v}^{3}(\phi)$ by

$$
\begin{aligned}
\mathfrak{v}^{2}(\phi) & :=\frac{(1,0,0) \times \mathfrak{v}^{3}(\phi)}{\left|(1,0,0) \times \mathfrak{v}^{3}(\phi)\right|} \\
& =\frac{1}{\sqrt{\sigma^{2}-2 \sigma_{2}^{2} J \cos ^{2}(\phi)}}\left(\begin{array}{c}
0 \\
\omega-\sigma_{1} \\
\sigma_{2} \sqrt{2 J} \sin (\phi)
\end{array}\right),
\end{aligned}
$$

and $\mathfrak{v}^{1}(\phi)$ by $\mathfrak{v}^{1}:=\mathfrak{v}^{2} \times \mathfrak{v}^{3}$. Thus $\mathcal{V}:=\left[\mathfrak{v}^{1}, \mathfrak{v}^{2}, \mathfrak{v}^{3}\right]$ is smooth and $2 \pi$-periodic. It is thus an IFF. By (6.15), (7.2), and (7.3) we obtain

$$
\begin{aligned}
C_{\mathcal{V}}(\phi) & =\Omega(\phi) \cdot \mathfrak{v}^{3}(\phi)+\mathfrak{v}^{1}(\phi) \cdot\left(\mathcal{D v}^{2}(\phi)\right) \\
& =\sigma+\frac{\sigma \omega\left(\sigma_{1}-\omega\right)}{\sigma^{2}-2 \sigma_{2}^{2} J \cos ^{2}(\phi)}
\end{aligned}
$$

so that

$$
\begin{aligned}
\bar{c}_{\mathcal{V}}\left(\phi_{0}\right) & =\lim _{T \rightarrow \infty}(1 / T) \int_{0}^{T} d \theta^{\prime} C_{\mathcal{V}}\left(\omega \theta^{\prime}+\phi_{0}\right) \\
& =(1 / 2 \pi) \int_{0}^{2 \pi} d \phi C_{\mathcal{V}}(\phi),
\end{aligned}
$$

where we use the $2 \pi$-periodicity of $C_{\mathcal{V}}$. Thus $\bar{c} \mathcal{V}\left(\phi_{0}\right)$ is independent of $\phi_{0}$. Since ([50], p. 379)

$$
\int_{0}^{\pi / 2} \frac{d \phi}{\left(\sigma_{1}-\omega\right)^{2} \sin ^{2}(\phi)+\sigma^{2} \cos ^{2}(\phi)}=\frac{\pi}{2 \sigma\left|\sigma_{1}-\omega\right|},
$$

and since by (7.4) and (7.5)

$$
\begin{aligned}
\bar{c}_{\mathcal{V}}= & \sigma+\frac{\sigma \omega\left(\sigma_{1}-\omega\right)}{2 \pi} \\
& \times \int_{0}^{2 \pi} \frac{d \phi}{\left(\sigma_{1}-\omega\right)^{2} \cos ^{2}(\phi)+\sigma^{2} \sin ^{2}(\phi)} \\
= & \sigma+\frac{\sigma \omega\left(\sigma_{1}-\omega\right)}{\pi} \int_{0}^{\pi} \frac{d \phi}{\left(\sigma_{1}-\omega\right)^{2} \cos ^{2}(\phi)+\sigma^{2} \sin ^{2}(\phi)} \\
= & \sigma+\frac{2 \sigma \omega\left(\sigma_{1}-\omega\right)}{\pi} \\
& \times \int_{0}^{\pi / 2} \frac{d \phi}{\left(\sigma_{1}-\omega\right)^{2} \sin ^{2}(\phi)+\sigma^{2} \cos ^{2}(\phi)}
\end{aligned}
$$

we can make the assignments

$$
\begin{aligned}
\bar{c}_{\mathcal{V}} & =\sigma+\omega \operatorname{sgn}\left(\sigma_{1}-\omega\right), \\
\tilde{c}_{\mathcal{V}}(\phi) & =\frac{\sigma \omega\left(\sigma_{1}-\omega\right)}{\sigma^{2}-2 \sigma_{2}^{2} J \cos ^{2}(\phi)}-\omega \operatorname{sgn}\left(\sigma_{1}-\omega\right) .
\end{aligned}
$$

Because $\tilde{c} \mathcal{V}$ does not depend on $\theta$ one finds that (6.24) has smooth solutions $\alpha(\phi)$ which do not depend on $\theta$ and that one of those solutions reads as

$$
\alpha(\phi)=\frac{1}{\omega} \int_{0}^{\phi} d \phi^{\prime} \tilde{c} \mathcal{V}\left(\phi^{\prime}\right) .
$$

Since $0=\int_{0}^{2 \pi} d \phi \tilde{c} \mathcal{V}(\phi)$ and by the $2 \pi$-periodicity of $\tilde{c} \mathcal{V}(\phi)$ it follows by (7.8) that $\alpha(\phi)$ is $2 \pi$-periodic in $\phi$. Thus Theorem 6.5(a) applies and one concludes that $U\left(\cdot ; \phi_{0}\right)$, defined by

$$
\begin{aligned}
& U\left(\theta ; \phi_{0}\right) \\
& =\mathcal{V}\left(\phi_{0}+\omega \theta\right) \exp \left\{J\left(\frac{1}{\omega} \int_{\phi_{0}}^{\phi_{0}+\omega \theta} d \phi^{\prime} \tilde{c}_{\mathcal{V}}\left(\phi^{\prime}\right)+k_{\mathcal{V}} \theta\right)\right\}
\end{aligned}
$$

is a proper UPF at $\phi_{0}$ with UPR $\nu_{\mathcal{V}}$. Here $\nu_{\mathcal{V}}$ and the integer $k_{\mathcal{V}}$ are uniquely determined by $\bar{c}_{\mathcal{V}}$ via (6.26) and (7.7). Because $\nu_{\mathcal{V}}$ is contained in each $\Xi\left(\phi_{0}\right)$ we conclude (recall the comment after Definition 5.4) that the tori are well-tuned when $\sigma_{1} \neq \omega$ and $\sigma_{2} \sqrt{2 J} \neq 0$.

We now discuss the general case using the machinery of Sec. VI.

Proposition 7.1 The single resonance model has a uniform IFF for every value $J$ of the orbital action variable. Hence the corresponding torus is well-tuned. 
Proof: For $\mathcal{Y}(\theta, \phi):=\exp (\phi J)$ one obtains

$$
\mathcal{Y}^{T}(\mathcal{A} \mathcal{Y}-\mathcal{D} \mathcal{Y})=E,
$$

where

$$
E:=\left(\begin{array}{ccc}
0 & -\sigma_{1}+\omega & 0 \\
\sigma_{1}-\omega & 0 & -\sigma_{2} \sqrt{2 J} \\
0 & \sigma_{2} \sqrt{2 J} & 0
\end{array}\right) .
$$

This has eigenvalues $\lambda(E)=\{i \sigma,-i \sigma, 0\}$. If $\sigma=0$, then $E=0$ and, due to (6.6) and (7.10), $\mathcal{Y}$ is a uniform IFF and $C_{y}=0$. Thus by Theorem 6.3(a) the tori are well-tuned and 0 is a spin tune. If $\sigma \neq 0$, then by Lemma 2.1(b) we can choose $W \in \mathrm{SO}(3)$ such that

$$
W^{-1} E W=\sigma J=:(\nu+k) J,
$$

where the integer $k$ is chosen such that $\nu \in[0,1)$. We now define

$$
\mathcal{U}(\theta ; \phi):=\mathcal{Y}(\theta ; \phi) W \exp (k \theta J),
$$

and obtain

$$
\mathcal{U}^{T}(\mathcal{A} U-\mathcal{D} \mathcal{U})=\nu J
$$

It follows by (6.6) that $\mathcal{U}$ is a uniform IFF and $C_{U}=$ $\nu=\sigma-k$. Thus by Theorem 6.3(a) the tori are welltuned and $\sigma-k$ is a spin tune.

\section{Remarks}

(1) The proof of Proposition 7.1 and Definition 5.5 show that the spin tunes $\omega_{s}$ of the single resonance model have the form

$$
\omega_{s}=\varepsilon \sigma+j \omega+k,
$$

where $\varepsilon \in\{-1,1\} ; j, k \in \mathbb{Z}$. Conversely, every constant of the form (7.11) is a spin tune of the single resonance model, if it is in $[0,1)$. In particular, the set $\Xi\left(\phi_{0}\right)$ is independent of $\phi_{0}$. Note that for the single resonance model, spin tunes exist also on orbital resonance, i.e., for rational $\omega$; see also Remark 4.

(2) The case $\sigma_{2} \sqrt{2 J}=0$ represents the absence of betatron motion, i.e., motion on the design orbit. In this case $\varepsilon, j, k$ can be chosen in (7.11) such that the spin tune reduces to $\sigma_{1}$.

(3) From the expression for $\sigma$ it is clear that, during variation of $\sigma_{1}$, the spin tune in (7.11) comes closest to the spin-orbit resonance $\omega_{s}=k+j \omega$ when $\sigma_{1}=\omega$. However, for the case $\sigma_{2} \sqrt{2 J} \neq 0$ of principle interest, the resonance condition is not reached.

(4) If $\omega$ is an integer, the matrix $A$ is one-turn periodic and the ISF can be obtained as the eigenvector of length 1 of the one-turn principal solution matrix. Moreover, every proper UPF is one-turn periodic and its UPR can be obtained from the complex eigenvalues of the one-turn principal solution matrix just as in Sec. III. Of course, this UPR is a spin tune. If $\omega$ is rational, the ISF can be obtained as the eigenvector of length 1 of the appropriate multiturn principal solution matrix; see, e.g., [51]. Every proper UPF is then multiturn periodic and its UPR is extracted from the corresponding complex eigenvalues and is again a spin tune. Note that this circumstance that a spin tune exists even on orbital resonance has its origin in the facts that the single resonance model has only one orbital frequency and that $\mathcal{A}$ is independent of $\theta$.

(5) If the orbital tune is rational, then with Proposition 7.1 we have an example of a torus which is on orbital resonance but is nevertheless well-tuned. This is an example for which the torus may be on orbital resonance but still satisfy the conditions of Theorem 6.3(a).

\section{THE MOSER-SIEGEL MODEL}

In this section we construct and study an illustrative but unphysical model which, for reasons which will become clear, we will call the "Moser-Siegel model." This model has $d=1$, can be solved exactly, and has two real parameters $\sigma_{1}, \sigma_{2}$, where $\sigma_{1}>1$. For certain choices of the orbital tune $\omega$ and of $\sigma_{1}, \sigma_{2}$, the MoserSiegel model provides an example of spin motion which is nonquasiperiodic. The matrix $\mathcal{A}$ of the Moser-Siegel model takes the form

$$
\begin{aligned}
& \star \star \star \quad \mathcal{A}:=\mathcal{A}_{21} J, \\
& \mathcal{A}_{21}(\theta, \phi):=\sum_{k, l=1}^{\infty} \sigma_{1}^{-k-l} \eta_{k l} \sin (k \phi-l \theta),
\end{aligned}
$$

where

$$
\eta_{k l}:= \begin{cases}1 & \text { if } k \sigma_{2}-l \geq 0 \\ -1 & \text { if } k \sigma_{2}-l<0,\end{cases}
$$

and by definition $\mathcal{A}$ is smooth (in fact $C^{\infty}$ ) and $2 \pi$-periodic. The latter follows from the convergence of $\sum_{k=1}^{\infty} k^{n} \sigma_{1}^{-k}$ for a non-negative integer $n$, which follows from the ratio test and which implies that the series in (8.1) and the series of all its derivatives converge uniformly (see [46], Sec. 8.6.3, and [47], p. 117).

For $\phi_{0}=0$,

$$
\begin{aligned}
& A(\theta ; 0)=f(\theta) J, \\
& f(\theta)=\sum_{k, l=1}^{\infty} \sigma_{1}^{-k-l} \eta_{k l} \sin ((k \omega-l) \theta) .
\end{aligned}
$$

Clearly $f \in \mathcal{Q}(1, \omega ; 2)$ and for an irrational $\omega, \bar{f}=0$ by Lemma 4.3(c), so that

$$
\Phi(\theta ; 0)=\exp (J g(\theta)),
$$

where

$$
g(\theta):=\int_{0}^{\theta} \tilde{f}\left(\theta^{\prime}\right) d \theta^{\prime} .
$$


Because the function $g$ has been used in [52], paragraph 36, we call our model the Moser-Siegel model.

Proposition 8.1 For some $\omega \notin \mathbb{Q}$ there exist values of $\sigma_{1}, \sigma_{2}$ such that $g$ is unbounded. For these values, $\exp (i g)$ is not quasiperiodic whence $\Phi(\cdot ; 0)$ is not quasiperiodic. Proof: It is shown in [52], paragraph 36, that values of $\sigma_{1}, \sigma_{2}$ and of $\omega \notin \mathbb{Q}$ exist such that $g$ is unbounded. Thus, for these values, $\tilde{f}$ is an almost periodic function whose integral, $g$, is unbounded. It then follows (see [44], Chap. 6) that $\exp (\mathrm{ig})$ is not almost periodic, whence at least one of $\cos (g)$ and $\sin (g)$ is not almost periodic.

Remarks

(1) Proposition 8.1 shows that for certain values of $\sigma_{1}, \sigma_{2}$ and of $\omega$ the principal solution matrix at $\phi_{0}=0$ is not quasiperiodic, so that $\Xi\left(\phi_{0}=0\right)=\emptyset$. In particular, for those values the torus is ill-tuned so that, by Theorem 6.3(a), it has no uniform IFF.

(2) The unit matrix obviously provides an IFF for the Moser-Siegel model. Thus Proposition 8.1 demonstrates that the existence of an IFF is neither sufficient for having a uniform IFF nor for having a well-tuned torus. In the context of Theorem 6.5(a) this means that the existence of an IFF does not necessarily admit a smooth solution $\alpha$ of (6.24), which is $2 \pi$-periodic.

\section{SPECTRAL ANALYSIS OF QUASIPERIODIC SPIN MOTION}

In the code SPRINT, ISFs are calculated nonperturbatively in three ways, namely, by stroboscopic averaging, by the SODOM2 algorithm, and by adiabatic antidamping $[6,8,9,12,13,23,25]$. Perturbative algorithms for obtaining ISFs are listed in the Introduction. Moreover, the spin tune is calculated nonperturbatively in SPRINT by logging the spin precession around the ISF or by the SODOM2 algorithm. We will make further comments on these simulations in Sec. X.

The fact that under appropriate conditions the set of generalized Floquet frequencies is the set of spin tunes suggests a further way to obtain the spin tune, namely, by spectral analysis of quasiperiodic functions. The way forward is contained in the following theorem. As we shall see, we can also use spectral analysis to construct the ISF.

Theorem 9.1 Consider a uniform IFF $\mathcal{V}$ on a fixed torus. Then the following holds.

(a) Let $S$ be a spin trajectory at $\phi_{0}$. If $0 \in\left[C_{V}\right]$, where the equivalence class $\left[C_{V}\right]$ is defined in $S e c . \mathrm{V}$, then $S$ is in $Q(1, \omega ; d+1)$.

(b) Let $S$ be a spin trajectory at $\phi_{0}$ and let the torus be off orbital resonance. Also, let $0 \notin\left[C_{\mathcal{V}}\right]$. Then $\hat{S}$, defined by

$\hat{S}(\theta):=\lim _{N \rightarrow \infty} \sum_{\substack{m \in \mathbb{Z}^{+}+1 \\\|m\| \leq N}} A_{N, m} \exp (i \theta m \cdot(1, \omega)) a(S, m \cdot(1, \omega))$, with $A_{N, m}:=\prod_{n=1}^{d+1} \frac{N+1-\left|m_{n}\right|}{N+1}$, satisfies the relation

$$
\hat{S}(\theta)=n\left(\theta, \phi_{0}+\omega \theta\right) \hat{S}(0) \cdot n\left(0, \phi_{0}\right),
$$

where $n$ is the ISF $n:=\mathcal{V}(0,0,1)$. Moreover, $\hat{S}$ is a spin trajectory in $\mathcal{Q}(1, \omega ; d+1)$.

(c) For arbitrary $\phi_{0}$,

$\star \star \star \quad \Lambda\left(\Phi\left(\cdot ; \phi_{0}\right)\right)$

$$
\subset\left\{\varepsilon C_{\mathcal{V}}+m \cdot(1, \omega):(\varepsilon, m) \in\{0,1,-1\} \times \mathbb{Z}^{d+1}\right\} .
$$

Proof of Theorem 9.1(a): For fixed $\phi_{0}$, define $U(\theta):=$ $\mathcal{V}\left(\theta, \phi_{0}+\omega \theta\right)$ so that by Theorem 6.3(a) $U$ is a proper $\mathrm{UPF}$ at $\phi_{0}$ and we have

$$
\Phi\left(\theta ; \phi_{0}\right)=U(\theta) \exp \left(J C_{\mathcal{V}} \theta\right) U^{T}(0) .
$$

Thus if $0 \in\left[C_{\mathcal{V}}\right]$, then $\Phi\left(\cdot ; \phi_{0}\right)$, and therefore $S$, is in $Q(1, \omega ; d+1)$.

Proof of Theorem 9.1(b): Because the $U$, defined in the proof of Theorem 9.1(a), is in $\mathcal{Q}(1, \omega ; d+1)$ and because the torus is off orbital resonance we can use Lemmas 4.3(b) and 4.3(c) to write

$$
U(\theta)=\lim _{N \rightarrow \infty} \sum_{\substack{m \in Z^{d+1} \\\|m\| \leq N}} A_{N, m} \exp (i \theta m \cdot(1, \omega)) a(U, m \cdot(1, \omega)) .
$$

With

$$
\Delta_{ \pm}:=\frac{1}{2}\left(\begin{array}{ccc}
1 & \pm i & 0 \\
\mp i & 1 & 0 \\
0 & 0 & 0
\end{array}\right), \quad \Delta_{0}:=\left(\begin{array}{ccc}
0 & 0 & 0 \\
0 & 0 & 0 \\
0 & 0 & 1
\end{array}\right)
$$

and recalling (2.12), we have

$\exp \left(J C_{\mathcal{V}} \theta\right)=\Delta_{+} \exp \left(i C_{\mathcal{V}} \theta\right)+\Delta_{-} \exp \left(-i C_{\mathcal{V}} \theta\right)+\Delta_{0}$

so that

$$
\begin{aligned}
a\left(U \exp \left(J C_{\mathcal{V}} \cdot\right), m \cdot(1, \omega)\right)= & a\left(U \Delta_{+}, m \cdot(1, \omega)-C_{\mathcal{V}}\right) \\
& +a\left(U \Delta_{-}, m \cdot(1, \omega)+C_{\mathcal{V}}\right) \\
& +a\left(U \Delta_{0}, m \cdot(1, \omega)\right)
\end{aligned}
$$

Since $0 \notin\left[C_{\mathcal{V}}\right]$, then $\pm C_{\mathcal{V}} \notin\left\{m \cdot(1, \omega): m \in \mathbb{Z}^{d+1}\right\}$. It follows from Lemma 4.3(e) that $\pm C_{\mathcal{V}} \notin \Lambda(U)$. Then $0=$ $a\left(U, m \cdot(1, \omega) \mp C_{\mathcal{V}}\right)$ so that (9.7) leads to

$$
a\left(U \exp \left(J C_{\mathcal{V}}\right), m \cdot(1, \omega)\right)=a\left(U \Delta_{0}, m \cdot(1, \omega)\right) .
$$

Combining (9.3), (9.4), and (9.8) we obtain 


$$
\begin{aligned}
U(\theta) \Delta_{0}= & \lim _{N \rightarrow \infty} \sum_{\substack{m \in \in^{d}+1 \\
\|m\| \leq N}} A_{N, m} \exp (i \theta m \cdot(1, \omega)) a\left(U \Delta_{0}, m \cdot(1, \omega)\right) \\
= & \lim _{N \rightarrow \infty} \sum_{\substack{m \in \mathbb{Z}^{d}+1 \\
\|m\| \leq N}} A_{N, m} \exp (i \theta m \cdot(1, \omega)) \\
& \times a\left(U \exp \left(J C_{V} \cdot\right), m \cdot(1, \omega)\right) \\
= & \lim _{N \rightarrow \infty} \sum_{\substack{m \in \mathbb{Z}^{d}+1 \\
\|m\| \leq N}} A_{N, m} \exp (i \theta m \cdot(1, \omega)) \\
& \times a\left(\Phi\left(\cdot ; \phi_{0}\right) U(0), m \cdot(1, \omega)\right)
\end{aligned}
$$

Hence,

$$
\begin{aligned}
& U(\theta) \Delta_{0} U^{T}(0) \\
& \quad=\lim _{N \rightarrow \infty} \sum_{\substack{m \in \mathbb{Z}^{d+1} \\
\|m\| \leq N}} A_{N, m} \exp (i \theta m \cdot(1, \omega)) a\left(\Phi\left(\cdot ; \phi_{0}\right), m \cdot(1, \omega)\right) .
\end{aligned}
$$

Then, because $S$ is a spin trajectory at $\phi_{0}$,

$$
\begin{aligned}
U(\theta) \Delta_{0} U^{T}(0) S(0)= & \lim _{N \rightarrow \infty} \sum_{\substack{m \in \mathbb{Z}^{d+1} \\
\|m\| \leq N}} A_{N, m} \exp (i \theta m \cdot(1, \omega)) \\
& \times a\left(\Phi\left(\cdot ; \phi_{0}\right) S(0), m \cdot(1, \omega)\right) \\
= & \lim _{N \rightarrow \infty} \sum_{\substack{m \in \mathbb{Z}^{d+1} \\
\|m\| \leq N}} A_{N, m} \exp (i \theta m \cdot(1, \omega)) \\
& \times a(S, m \cdot(1, \omega))=\hat{S}(\theta),
\end{aligned}
$$

where the last equality follows from (9.1). From (2.17), (9.3), (9.5), and (9.10) it follows that

$$
\begin{aligned}
\dot{\hat{S}}(\theta) & =\dot{U}(\theta) \Delta_{0} U^{T}(0) S(0) \\
& =\left(A\left(\theta ; \phi_{0}\right) U(\theta)-C_{\mathcal{V}} U(\theta) J\right) \Delta_{0} U^{T}(0) S(0) \\
& =A\left(\theta ; \phi_{0}\right) U(\theta) \Delta_{0} U^{T}(0) S(0)=A\left(\theta ; \phi_{0}\right) \hat{S}(\theta) .
\end{aligned}
$$

Thus $\hat{S}$ is a spin trajectory at $\phi_{0}$. By (9.10) and because $U$ is proper, $\hat{S}$ is in $Q(1, \omega ; d+1)$.

If $S$ is in $Q(1, \omega ; d+1)$, then by Lemmas $4.3(\mathrm{~b})$ and 4.3(c)

$$
S(\theta)=\lim _{N \rightarrow \infty} \sum_{\substack{m \in \mathbb{Z}^{d+1} \\\|m\| \leq N}} A_{N, m} \exp (i \theta m \cdot(1, \omega)) a(S, m \cdot(1, \omega)),
$$

so that in this special situation, $\hat{S}=S$. Thus, for an arbitrary spin trajectory $S$ at $\phi_{0}$, the double transform $\hat{\hat{S}}$ of $S$ from the double application of (9.1) is equal to the single transform $\hat{S}$ so that (9.10) yields

$$
U(\theta) \Delta_{0} U^{T}(0) \hat{S}(0)=\hat{\hat{S}}(\theta)=\hat{S}(\theta)
$$

If $\theta=0$, Eq. (9.13) becomes an eigenproblem for $\hat{S}(0)$. The solution is $\hat{S}(0)= \pm|\hat{S}(0)| U(0)(0,0,1)$. Inserting this into (9.13) yields

$$
\begin{aligned}
\hat{S}(\theta) & = \pm|\hat{S}(0)| n\left(\theta, \phi_{0}+\omega \theta\right) \\
& =n\left(\theta, \phi_{0}+\omega \theta\right) \hat{S}(0) \cdot n\left(0, \phi_{0}\right),
\end{aligned}
$$

where $n$ denotes the third column of $\mathcal{V}$ and where $S$ is an arbitrary spin trajectory at $\phi_{0}$.

Proof of Theorem 9.1(c): By (9.3) and (9.6) we have

$$
\begin{aligned}
& \Phi\left(\theta ; \phi_{0}\right) \\
& \quad=U(\theta)\left(\Delta_{+} \exp \left(i C_{\mathcal{V}} \theta\right)+\Delta_{-} \exp \left(-i C_{\mathcal{V}} \theta\right)+\Delta_{0}\right) U^{T}(0) .
\end{aligned}
$$

If $\lambda \in \Lambda\left(U \Delta_{+} \exp \left(i C_{\mathcal{V}^{*}}\right) U^{T}(0)\right)$, then

$$
\lambda-C_{\mathcal{V}} \in \Lambda\left(U \Delta_{+} U^{T}(0)\right) \text {. }
$$

Because $U$ is proper and with Lemma 4.3(e), $\Lambda\left(U \Delta_{+} U^{T}(0)\right) \subset\left\{m \cdot(1, \omega): m \in \mathbb{Z}^{d+1}\right\}$. Thus with (9.16), $\lambda \in\left\{C_{\mathcal{V}}+m \cdot(1, \omega): m \in \mathbb{Z}^{d+1}\right\}$. Then

$\Lambda\left(U \Delta_{+} \exp \left(i C_{\mathcal{V}}\right) U^{T}(0)\right) \subset\left\{C_{\mathcal{V}}+m \cdot(1, \omega): m \in \mathbb{Z}^{d+1}\right\}$

In an analogous way,

$$
\begin{aligned}
& \Lambda\left(U \Delta_{-} \exp \left(-i C_{\mathcal{V}} \cdot\right) U^{T}(0)\right) \\
& \quad \subset\left\{-C_{\mathcal{V}}+m \cdot(1, \omega): m \in \mathbb{Z}^{d+1}\right\} .
\end{aligned}
$$

Moreover,

$$
\Lambda\left(U \Delta_{0} U^{T}(0)\right) \subset\left\{m \cdot(1, \omega): m \in \mathbb{Z}^{d+1}\right\} .
$$

Combining (9.15), (9.17), (9.18), and (9.19) gives

$$
\begin{aligned}
\Lambda\left(\Phi\left(\cdot ; \phi_{0}\right)\right) \subset & \left(\Lambda\left(U \Delta_{+} \exp \left(i C_{\mathcal{V}} \cdot\right) U^{T}(0)\right)\right. \\
& \cup \Lambda\left(U \Delta_{-} \exp \left(-i C_{\mathcal{V}} \cdot\right) U^{T}(0)\right) \\
& \left.\cup \Lambda\left(U \Delta_{0} U^{T}(0)\right)\right) \\
\subset & \left\{\varepsilon C_{\mathcal{V}}+m \cdot(1, \omega):(\varepsilon, m)\{0,1,-1\}\right. \\
& \left.\times \mathbb{Z}^{d+1}\right\} .
\end{aligned}
$$

\section{Remarks}

(1) Let the conditions of Theorem 9.1(b) hold and let the spin trajectory $S$ at $\phi_{0}$ be in $Q(1, \omega ; d+1)$. In this special situation (9.12) holds, i.e., (9.1) becomes the spectral expansion of $S$. However, in general $S$ is not in $\mathcal{Q}(1, \omega ; d+1)$, i.e., in general (9.1) is not the spectral expansion of $S$ because only the tunes $m \cdot(1, \omega)$ appear in (9.1).

If the conditions of Theorem 9.1(b) hold, then $\hat{S}$ is parallel to an ISF and with (9.1) and (9.2) one could, at least in principle, attempt to compute the ISF by doing numerical spectral analysis on an arbitrary spin trajectory. Of course, by Theorem 6.4, the ISF $n$ is unique up to a sign and, by Theorem 6.3(a), the torus is well-tuned. 
(2) Consider a fixed torus and assume that a uniform IFF exists so that, due to Theorem 6.3(a), the torus is welltuned. Then, due to Remark 8 in Sec. V, the set of generalized Floquet frequencies is the same at every $\phi_{0}$ and is identical with the set $\Xi$ of spin tunes. Therefore Theorem 9.1(c) implies, for arbitrary $\phi_{0}$, that for every $\lambda$ in $\Lambda\left(\Phi\left(\cdot ; \phi_{0}\right)\right) \backslash\left\{m \cdot(1, \omega): m \in \mathbb{Z}^{d+1}\right\}$ the fractional part of $\lambda$ is a generalized Floquet frequency at $\phi_{0}$, and in particular a spin tune. Thus as conjectured earlier, spin tunes can indeed be obtained by spectral analysis.

Theorem 9.1(c) addresses the spectrum $\Lambda\left(\Phi\left(\cdot ; \phi_{0}\right)\right)$ for the conditions stated but gives no information on its dependence on $\phi_{0}$. However, under certain conditions, the special parameter dependence of $A$ given in (5.4) guarantees that $\Lambda\left(\Phi\left(\cdot ; \phi_{0}\right)\right)$ is independent of $\phi_{0}$, as we show in the next theorem.

Consider a fixed torus and denote $\Phi(\theta+$ $\left.2 \pi N ; \phi_{0}\right) \Phi^{T}\left(2 \pi N ; \phi_{0}\right)$ by $\Pi\left(\theta ; \phi_{0}\right)$, where $N$ is an integer. We conclude from (5.1) and (5.4) that $\Pi$ satisfies the initial value problem

$$
\begin{aligned}
& \frac{\partial \Pi\left(\theta ; \phi_{0}\right)}{\partial \theta}=A\left(\theta ; 2 \pi N \omega+\phi_{0}\right) \Pi\left(\theta ; \phi_{0}\right), \\
& \Pi\left(0 ; \phi_{0}\right)=I .
\end{aligned}
$$

Because $\Phi\left(\theta ; 2 \pi N \omega+\phi_{0}\right)$ is the unique solution of this initial value problem (see also Sec. II), we obtain

$$
\Phi\left(\theta ; 2 \pi N \omega+\phi_{0}\right)=\Phi\left(\theta+2 \pi N ; \phi_{0}\right) \Phi^{T}\left(2 \pi N ; \phi_{0}\right),
$$

valid for arbitrary $\phi_{0}$ and arbitrary integer $N$. Thus the basic property of $A$ in (5.4) leads to the basic property of $\Phi$ as manifested in (9.21). It follows that if $\Phi(\cdot ; \phi)$ is known at a fixed $\phi_{0}$, then it is known for all $\phi=\phi_{0}+$ $2 \pi N \omega+2 \pi M$ with $M \in \mathbb{Z}^{d}$. Then if in addition $(1, \omega)$ is nonresonant it follows by continuity that it is known for all $\phi$ on the torus.

Theorem 9.2 Consider a fixed torus $J_{0}$ off orbital resonance and assume that a $\phi_{0}$ exists such that $\Xi\left(\phi_{0}\right) \neq \emptyset$. Then for every real $\lambda$ and all $\phi \in \mathbb{R}^{d}, a(\Phi(\cdot ; \phi), \lambda)$ exists and is continuous in $\phi$. Moreover, for all $\phi$, $\Lambda(\Phi(\cdot ; \phi))=\Lambda\left(\Phi\left(\cdot ; \phi_{0}\right)\right)$.

Proof: Since $\Phi\left(\cdot+2 \pi N ; \phi_{0}\right)$ is quasiperiodic, it is easy to see from Definition 4.1 that

$$
a\left(\Phi\left(\cdot+2 \pi N ; \phi_{0}\right), \lambda\right)=\exp (i \lambda 2 \pi N) a\left(\Phi\left(\cdot ; \phi_{0}\right), \lambda\right),
$$

and the basic identity (9.21) gives

$$
\begin{aligned}
& a\left(\Phi\left(\cdot ; \phi_{0}+2 \pi N \omega\right), \lambda\right) \\
& \quad=\exp (i \lambda 2 \pi N) a\left(\Phi\left(\cdot ; \phi_{0}\right), \lambda\right) \Phi^{T}\left(2 \pi N ; \phi_{0}\right) .
\end{aligned}
$$

Therefore,

$$
\Lambda\left(\Phi\left(\cdot ; \phi_{0}\right)\right)=\Lambda\left(\Phi\left(\cdot ; \phi_{0}+2 \pi N \omega+2 \pi M\right)\right),
$$

for all $N \in \mathbb{Z}, M \in \mathbb{Z}^{d}$, where we also used the fact that
$\Phi\left(\theta ; \phi_{0}\right)$ is $2 \pi$-periodic in $\phi_{0}$. Thus the spectrum of the principal solution matrix on the set

$$
D^{\prime}:=\left\{\phi_{0}+2 \pi N \omega+2 \pi M: N \in \mathbb{Z}, M \in \mathbb{Z}^{d}\right\}
$$

is the same as the spectrum of the principal solution matrix at $\phi_{0}$. Because $(1, \omega)$ is nonresonant, $D^{\prime}$ is dense in $\mathbb{R}^{d}$. Now fix $\lambda$ and let $h(\phi):=a(\Phi(\cdot ; \phi), \lambda)$ for $\phi \in$ $D^{\prime}$ and assume $h\left(\phi_{0}\right) \neq 0$ so that $\lambda \in \Lambda\left(\Phi\left(\cdot ; \phi_{0}\right)\right)$. Then

$$
\begin{aligned}
0<\left|h\left(\phi_{0}\right)\right| & =\left|h\left(\phi_{0}+2 \pi N \omega\right) \Phi\left(2 \pi N ; \phi_{0}\right)\right| \\
& \leq \sqrt{3}\left|h\left(\phi_{0}+2 \pi N \omega\right)\right| \\
& =\sqrt{3}\left|h\left(\phi_{0}+2 \pi N \omega+2 \pi M\right)\right|,
\end{aligned}
$$

where we used (9.23) for the first equality and where for the second inequality we used the fact that

$$
\left|X \Phi\left(2 \pi N ; \phi_{0}\right)\right| \leq \sqrt{3}|X|,
$$

which follows from the $\mathrm{SO}(3)$ nature of $\Phi$. As always, $|\cdot|$ denotes the Euclidean norm, i.e., $|X|:=$ $\sqrt{X_{11} X_{11}^{*}+X_{12} X_{12}^{*}+\cdots+X_{33} X_{33}^{*}}$. If $h$ is continuous and $2 \pi$-periodic and defined all over $\mathbb{R}^{d}$, then, since $D^{\prime}$ is dense in $\mathbb{R}^{d},|h(\phi)|>0$ for all $\phi$ and thus $\Lambda\left(\Phi\left(\cdot ; \phi_{0}\right)\right) \subset \Lambda(\Phi(\cdot ; \phi))$ for all $\phi$. Conversely, if, for a given $\phi,|h(\phi)|>0$, then, by exchanging the roles of $\phi$ and $\phi_{0}$, we obtain $\left|h\left(\phi_{0}\right)\right|>0$ so that $\Lambda\left(\Phi\left(\cdot ; \phi_{0}\right)\right) \supset$ $\Lambda(\Phi(\cdot ; \phi))$ for all $\phi$. Thus $\Lambda\left(\Phi\left(\cdot ; \phi_{0}\right)\right)=\Lambda(\Phi(\cdot ; \phi))$ for all $\phi$ and it remains to show that $h$ is defined and continuous on $\mathbb{R}^{d}$. The $2 \pi$-periodicity of $h$ then follows immediately. We first state the following lemma.

Lemma 9.3 For fixed $\lambda$ and nonresonant $(1, \omega)$, let

$$
\Phi_{n, \lambda}(\phi):=\frac{1}{n} \int_{0}^{n} \Phi(\theta ; \phi) \exp (-i \lambda \theta) d \theta
$$

converge uniformly for all $\phi$ in the set $D^{\prime}$ of (9.25) as the integer $n \rightarrow \infty$. Then $\Phi_{n, \lambda}$ converges uniformly on $\mathbb{R}^{d}$. In particular, a $(\Phi(\cdot ; \phi), \lambda)$ exists for all $\phi$ in $\mathbb{R}^{d}$ and is continuous in $\phi$.

Proof of Lemma 9.3: The space $Y$ of bounded (w.r.t. Euclidean norm) functions $g: \mathbb{R}^{d} \rightarrow \mathbb{C}^{9}$ is a complex normed space w.r.t. the norm $\sup _{\phi \in \mathbb{R}^{d}}|g(\phi)|$ and obviously $\Phi_{n, \lambda}$ is a sequence in $Y$. Because $\mathbb{C}^{9}$ is complete, so is $Y$ (see, for example, [46], Sec. 7.1). Thus to show that $\Phi_{n, \lambda}$ converges uniformly on $\mathbb{R}^{d}$ it suffices to show that it is a Cauchy sequence in $Y$; i.e., for all positive $\delta$ there is a positive integer $m$ such that for all integers with $j, k \geq m$ we have

$$
\sup _{\phi \in \mathbb{R}^{d}}\left|\Phi_{j, \lambda}(\phi)-\Phi_{k, \lambda}(\phi)\right|<\delta .
$$

We also observe that

$$
\sup _{\phi \in \mathbb{R}^{d}}\left|\Phi_{j, \lambda}(\phi)-\Phi_{k, \lambda}(\phi)\right|=\sup _{\phi \in D^{\prime}}\left|\Phi_{j, \lambda}(\phi)-\Phi_{k, \lambda}(\phi)\right|,
$$

which follows from the continuity of $\left|\Phi_{j, \lambda}(\phi)-\Phi_{k, \lambda}(\phi)\right|$ 
in $\phi$ and from $D^{\prime}$ being dense in $\mathbb{R}^{d}$. Equation (9.30) implies that (9.29) is equivalent to the statement

$$
\sup _{\phi \in D^{\prime}}\left|\Phi_{j, \lambda}(\phi)-\Phi_{k, \lambda}(\phi)\right|<\delta
$$

Clearly (9.31) holds because, by assumption, $\Phi_{n, \lambda}(\phi)$ converges uniformly on $D^{\prime}$. Thus $\Phi_{n, \lambda}$ converges uni- formly on $\mathbb{R}^{d}$, so that $a(\Phi(\cdot ; \phi), \lambda)$ exists for all $\phi$ in $\mathbb{R}^{d}$ and is continuous in $\phi$.

To complete the proof of Theorem 9.2 we now show that the conditions of Lemma 9.3 are fulfilled, i.e., that $\Phi_{n, \lambda}$ converges uniformly on $D^{\prime}$, for every $\lambda$. Clearly we have

$$
a\left(\Phi\left(\cdot ; \phi_{0}+2 \pi N \omega\right), \lambda\right)=\lim _{n \rightarrow \infty} \Phi_{n, \lambda}\left(\phi_{0}+2 \pi N \omega\right)=\exp (i \lambda 2 \pi N) a\left(\Phi\left(\cdot ; \phi_{0}\right), \lambda\right) \Phi^{T}\left(2 \pi N ; \phi_{0}\right),
$$

and to show that the limit in (9.32) is uniform on $D^{\prime}$ we estimate

$$
\begin{aligned}
& \left|\Phi_{n, \lambda}\left(\phi_{0}+2 \pi N \omega+2 \pi M\right)-\exp (i \lambda 2 \pi N) a\left(\Phi\left(\cdot ; \phi_{0}\right), \lambda\right) \Phi^{T}\left(2 \pi N ; \phi_{0}\right)\right| \\
& \quad=\left|\Phi_{n, \lambda}\left(\phi_{0}+2 \pi N \omega\right)-\exp (i \lambda 2 \pi N) a\left(\Phi\left(\cdot ; \phi_{0}\right), \lambda\right) \Phi^{T}\left(2 \pi N ; \phi_{0}\right)\right| \\
& \quad=\left|\frac{1}{n} \int_{2 \pi N}^{2 \pi N+n} \Phi\left(\theta ; \phi_{0}\right) \Phi^{T}\left(2 \pi N ; \phi_{0}\right) \exp (-i \lambda(\theta-2 \pi N)) d \theta-\exp (i \lambda 2 \pi N) a\left(\Phi\left(\cdot ; \phi_{0}\right), \lambda\right) \Phi^{T}\left(2 \pi N ; \phi_{0}\right)\right| \\
& \quad \leq \sqrt{3}\left|\frac{1}{n} \int_{2 \pi N}^{2 \pi N+n} \Phi\left(\theta ; \phi_{0}\right) \exp (-i \lambda \theta) d \theta-a\left(\Phi\left(\cdot ; \phi_{0}\right), \lambda\right)\right| .
\end{aligned}
$$

By using the first equality of (9.32) with $N=0$ and by noting that $\Phi\left(\cdot ; \phi_{0}\right)$ is bounded we have, for all $N$,

$$
\begin{aligned}
\lim _{n \rightarrow \infty} & \mid \frac{1}{n} \int_{2 \pi N}^{2 \pi N+n} \Phi\left(\theta ; \phi_{0}\right) \exp (-i \lambda \theta) d \theta \\
& -a\left(\Phi\left(\cdot ; \phi_{0}\right), \lambda\right) \mid \\
& =0
\end{aligned}
$$

Moreover, because $\Phi\left(\cdot ; \phi_{0}\right) \exp (-i \lambda \cdot)$ is almost periodic it follows (see [44], Chap. 3) that the convergence in (9.34) is uniform on the domain $\mathbb{Z}$ of $N$. Hence (9.33) implies that $\Phi_{n, \lambda}$ converges uniformly on $D^{\prime}$.

Thus we have proved, under the conditions of Theorem 9.2, that $\Lambda\left(\Phi\left(\cdot ; \phi_{0}\right)\right)$ is independent of $\phi_{0}$.

\section{Remark}

(3) Remark 2 shows that under the conditions of Theorem 9.1 the spin tune can indeed be discovered from a spectral analysis of the spin flow for arbitrary $\phi_{0}$. In particular, since the spin motion is quasiperiodic and the torus is well-tuned, the spectrum has at most countably many elements and $\Lambda\left(\Phi\left(\cdot ; \phi_{0}\right)\right)$ will consist of sharp "lines" which can then be measured. Moreover, off orbital resonance and under the conditions of Theorem 9.2, all $\Lambda\left(\Phi\left(\cdot ; \phi_{0}\right)\right)$ are equal. However, if the torus is ill-tuned but the spin motion is quasiperiodic [so that the spectrum $\Lambda\left(\Phi\left(\cdot ; \phi_{0}\right)\right)$ will consist of sharp lines], we expect that the union of the spectra $\Lambda\left(\Phi\left(\cdot ; \phi_{0}\right)\right)$ over the torus will contain uncountably many elements. The models in Remarks 13 and 14 in Sec. VI provide examples. Note that, in the absence of quasiperiodicity or even almost-periodicity, as, for example, in the Moser-Siegel model of Sec. VIII, there may be difficulties in even computing the spectrum. In practice, the spectrum can be obtained by tracking three mutually orthogonal spins along an orbit and storing their values at each of a very large number of turns before applying a discrete Fourier transform to the data (a well-known way of doing numerical Fourier analysis can also be found in [53]). But since this spectrum can be very dense, it can be difficult to identify the spin tunes. Thus it would be useful to begin with small amplitudes, i.e., tori with small $J$, and look for a $\omega_{s}$ close to that for the closed orbit, which can be calculated as in Sec. III. The spin tune at higher amplitudes could then be identified by continuing away from the closed orbit. We return to this theme in Sec. X. Finally, tracking a spin trajectory which is parallel to an ISF would give a spectrum without $\omega_{s}$.

\section{DISCUSSION AND CONCLUSION}

In the foregoing sections we have presented a thorough step by step account of the circumstances under which spin motion may be quasiperiodic on integrable particle orbits and have thereby put previous studies of the concept of spin tune onto a rigorous basis. In particular, we considered integrable orbits in $\mathcal{Q}\left(1, \omega_{1}, \ldots, \omega_{d} ; d+1\right)$ and by introducing certain conditions (e.g., Diophantine conditions) and assuming the existence of an ISF we obtained conditions under which the spin motion is in $\mathcal{Q}\left(1, \omega_{1}, \ldots, \omega_{d}, \omega_{s} ; d+2\right)$, where $\omega_{s}$ is a spin tune. We have also shown how, by introducing UPFs, the spin motion can be represented in terms of generalized Floquet forms.

The scenarios covered by our treatment and the relationships between them are summarized by the Venn diagram of Fig. 1.

The meanings of the domains in Fig. 1 are as follows.

Inside the black circle: all tori, i.e., for arbitrary real skew-symmetric $3 \times 3$ matrices $\mathcal{A}\left(\cdot, \cdot, J_{0}\right)$ and arbitrary orbital tunes $\omega\left(J_{0}\right)$, which are smooth and $2 \pi$-periodic in $\theta$ and $\phi$.

Inside the red ellipse: tori which have an ISF. 


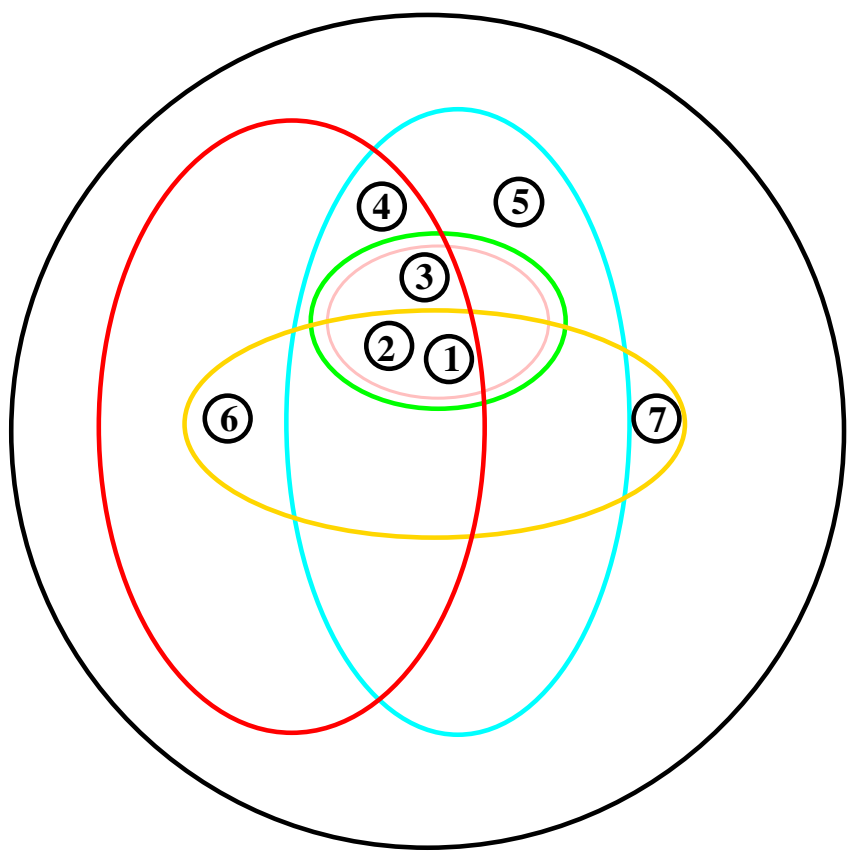

FIG. 1. (Color) The logical connections between the various scenarios.

Inside the blue ellipse: tori, which at every $\phi_{0}$ have a proper UPF (note that for those tori every spin trajectory is quasiperiodic).

Inside the green ellipse: tori which are well-tuned (see Sec. V).

Inside the yellow ellipse: tori which are off orbital resonance.

Inside the pink ellipse: tori with a uniform IFF.

The numbered circles label specific examples, as follows.

Example 1: the single resonance model off orbital resonance (see Sec. VII).

Example 2: the torus $J$ defined in Remark 13 in Sec. VI, for the case of irrational $\omega(J)$.

Example 3: the single resonance model on orbital resonance (see Sec. VII).

Example 4: the torus defined in Remark 13 in Sec. VI, for the case $\omega=1$.

Example 5: a torus defined in Remark 14 in Sec. VI.

Example 6: the Moser-Siegel model (for certain choices of the parameters $\omega, \sigma_{1}, \sigma_{2}$ ) (see Sec. VIII).

Example 7: see below.

Analytical solutions for spin motion or the ISF for the $\mathcal{A}$ 's arising in real storage rings cannot be obtained and it is not even known whether an ISF exists in general. Nevertheless, it seems that it usually exists for storage rings of interest. This is supported by a large amount of numerical work in which, for the cases studied, it was possible to construct at least a very good approximation to an ISF $[6,8,9,11-13,23,25]$. Note that in these simulations hard edge and some thin lens representations of fields were used so that the $\mathcal{A}$ 's were not smooth; see Remark 16 in Sec. VI.

Those studies also included calculations of the spin tune using SPRINT, either beginning with the pseudo- $u^{1}, u^{2}$-axes or using the SODOM2 algorithm whereby the spin tune is obtained by solving an eigenproblem for Fourier coefficients in an SU(2) formalism. Numerical simulations with both methods suggest that a spin tune normally exists if the torus is off orbital resonance. This is the situation considered by Theorem $6.5(\mathrm{~d})$ which implies that if we have an ISF $S$ and if a $2 \pi$-periodic unit vector exists which is nowhere parallel to $S$, then we almost always have a spin tune (see Remark 5 in Sec. VI). For this and the other results in this paper it has been convenient to prescribe that the $\mathcal{A}$ 's are smooth. But the demonstration of ISFs and spin tunes in $[6,8,9,11-13,23,25]$ indicates that the smoothness condition can often be relaxed. For convenience, in the remainder of this section we will use the term ISF in this spirit. We hope in the future to be able to present a treatment of the ISF and the spin tune in which the requirement of smoothness is relaxed.

An example of a situation where an ISF might not exist even in crude approximation, namely near $803.5 \mathrm{GeV}$ in the HERA ring, is given in [13]. If the spin motion is nonquasiperiodic, this case would correspond to Example 7 in Fig. 1.

The spin tune is a crucial quantity for characterizing the stability of spin motion. Spins behave somewhat like driven oscillators where the driver is the magnetic and electric fields along particle orbits. Near spin-orbit resonance, there is potential for marked qualitative changes in the spin motion which may then be quite erratic. The special nature of spin-orbit resonance is already clear from the fact that the ISF need not be unique at resonance; see Theorem 6.4. Moreover, it is clear that it would make little sense to define a spin-orbit resonance condition in terms of a UPR $\nu_{s}$ depending on $\phi_{0}$ : such a $\nu_{s}$ would in general take different values for different particles on a torus so that it would be impossible for the particles on a torus to be simultaneously on resonance and there would be no enhancement of our ability to systematize spin motion. This was the reason for insisting that a torus be well-tuned before considering spin-orbit resonance.

As mentioned in the Introduction and in Remark 5 of Sec. V, it is clear that $\omega_{s}$ can vary with the beam energy and with $J$. This is confirmed by simulations in SPRINT $[6,8,9,11-13,23,25]$, hence the name "amplitude dependent spin tune." To study the dependence of $\Xi_{J}$ on $J$ (recall Remark 5 of Sec. V), it is necessary to choose a "preferred" member of $\Xi_{J}$. For the calculations with SPRINT the choice is made as follows.

The spin tune $\nu_{0} \in[0,1)$ and the corresponding UPF are found on the closed orbit using the method outlined in Sec. III. Normally $\nu_{0}$ is set significantly different from zero in order to ensure that the direction of $\mathbf{n}_{0}$ is close to 
the "design" direction in spite of the presence of the usual misalignments of the ring [7,15,39]. Then as in Remark 3 in Sec. IX, the preferred spin tune at nonzero amplitudes is selected by requiring that it and the corresponding UPF vary continuously with amplitude and reduce continuously to the spin tune and UPF on the closed orbit.

With this procedure in place, simulations in which the ISF and the spin tune are calculated over a range of fixed amplitudes or energies indeed show that the spin motion can become erratic near a spin-orbit resonance. Moreover, in such cases there is a tendency for the ISF to become very sensitive to the parameter being varied. With this prescription, one also finds that the strongest variations of the ISF occur near low order resonances and that high order resonance effects are usually unimportant. For the details of these calculations and results, the reader is referred to $[6,8,9,11-13,23,25]$. These findings are consistent with perturbation theoretical calculations of the ISF as in [10]. The nonperturbative calculations also show that near spin-orbit resonance the spin tune tends to avoid exact fulfillment of the resonance condition. As we have already indicated in Remark 3 of Sec. VII, the spin tune for the single resonance model [see (7.11)] also avoids the spin-orbit resonance condition as $\sigma_{1}$ is varied through the condition $\sigma_{1}=\omega$.

In a ring without so-called Siberian snakes (see below) the closed orbit spin tune $\nu_{0}$ usually varies with the beam energy [7,14]. It is then sometimes implied in the literature that in a beam with a large energy spread, the synchrotron motion causes the particles to oscillate to and fro across spin-orbit resonances as the spin tune $\omega_{s}$ oscillates. This crossing of resonances is then supposed to be the source of the low beam polarization that would be seen. However, we have seen that a spin tune $\omega_{s}$ on a torus $\left(J_{1}, J_{2}, J_{3}\right)$ is a constant. As usual we assign $J_{3}$ to synchrotron motion. Thus $\omega_{s}$ does not oscillate and there is no resonance crossing. Nevertheless, we do expect that a large energy spread can lead to small beam polarization. This is explained by the fact that for particles of large enough $J_{3}$, $\mathbf{n}$ varies strongly with the $\phi_{3}$. Then the maximum permissible equilibrium beam polarization can indeed be small [7].

Studies of the effect on polarization of real resonance crossing can be found in [11-13,23]. The consequences for the polarization of crossing first order resonances are usually quantified using the Froissart-Stora formula [54]. But in $[11-13,23]$ it is shown that the Froissart-Stora approach can be generalized to describe the change of the polarization at the crossing of higher order resonances, too. This is a further illustration of the value of using a wisely defined spin tune for identifying resonances and for understanding their properties.

The spin tune can be obtained in SPRINT via the pseudo- $u^{1}, u^{2}$-axes or by using the SODOM2 algorithm. However, as we have seen in Sec. IX the spin tune might also be obtained (measured) by a spectral analysis of the spin motion. This offers an attractive alternative. As in the case of the other two methods the preferred spin tune would have to be identified among the many spectral lines by matching onto the spin tune of the closed orbit. We have also seen in Sec. IX that the ISF might be obtainable, if it exists, by spectral analysis of spin motion. Spectral analysis may thus lead to a criterion for deciding whether an invariant spin field exists. Other criteria for the existence of the ISF are already available for stroboscopic averaging and the SODOM2 algorithm.

We now complete this discussion by mentioning other quantities that have been used in attempts to define spin precession frequencies.

As explained in Remark 3 in Sec. III, for motion on the closed orbit the spin tune can be obtained trivially from the complex eigenvalues of the one-turn principal solution matrix. This does not normally work off the closed orbit since $\Phi\left(2 \pi ; \phi_{0}\right)=U(2 \pi) \exp \left(J 2 \pi \nu_{s}(U)\right) U^{T}(0)$ and for this the $\exp \left( \pm 2 \pi i \nu_{s}(U)\right)$ are generally not the complex eigenvalues. So the reader will agree that on synchrobetatron orbits the spin tune usually cannot be obtained from the complex eigenvalues of the one-turn principal solution matrix. In fact, in general the real eigenvector of the general one-turn principal solution matrix $\Phi\left(\theta+2 \pi ; \phi_{0}\right) \Phi^{T}\left(\theta ; \phi_{0}\right)$ starting at $\theta$ is not even a spin trajectory and is not parallel to an ISF ([55], p. 27, for example); the term "spin closed orbit," which is sometimes used for the ISF, is inappropriate. Moreover in the simulations with SPRINT the sensitivity of the ISF to variation of parameters shows no correlation with the spin precession rate extracted from the complex eigenvalues of the one-turn principal solution matrix. Of course eigenvalues of one-turn principal solution matrices are easy to calculate but for $J \neq 0$ they usually have no useful function.

The model described in Eq. 21 of [32] provides an example. It concerns orbits which are said in [31] to be exceptional. It is stated there that exceptional orbits are characterized by the feature that the spin tune depends on orbital phase and this is discussed in close conjunction with Stern-Gerlach forces. As we point out in the Introduction, Stern-Gerlach forces can have no practical relevance for understanding spin resonance. The model in [32] involves the single resonance model (see Sec. VII) and a single thin lens Siberian snake. A Siberian snake $[56,57]$ of the kind used here is a magnet system that rotates a spin by the angle $\pi$ around an axis in the plane of the ring ([55], p. 71). In the notation of Sec. VII the parameters in [32] are $\sigma_{1}=\omega$ and $\sigma_{2} \sqrt{2 J}=3 / 2$. At all $\phi_{0}$ the one-turn principal solution matrix $\Phi\left(2 \pi ; \phi_{0}\right)$ starting at the snake represents a rotation around the vertical by an angle depending linearly on $\phi_{0}$. Then the ISF is vertical at the snake at all $\phi_{0}$ on this torus and for this zero-measure range of $J$, the ISF at the snake is the 
same as the real unit-length eigenvector of the one-turn principal solution matrix. The orbital tune $\omega$ is arbitrary. The eigentunes extracted from the complex eigenvalues of $\Phi\left(2 \pi ; \phi_{0}\right)$ vary linearly with $\phi_{0}$. Therefore if a particle is followed along an orbit, these eigentunes normally change abruptly between one turn and the next. Other one-turn eigentunes would be obtained if other positions around the ring were chosen for $\theta=0$. Nevertheless, it is claimed in [31,32] that the eigentune for $\Phi\left(2 \pi ; \phi_{0}\right)$ is $2 \pi \times$ spin tune. Obviously this is not a spin tune in the sense of our treatment. We have taken account of the fact that an eigentune in the $\mathrm{SU}(2)$ formalism used in [31,32] is one half of a corresponding eigentune in our formalism. Although this $\Phi\left(2 \pi ; \phi_{0}\right)$ and its eigentune are discussed in association with S-G forces, the inclusion of S-G forces is not necessary to obtain either. In fact, by exploiting techniques additional to those used in this paper one can show that these parameters simply provide an example of ill-tuning and that this case would be entered next to Example 6 in the diagram of Fig. 1. It is not clear from $[31,32]$ whether these phase dependent eigentunes serve some useful function such as indicating the stability of spin motion. We give another example of the use of the eigentune of a one-turn principal solution matrix below.

The situation with regard to the utility of eigenvectors and eigentunes is more subtle for tori on orbital resonance where $\omega_{1}, \omega_{2}, \ldots$ are rational. The orbit and $A$ are then periodic over an appropriate number $M$ of whole turns. Thus in analogy with the method in Remark 6 in Sec. III, there is a possibility of calculating $\mathbf{n}$ at $\theta=0$ as the unitlength real eigenvector of the $M$-turn principal solution matrix and in general it would be a function of $\phi_{0}$. The imaginary part of the exponent of a complex eigenvalue of this principal solution matrix would provide the $M$-turn advance of the phase of spin rotation around $\mathbf{n}$ and this could be used to obtain the average one-turn spin phase advance. This would usually depend on $\phi_{0}$ and in such cases it could not be used to define a spin tune, but it could be used to define a $\nu_{\mathcal{V}}$ [see (6.26)].

So, as is usual at orbital resonance, an ISF can exist in general but normally there is no spin tune. We note in passing that the $M$-turn complex eigentunes are $2 \pi$-periodic in $\theta$ and $\phi$, just like $\mathcal{A}$. Moreover, because eigenvalues of matrices are invariant under similarity transformations, the eigentunes are invariant when the starting point for the eigenanalysis is shifted along the orbit. Examples of an unwise use of the term spin tune can be found in [58] where the dependence of the multiturn eigentune on $\phi_{0}$ is made explicit; see also [31,32]. Again, a "tune" depending on $\phi_{0}$ cannot be used for studying spin-orbit resonance. Calling such a quantity a spin tune can create confusion.

If, on orbital resonance with rational tunes, the torus is ill-tuned, then one can expect that either there are more or fewer proper UPRs than one would have on a well-tuned torus. Thus a spectral analysis along the lines of that in Sec. IX, applied to every $\phi_{0}$ on the torus, could be a useful diagnostic tool to signal these two cases of illtuning. Note that in the examples in Remarks 13 and 14 in Sec. VI there are too many UPRs. Therefore one might expect that on orbital resonance with rational tunes an illtuned torus had too many proper UPRs.

Although an $M$-turn eigentune is in general $\phi_{0}$ dependent, we might expect that an approximation to the spin tune $\omega_{s}$ on a well-tuned torus off orbital resonance could be obtained by setting the orbital tunes to rational values near the actual tunes but such that $M$ were very large. Indeed this is the essence of a popular perception. In effect, although usually not clearly stated, the underlying hope is that, for the smooth guide fields of real rings and for large enough $M$, the average one-turn spin phase advance is only very weakly dependent on $\phi_{0}$ and that therefore a good approximation to $\omega_{s}$ can be obtained. That would be consistent with the heuristic expectation that for large enough $M$ the influence of the initial $\phi_{0}$ become diluted. Such behavior might also be expected if the high order Fourier coefficients in the $\tilde{c} \mathcal{V}(\theta, \phi-\omega \theta)$ in (6.21) are very small owing to the smoothness of the fields. See [24], p. 66, for a hint of how the Fourier coefficients come in here. We hope in the future to be able to present a rigorous treatment of this approximation. This approach has been adopted in [58] to indicate that off orbital resonance the spin tune is a half integer at most $J$ in the model to be discussed in the next paragraph. However, any attempt to find an approximate value for a spin tune by using $M$-turn maps for nearby rational tunes should at least be checked for convergence and consistency.

Further discussion around the topic of rational tunes can be found in [51] in which the nature of the so-called "snake resonances" is studied. These refer to a large loss of beam polarization during acceleration in models in which the spin motion in most of the ring is approximated by the single resonance model and the spin motion is stabilized by pairs of idealized, i.e., thin lens, Siberian snakes [59]. The Siberian snakes have the effect of fixing $\nu_{0}$ at $1 / 2$ independently of the beam energy ([55], p. 70). Snake "resonances" occur at rational orbital tunes $\omega$ for which, in the notation of (5.10), $1 / 2=m_{0}+m_{1} \omega$ with odd $m_{1}$. In [51] it is made evident that at these tunes and for most $J \neq 0$ there is no amplitude dependent spin tune so that one is not dealing with spin-orbit resonances. Moreover, at most orbital amplitudes the ISF defined there (i.e., without insistence on smoothness) is irreducibly discontinuous at some orbital phases [51]. Of course, since rational tunes correspond to orbital resonance, it should come as no surprise, given the content of our paper, that there is no amplitude dependent spin tune in this case. We have explicitly chosen our nomenclature to be consistent with earlier usage [18] and thereby contribute clarity to the classification of phenomena. It could be 
that the pathological behavior in the models, namely the large loss of polarization, is due to the use of the simplified but singular representation for the snake fields. In this connection we note with interest that according to simulations for RHIC, the loss of polarization during acceleration is less severe when the simulations are carried out with the magnetic fields of real snakes than with the singular fields of thin lens snakes [60]. This indicates that predictions from simplified, mathematically singular models should be treated with some caution. In any case the use of the term "snake resonance" is a good illustration of the confusion that arises from an imprecise use of the concept of spin-orbit resonance.

A model involving the single resonance model and two thin snakes provides the second example of the use of the eigentune from the one-turn principal solution matrix. Simulations described in [59] (pp. 98-100) show some loss of polarization for all nonzero $2 \sigma_{2}^{2} J$ during acceleration, even away from the rational orbital tunes associated with snake "resonances." It is implied there that this loss stems from the fact that during the acceleration the eigentune from the one-turn principal solution matrix, which in [59] is called the "perturbed spin tune," oscillates to and fro across a spin-orbit resonance as the orbital phase advances from turn to turn. But in this example the one-turn eigenvector of the principal solution matrix is usually not even parallel to the ISF. So it is even more difficult to imagine that the perturbed spin tune can characterize long term spin motion. Thus an alternative picture for the loss of polarization must be sought.

As mentioned in Remarks 1 and 4 in Sec. VII, for the straightforward single resonance model a spin tune does exist on orbital resonance.

This completes our discussion of notions of spin precession frequency.

We now conclude by summarizing the main message. This is, that, by just employing the Lorentz force and the T-BMT equation and with the help of the concept of quasiperiodicity, we are able to provide a rigorous and broad treatment of the concepts of proper uniform precession rate, spin tune, and the invariant spin field by common methods used for ordinary differential equations. This allows us to focus on the main phenomena without the distraction of perturbations such as noise, collective effects, and synchrotron radiation. In principle they can be included by using perturbation theory. We have discussed putative Stern-Gerlach forces in the Introduction. The advantages of a clear, universally accepted and useful definition of spin tune have been made evident by the examples in the foregoing paragraphs.

\section{ACKNOWLEDGMENTS}

We thank G. H. Hoffstaetter, H. Mais, G. Ripken, M. Vogt, and K. Yokoya for important and fruitful discussions on this and related topics. In addition, J. A. E. grate- fully acknowledges support from DOE Grant No. DEFG03-99ER41104 and from DESY during a sabbatical in 1997-1998 when the core elements of this work were first assembled into a manuscript.

Note added in proof.-Our discussion in Sec. X of "snake resonances" concentrated on some aspects of the theory. For recent experimental work around "snake resonance" vertical orbital tunes, see [61].

[1] J. D. Jackson, Classical Electrodynamics (Wiley, New York, 1998), 3rd ed.

[2] D. P. Barber, K. Heinemann, and G. Ripken, Z. Phys. C 64, 117 (1994).

[3] J. A. Ellison and K. Heinemann, "Periodic Spin Fields and Phase Space Densities: Stroboscopic Averaging and the Ergodic Theorem" (to be published).

[4] K. Gottfried, Quantum Mechanics: Fundamentals (Addison-Wesley, Reading, MA, 1989).

[5] K. Heinemann and D. P. Barber, Nucl. Instrum. Methods Phys. Res., Sect. A 463, 62 (2001); 469, 294 (2001).

[6] K. Heinemann and G. H. Hoffstaetter, Phys. Rev. E 54, 4240 (1996).

[7] D. P. Barber et al., in Proceedings of ICFA Workshop "Quantum Aspects of Beam Physics," Monterey, CA, 1998, edited by P. Chen (World Scientific, Singapore, 1999).

[8] D. P. Barber, G. H. Hoffstaetter, and M. Vogt, in Proceedings of the 1998 European Particle Accelerator Conference (EPAC98), Stockholm, 1998 (IOP, London, 1998), also available at http://epac.web.cern.ch/EPAC/ Welcome.html.

[9] D. P. Barber, G. H. Hoffstaetter, and M. Vogt, in Proceedings of the 13th International Symposium on High Energy Spin Physics, Protvino, Russia, 1998 (World Scientific, Singapore, 1999).

[10] S. R. Mane, Phys. Rev. A 36, 105 (1987).

[11] G. H. Hoffstaetter and M. Vogt, Phys. Rev. E 70, 056501 (2004).

[12] G. H. Hoffstaetter, "A modern view of high energy polarized proton beams," Springer Tracts in Modern Physics (to be published).

[13] M. Vogt, Ph.D. thesis, University of Hamburg [DESY Technical Report No. DESY-THESIS-2000-054, 2000].

[14] A.W. Chao, Nucl. Instrum. Methods 180, 29 (1981). Modern notation: replace $\mathbf{n}$ by $\mathbf{n}_{0}$.

[15] D. P. Barber and G. Ripken, in Handbook of Accelerator Physics and Engineering, edited by A.W. Chao and M. Tigner (World Scientific, Singapore, 2002), 2nd ed.

[16] V.V. Balandin and N. I. Golubeva, DESY Technical Report No. 98-16, 1998.

[17] Yu. Eidelman and V. Yakimenko, Part. Accel. 50, 261 (1995).

[18] K. Yokoya, DESY Technical Report No. 99-006, 1999; physics/9902068. 
[19] S. R. Mane, Nucl. Instrum. Methods Phys. Res., Sect. A 498, 1 (2003).

[20] Ya. S. Derbenev and A. M. Kondratenko, Sov. Phys. JETP 35, 230 (1972).

[21] Ya. S. Derbenev and A. M. Kondratenko, Sov. Phys. JETP 37, 968 (1973).

[22] K. Yokoya, DESY Technical Report No. 86-57, 1986.

[23] D. P. Barber, G. H. Hoffstaetter and M. Vogt, in SPIN 2000: 14th International Spin Physics Symposium, AIP Conf. Proc. No. 570 (AIP, New York, 2001).

[24] D. P. Barber, K. Heinemann and G. Ripken, DESY Technical Report No. M-92-04, 1999, second revised version..

[25] G. H. Hoffstaetter, M. Vogt, and D. P. Barber, Phys. Rev. ST Accel. Beams 2, 114001 (1999).

[26] Dynamical Systems II, Encyclopedia of Mathematical Sciences V, edited by Y. G. Sinai (Springer, New York, 1989).

[27] I. P. Cornfeld, S.V. Fomin, and Y. G. Sinai, Ergodic Theory (Springer, New York, 1982).

[28] K. Heinemann, DESY Technical Report No. 96-229, 1996; physics/9611001.

[29] A. A. Pomeransky, R. A. Senkov, and I. B. Khriplovich, Phys. Usp. 43, 1055 (2000); see also I. B. Khriplovich and A. A. Pomeransky, Surv. High Energy Phys. 14, 145 (1999); gr-qc/9809069.

[30] Ya.S. Derbenev, University of Michigan-Ann Arbor Technical Report No. UM-HE-90-30, 1990.

[31] S. R. Mane, Nucl. Instrum. Methods Phys. Res., Sect. A 498, 52 (2003).

[32] S. R. Mane, in SPIN 2002: 15th International Spin Physics Symposium and Workshop on Polarized Electron Sources and Polarimeters, edited by Y.I. Makdisi, A. U. Luccio, and W.W. MacKay, AIP Conf. Proc. No. 675 (AIP, New York, 2003).

[33] D. P. Barber, J. A. Ellison, and K. Heinemann, in SPIN 2000: 14th International Spin Physics Symposium (Ref. [23]).

[34] H. Amann, Ordinary Differential Equations: Introduction to Nonlinear Analysis (de Gruyter, Berlin, 1990).

[35] J. K. Hale, Ordinary Differential Equations (Krieger, Malabar, FL, 1980), 2nd ed.

[36] Y. Aharonov and J. Anandan, Phys. Rev. Lett. 58, 1593 (1987).

[37] J. N. Franklin, Matrix Theory (Prentice-Hall, Englewood Cliffs, NJ, 1968).

[38] H. Goldstein, Classical Mechanics (Addison-Wesley, New York, 1982), 2nd ed.
[39] D. P. Barber et al., DESY Technical Report No. 85-44, 1985). Modern notation: replace $\mathbf{n}$ by $\mathbf{n}_{0}$.

[40] P. Lochak and C. Meunier, Multiphase Averaging for Classical Systems (Springer, New York, 1988).

[41] T.W. Koerner, Fourier Analysis (Cambridge University, Cambridge, 1988).

[42] W. Maak, Fastperiodische Funktionen (Springer, Berlin, 1967), 2nd ed.

[43] V. I. Arnold, Mathematische Methoden der klassischen Mechanik (Birkhaeuser, Basel, 1988).

[44] A. M. Fink, in Almost Periodic Differential Equations, Lecture Notes in Mathematics Vol. 377 (Springer, Berlin, 1974).

[45] H. S. Dumas, J. A. Ellison, and M. Vogt, SIAM J. Appl. Dynam. Syst. 3, 409 (2004).

[46] J. Dieudonne, Foundations of Modern Analysis (Academic, New York, 1960).

[47] S. Lang, Real Analysis (Addison-Wesley, Reading. MA, 1973).

[48] T. Yoshizawa, Stability Theory and the Existence of Periodic Solutions and Almost Periodic Solutions (Springer, New York, 1975).

[49] S. R. Mane, Nucl. Instrum. Methods Phys. Res., Sect. A 321, 21 (1992).

[50] I. S. Gradstein and I. M. Ryshik, Tables of Integrals, Series, and Products (Academic, New York, 1965).

[51] D. P. Barber et al., in SPIN 2002: 15th International Spin Physics Symposium and Workshop on Polarized Electron Sources and Polarimeters (Ref. [32]).

[52] C. L. Siegel and J. K. Moser, Lectures on Celestial Mechanics (Springer, New York, 1971).

[53] J. Laskar, Physica (Amsterdam) 67D, 257 (1993).

[54] M. Froissart and R. Stora, Nucl. Instrum. Methods 7, 297 (1960).

[55] B. Montague, Phys. Rep. 113, 1 (1984).

[56] Ya. S. Derbenev and A. Kondratenko, Sov. Phys. Dokl. 20, 562 (1976).

[57] Ya. S. Derbenev et al., Part. Accel. 8, 115 (1978).

[58] S. R. Mane, Nucl. Instrum. Methods Phys. Res., Sect. A 480, 328 (2002).

[59] S. Y. Lee, Spin Dynamics and Snakes in Synchrotrons (World Scientific, Singapore, 1997).

[60] M. Xiao and T. Katayama, University of Tokyo Technical Report No. CNS-REP-51, 2003.

[61] M. Bai et al., "Snake depolarizing resonance observed in RHIC," in SPIN 2004: 16th International Spin Physics Symposium, Trieste, Italy, 2004 (World Scientific, to be published). 